\title{
A One-Pot, Microwave-Influenced Synthesis of Diverse Small Molecules by Multicomponent Reaction Cascades
}

\author{
Soumava Santra and Peter R. Andreana* \\ Department of Chemistry, \\ Wayne State University, \\ 5101 Cass Ave, \\ Detroit, MI 48202
}

\section{Supporting Information}

Materials and Methods: All reagents were purchased from Aldrich unless otherwise mentioned. 4-Hydroxybenzylamine was purchased from Matrix Scientific. 4-Hydroxy3-methoxybenzylamine hydrochloride was purchased from Alfa Aesar. Except as otherwise indicated, reactions were carried out under argon. Microwave reactions were conducted in a capped vial using a CEM Discover System. All reactions were monitored using thin layer chromatography on $0.25 \mathrm{~mm}$ Dynamic Adsorbents, L.L.C. precoated silica gel (particle size 0.03-0.07 mm, catalog no. 84111, lot \# LA2006). Column chromatography was performed using Whatman Purasil $60 \AA$ (230-400 mesh ASTM) silica gel. Yields refer to chromatographically and spectroscopically pure compounds except where noted in the manuscript (Table 1). Diastereomeric ratios were determined from ${ }^{1} \mathrm{H}$ NMR spectra of unquenched reactions. Proton and carbon-13 NMR spectra were recorded on Varian Mercury 400, Varian Unity 500 and Varian 500 Direct Drive System spectrometers. The residual $\mathrm{CDCl}_{3}$ singlet at $\delta 7.26 \mathrm{ppm}$ and $\delta 77 \mathrm{ppm}$ were used as the standard for ${ }^{1} \mathrm{H}$ NMR and ${ }^{13} \mathrm{C}$ NMR spectra respectively. Mass spectra were recorded on a Micromass GCT at $70 \mathrm{eV}$.

General Procedure 1 (Starting with four separate substrates). In a $10 \mathrm{~mL}$ vial (CEM Discover System) equipped with a magnetic stir bar, benzylamine (15 mg, $0.14 \mathrm{mmol}$ ), 2,2-dimethylpropanal (12 mg, $0.14 \mathrm{mmol}$ ), trans-3-benzoylacrylic acid (25 mg, 0.14 mmol) and benzyl isocyanide ( $17 \mu \mathrm{L}, 0.14 \mathrm{mmol})$ were added to a mixture of $2.5 \mathrm{~mL}$ of distilled water ${ }^{\mathrm{a}}$. The vial was capped properly and placed in the microwave. The

(a) Other protic solvents used: (a) $\mathrm{MeOH}$, (b) Ethanol, (c) isopropylalcohol, and combinations thereof. All protic solvents used gave good yields of either compound Type A or Type B. Due to their relative ability to absorb microwave irradiation, water gave the best overall results in terms of $\%$ conversion and isolatable yield. 
microwave was then run at $300 \mathrm{~W}, 200{ }^{\circ} \mathrm{C}, 18 \mathrm{Bar}$ for $20-25 \mathrm{~min}$. After cooling the vial to room temperature, ethyl acetate was added and the mixture was shaken thoroughly. The organic layer was separated and the aqueous layer was extracted with ethyl acetate (2 x $2 \mathrm{~mL})$. The organic layers were combined and washed with saturated $\mathrm{NaHCO}_{3}(3 \times 3$ $\mathrm{mL}), 1 \mathrm{~N} \mathrm{HCl}(3 \times 3 \mathrm{~mL})$ which neutralized any remaining isocyanide, and brine $(3 \times 3$ $\mathrm{mL}$ ). The organic layer was separated. All aqueous layers were combined and extracted with $3 \mathrm{~mL}$ of ethyl acetate. The organic layers were combined, dried with $\mathrm{Na}_{2} \mathrm{SO}_{4}$ and filtered. The filtrate was concentrated under vacuum and the residue was purified by silica gel column chromatography using a 1:2 mixture of ethyl acetate and hexane as the eluent.

General Procedure 2 (Starting from a preformed acyclic Ugi product). Preformed, acyclic Ugi products could be obtained by simply combining the four substrates in some solvent $^{14}$. Please see General Procedure 3. To a $10 \mathrm{~mL}$ vial (CEM Discover System) equipped with a magnetic stirring bar, $50 \mathrm{mg}$ of the acyclic Ugi product was added along with $2.5 \mathrm{~mL}$ of distilled water. The vial was capped properly and placed in the microwave. Then the microwave was run for $20-25 \mathrm{~min}$ at $200{ }^{\circ} \mathrm{C}, 18 \mathrm{Bar}$ and $300 \mathrm{~W}$. After cooling the vial to room temperature, ethyl acetate was added and the mixture was shaken thoroughly. The organic layer was separated and the aqueous layer was extracted with ethyl acetate $(2 \times 2 \mathrm{~mL})$. The organic layers were combined, dried with $\mathrm{Na}_{2} \mathrm{SO}_{4}$ and filtered. The filtrate was concentrated under vacuum and the residue was purified by silica gel column chromatography using 1:2 mixture of ethyl acetate and hexane as the eluent.

General Procedure 3 (for acyclic intermediates): In a $10 \mathrm{~mL}$ round-bottomed flask, 4methoxybenzylamine $(0.3 \mathrm{~g}, 2.19 \mathrm{mmol})$ was dissolved in $3 \mathrm{~mL}$ of distilled water (or 3 $\mathrm{mL}$ of $\mathrm{MeOH})$ and 2,2-dimethylpropanal $(0.24 \mathrm{~mL}, 2.19 \mathrm{mmol})$ was added. The mixture was stirred for $10 \mathrm{~min}$. Then fumaric acid monoethyl ester $(0.32 \mathrm{~g}, 2.19 \mathrm{mmol})$ and benzyl isocyanide $(0.27 \mathrm{~mL}, 2.19 \mathrm{mmol})$ were added consecutively. The resulting solution was allowed to stir overnight at room temperature and then quenched with $1 \mathrm{~mL}$ 
of $1 \mathrm{~N} \mathrm{HCl}$. To the mixture, $3 \mathrm{~mL}$ of $\mathrm{CH}_{2} \mathrm{Cl}_{2}$ was added and the reaction was vigorously stirred. The organic layer was separated and the aqueous layer was extracted with $\mathrm{CH}_{2} \mathrm{Cl}_{2}(2 \times 2 \mathrm{~mL})$. The organic layers were collected, dried over $\mathrm{Na}_{2} \mathrm{SO}_{4}$ and filtered. The filtrate was concentrated under vacuum and the residue was purified by silica gel column chromatography using a 1:2 mixture of ethyl acetate and hexane as eluent.

Ethyl 2-(4-(4-methoxybenzyl)-5-tert-butyl-1-benzyl-3,6-dioxopiperazin-2-yl)acetate (5)

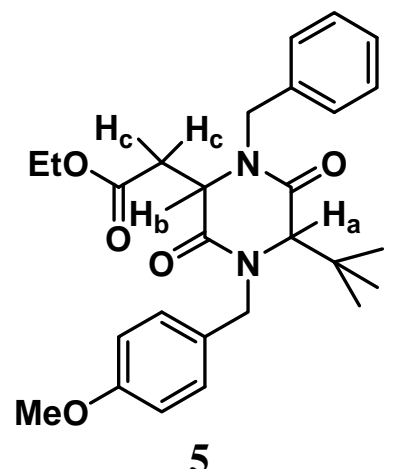

${ }^{1} \mathrm{H}$ NMR (500 MHz, $\left.\mathrm{CDCl}_{3}\right): \delta$ 7.18-7.38 (m, 7H, aryl), $6.88(\mathrm{~d}, 2 \mathrm{H}, J=8.6 \mathrm{~Hz}$, aryl), 5.6 $(\mathrm{d}, 1 \mathrm{H}, J=14.7 \mathrm{~Hz}, \mathrm{Bn}), 5.11(\mathrm{~d}, 1 \mathrm{H}, J=15.7 \mathrm{~Hz}, \mathrm{Bn}), 4.45(\mathrm{dd}, 1 \mathrm{H}, J=3.5,5.8 \mathrm{~Hz}$, $\left.\mathrm{H}_{\mathrm{b}}\right), 4.26(\mathrm{~d}, 1 \mathrm{H}, J=15.7 \mathrm{~Hz}, \mathrm{Bn}), 3.99-4.17\left(\mathrm{~m}, 2 \mathrm{H},-\mathrm{CH}_{2} \mathrm{CH}_{3}\right), 3.92(\mathrm{~d}, 1 \mathrm{H}, J=14.7$ $\mathrm{Hz}, \mathrm{Bn}), 3.81\left(\mathrm{~s}, 3 \mathrm{H}, \mathrm{OCH}_{3}\right), 3.78\left(\mathrm{~s}, 1 \mathrm{H}, \mathrm{H}_{\mathrm{a}}\right), 3.17\left(\mathrm{dd}, 1 \mathrm{H}, J=3.5,17.3 \mathrm{~Hz}, \mathrm{H}_{\mathrm{c}}\right), 2.95$ $\left(\mathrm{dd}, 1 \mathrm{H}, J=6.1,17.3 \mathrm{~Hz}, \mathrm{H}_{\mathrm{c}}\right), 1.26\left(\mathrm{t}, 3 \mathrm{H}, J=7.1 \mathrm{~Hz}, \mathrm{CH}_{3}\right), 1.1$ (s, 9H, t-butyl).

${ }^{13} \mathrm{C}$ NMR $\left(125 \mathrm{MHz}, \mathrm{CDCl}_{3}\right): \delta 169.9,167.7,166.7,166.7,159.2,136.5,129.5,128.8$, $127.7,127.5,114.3,67.6,60.9,55.6,55.2,50.8,46.8,40.0,34.8,28.4,14.1$.

HRMS: EIMS $\left(\mathrm{M}^{+}\right)$calcd for $\mathrm{C}_{27} \mathrm{H}_{34} \mathrm{~N}_{2} \mathrm{O}_{5} 466.2468$, found 466.2464. 
${ }^{1}$ H-NMR (Compound 5)

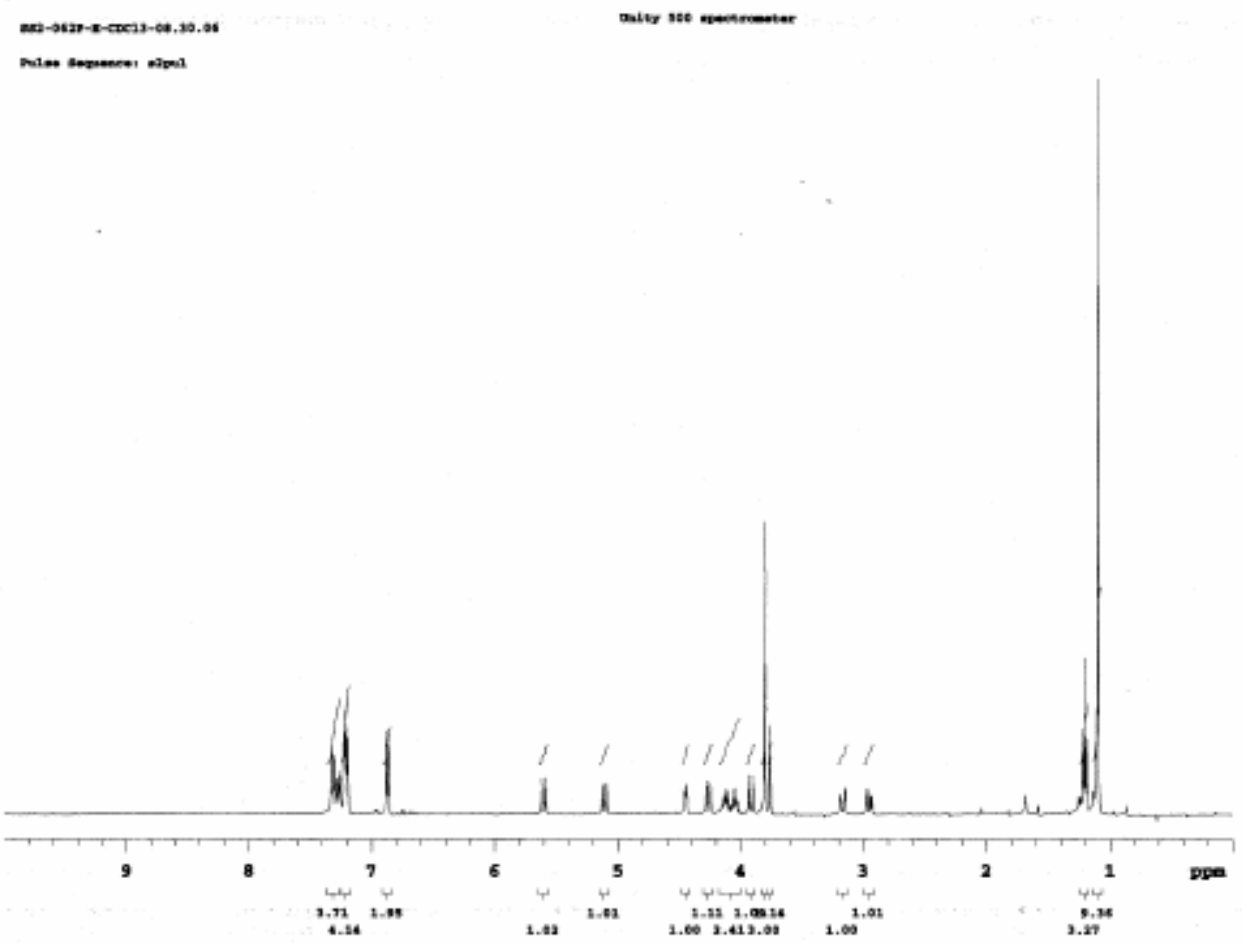

\section{${ }^{13}$ C-NMR (Compound 5)}

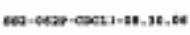

datty seo postimates.

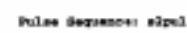

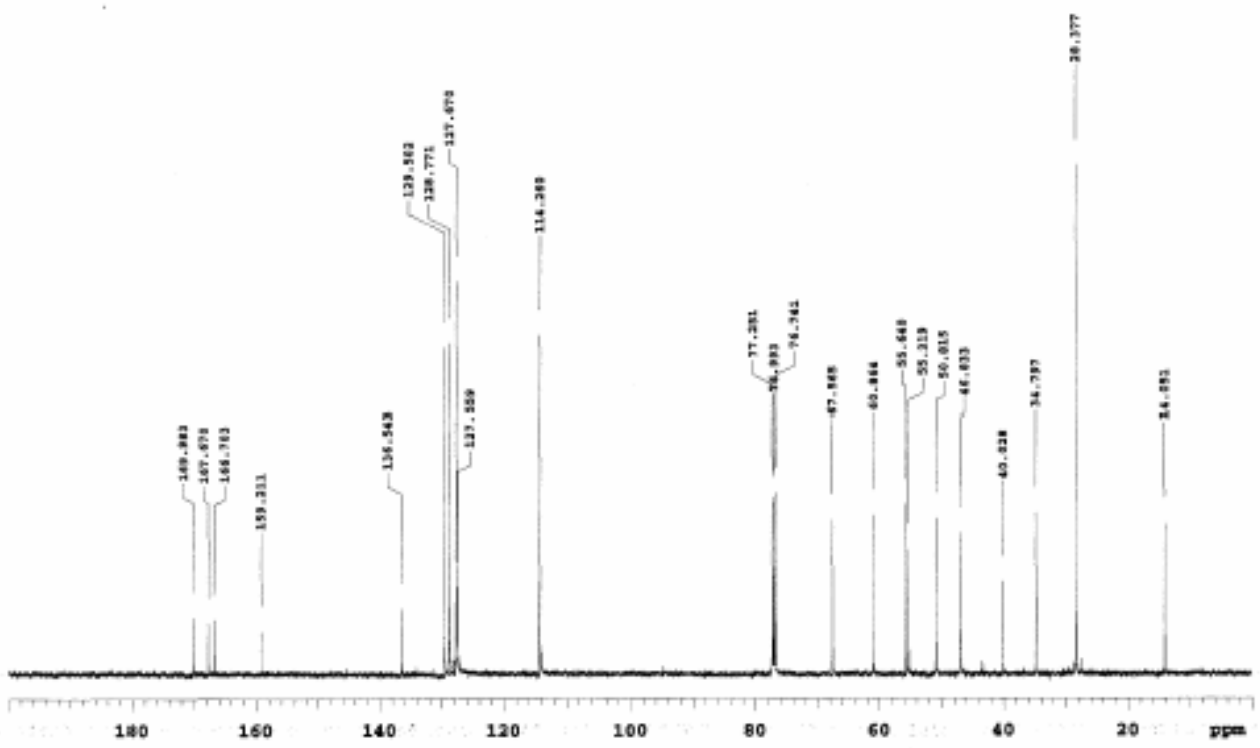


(E)-Ethyl 3-(N-(1-(benzylcarbamoyl)-2,2-dimethylpropyl)-N-(4-methoxybenzyl)carbamoylacrylate (6)<smiles>CCOC(=O)/C=C/C(=O)N(Cc1ccc(OC)cc1)C(C)(C(=O)NCc1ccccc1)C(C)(C)C</smiles>

${ }^{1} \mathrm{H}$ NMR (500 MHz, $\mathrm{CDCl}_{3}$ ): $\delta$ 7.1-7.35 (m, 6H, aryl), $6.96(\mathrm{~d}, 2 \mathrm{H}, J=8.1 \mathrm{~Hz}$, aryl), 6.75 $(\mathrm{d}, 2 \mathrm{H}, J=8.6 \mathrm{~Hz}$, aryl), $6.62(\mathrm{~d}, 1 \mathrm{H}, J=15.2 \mathrm{~Hz}, \mathrm{HC}=\mathrm{CH}), 5.27(\mathrm{~d}, 1 \mathrm{H}, J=17.2 \mathrm{~Hz}$, $\mathrm{Bn}$ ), 4.96-5.18 (br, 1H,), 4.72 (d, 1H, $J=17.2 \mathrm{~Hz}, \mathrm{Bn}), 4.35$ (dd, $1 \mathrm{H}, J=5.6,14.2 \mathrm{~Hz}$ ), 4.03-4.22 (m, 3H), $3.8\left(\mathrm{~s}, 3 \mathrm{H}, \mathrm{OCH}_{3}\right), 1.24\left(\mathrm{t}, 3 \mathrm{H}, J=7.1 \mathrm{~Hz}, \mathrm{CH}_{3}\right), 1.1$ (s, 9H, t-butyl).

${ }^{13} \mathrm{C}$ NMR $\left(125 \mathrm{MHz}, \mathrm{CDCl}_{3}\right): \delta 168.7,167.2,165.3,158.6,137.6,134.2,131.4,128.7$, $127.9,127.5,127.2,114.1,60.9,55.2,43.4,36.7,27.6,14.0$.

HRMS: EIMS $\left(\mathrm{M}^{+}\right)$calcd for $\mathrm{C}_{27} \mathrm{H}_{34} \mathrm{~N}_{2} \mathrm{O}_{5} 466.2468$, found 466.2481.

\section{${ }^{1}$ H-NMR (Compound 6)}

$$
\text { as2-032p }
$$
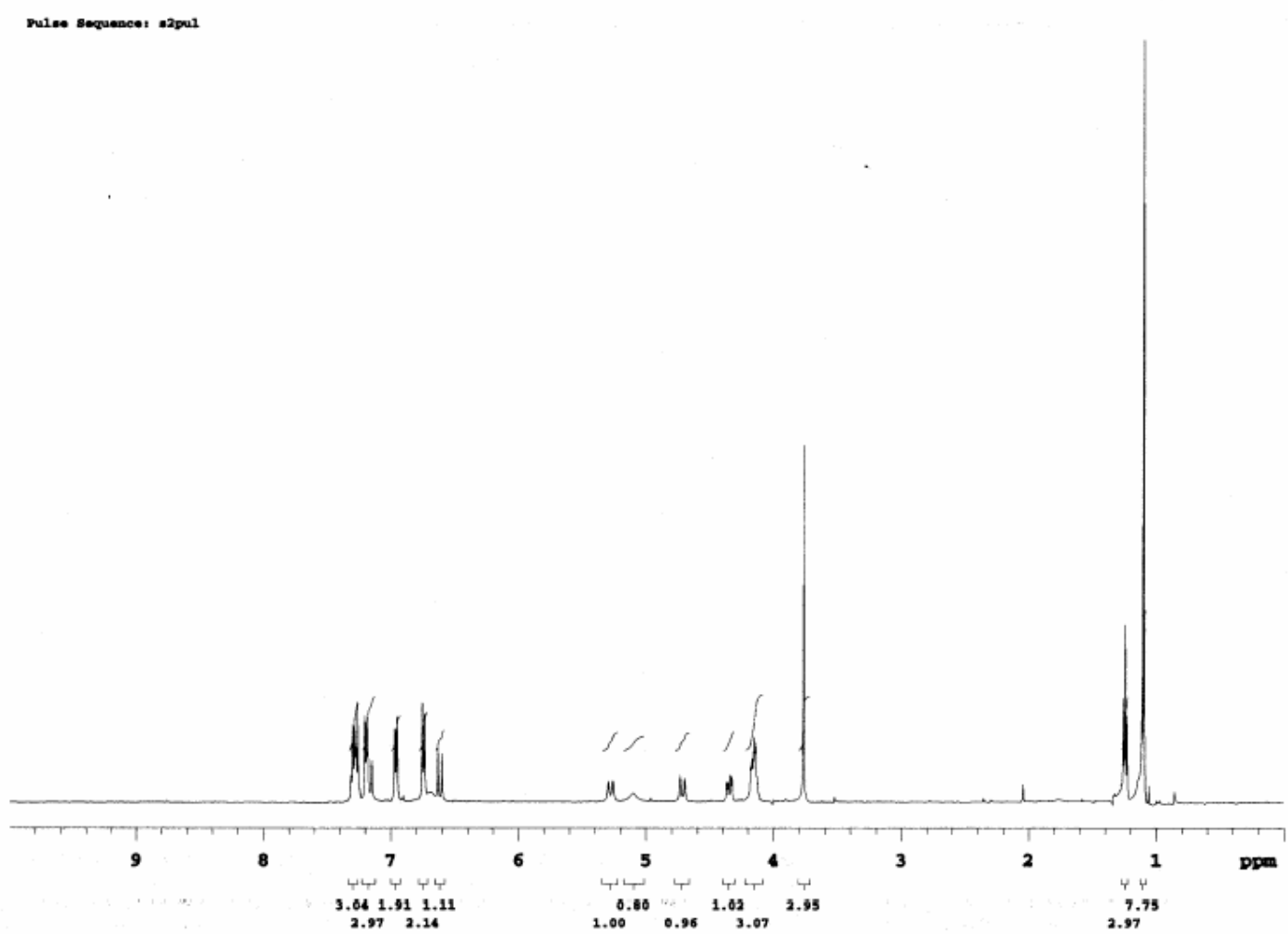


\section{${ }^{13}$ C-NMR (Compound 6)}

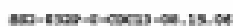

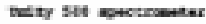

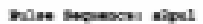

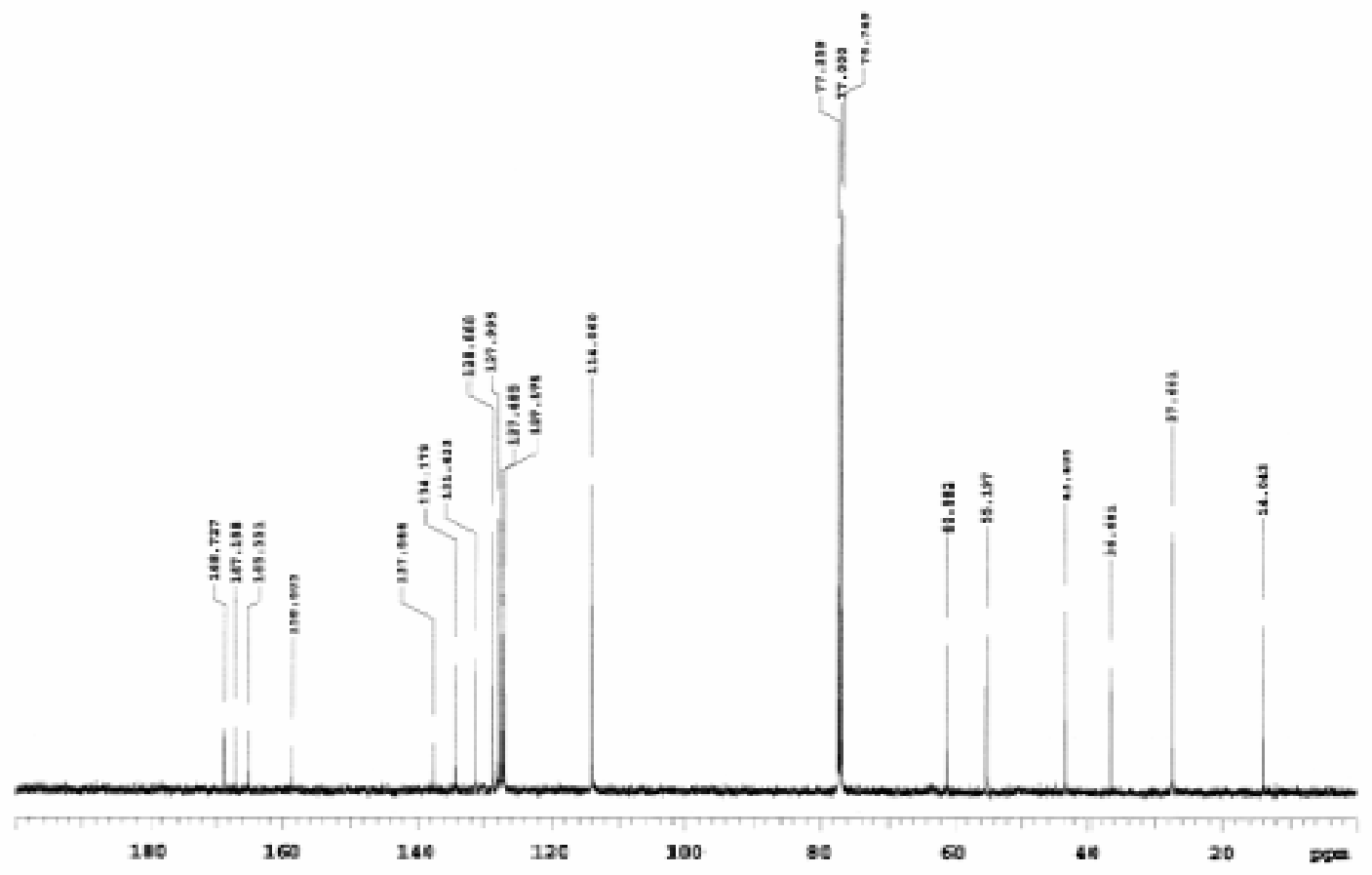

Ethyl 2-(4-(4-methoxybenzyl)-1-benzyl-5-(naphthalen-3-yl)-3,6-dioxopiperazin-2-yl)acetate (7) ${ }^{\text {b }}$<smiles>CCOC(=O)C(C)(C)C1(C)C(=O)N(Cc2ccc(OC)cc2)C(C)(c2ccc3ccccc3c2)C(=O)N1Cc1ccccc1</smiles>

7

${ }^{1} \mathrm{H}$ NMR $\left(400 \mathrm{MHz}, \mathrm{CD}_{2} \mathrm{Cl}_{2}\right): \delta$ 7.86-7.98 $(\mathrm{m}, 3 \mathrm{H}$, aryl), $7.76(\mathrm{~s}, 1 \mathrm{H}$, aryl), 7.53-7.61 (m, $2 \mathrm{H}$, aryl), 7.38-7.45 (m, 1H, aryl), 7.24-7.35 (m, 3H, aryl), 7.05-7.19 (m, 4H, aryl), 6.80$6.89(\mathrm{~m}, 2 \mathrm{H}$, aryl), 5.43 (d, 1H, J = 14.6 Hz, Bn), 5.16 (d, 1H, J = 15.4 Hz, Bn), 5.14 (s, $\left.1 \mathrm{H}, \mathrm{H}_{\mathrm{a}}\right), 4.33-4.38\left(\mathrm{~m}, 1 \mathrm{H}, \mathrm{H}_{\mathrm{b}}\right), 3.98-4.17\left(\mathrm{~m}, 3 \mathrm{H}, \mathrm{Bn}\right.$ and $-\mathrm{OC}_{2} \mathrm{CH}_{3}$ overlap), $3.8(\mathrm{~s}$, $\left.3 \mathrm{H}, \mathrm{OCH}_{3}\right), 3.42(\mathrm{~d}, 1 \mathrm{H}, J=14.6 \mathrm{~Hz}, \mathrm{Bn}), 3.28\left(\mathrm{dd}, 1 \mathrm{H}, J=3.2,17.4 \mathrm{~Hz}, \mathrm{H}_{\mathrm{c}}\right), 2.98$ (dd, $\left.1 \mathrm{H}, J=5.3,17.4 \mathrm{~Hz}, \mathrm{H}_{\mathrm{c}}\right), 1.20\left(\mathrm{t}, 3 \mathrm{H}, J=7.3 \mathrm{~Hz}, \mathrm{CH}_{3}\right)$.

(b) NOe data obtained from $\mathbf{7}$ corresponds well with published data indicating a trans relationship between napthal and methylene ethyl ester. See: Davies, S. G.; Garner, C.; Ouzman, J. V. A.; Roberts, P. M.; Smith, A. D.; Snow, E. J.; Thomson, J. E.; Tamayo, J. A.; Vickers, R. J. Org. Biomol. Chem., 2007, 5, 2138-2147. 
${ }^{13} \mathrm{C}$ NMR $\left(100 \mathrm{MHz}, \mathrm{CD}_{2} \mathrm{Cl}_{2}\right): \delta 170.1,166.1,165.7,159.7,136.1,135.1,133.7,133.6$, $130.5,129.4,129.2,128.4,128.3,128.2,128.0,127.8,127.6,126.9,125.1,63.2,61.4$, 55.6, 55.5, 47.2, 46.8, 35.3, 14.2.

HRMS: EIMS $\left(\mathrm{M}^{+}\right)$calcd for $\mathrm{C}_{38} \mathrm{H}_{32} \mathrm{~N}_{2} \mathrm{O}_{5}$ 536.2311, found 536.2313.

\section{${ }^{1}$ H-NMR (Compound 7)}
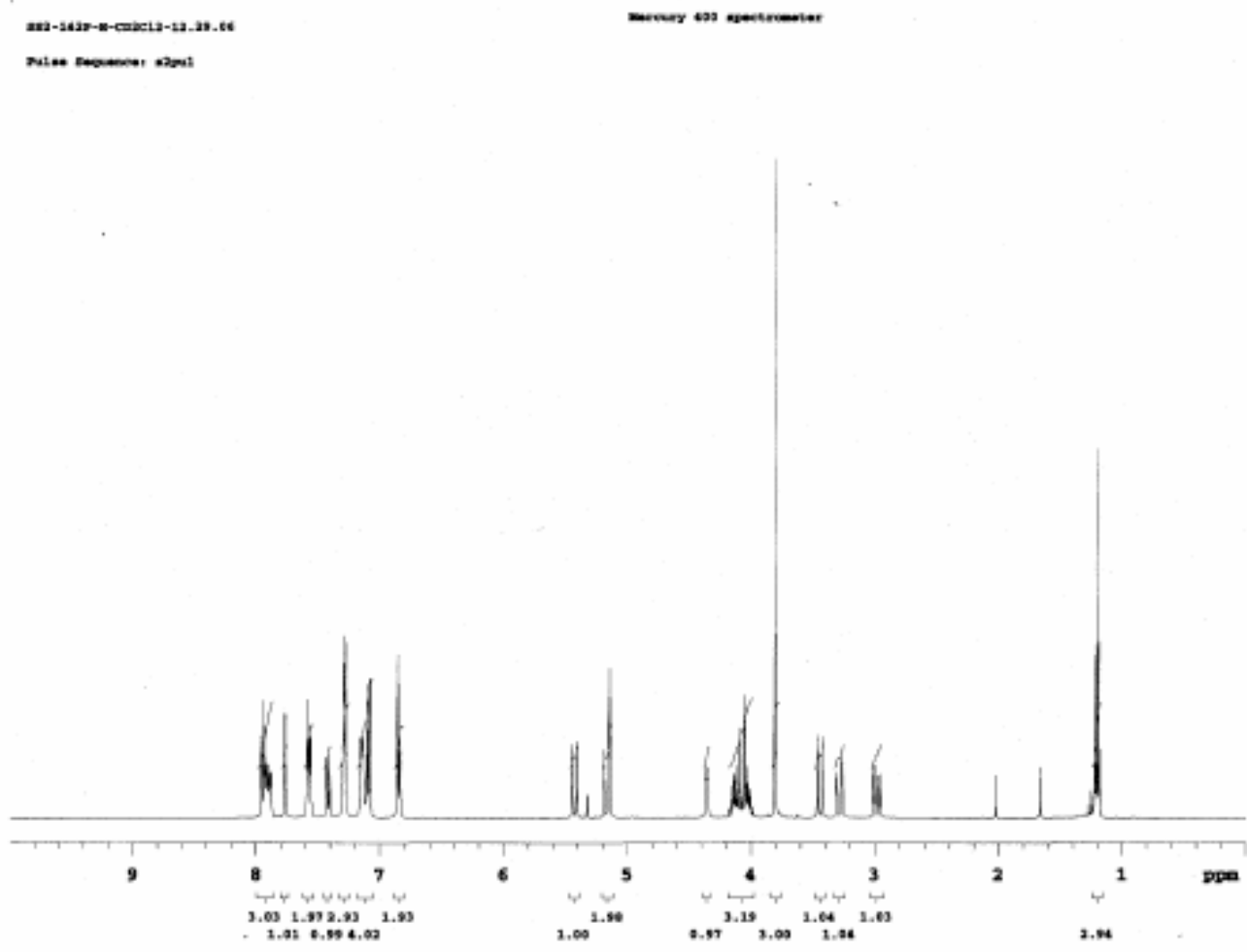


\section{${ }^{13}$ C-NMR (Compound 7)}

I

ma-16:a-c-enวe1a-13.39.06

nutas anguese: sapas

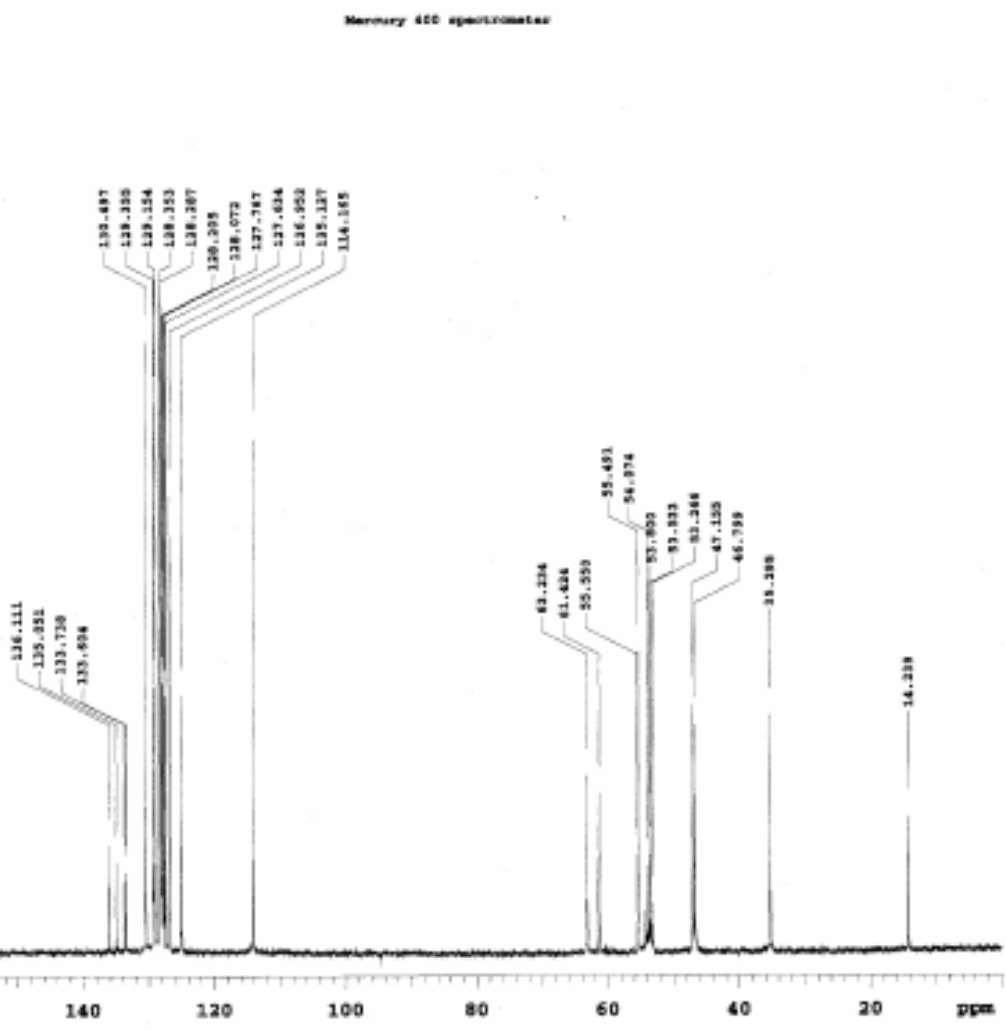

(E)-ethyl 4-((2-(benzylamino)-1-(naphthalen-2-yl)-2-oxoethyl)(4-methoxybenzyl)amino)-4-oxobut-2enoate (8)

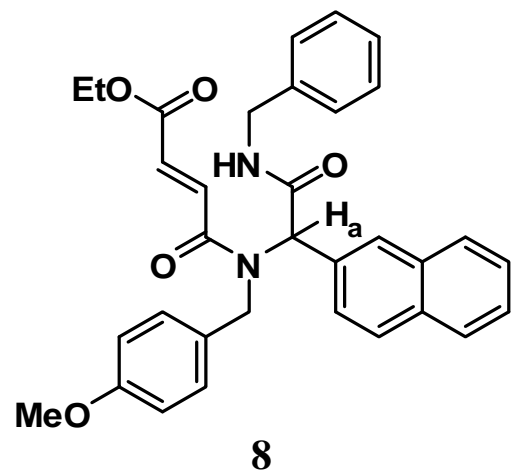

${ }^{1} \mathrm{H}$ NMR $\left(500 \mathrm{MHz}, \mathrm{CDCl}_{3}\right): \delta$ 7.68-7.83 (m, 4H), 7.45-7.53 (m, 3H), 7.36-7.43 (m, 1H),

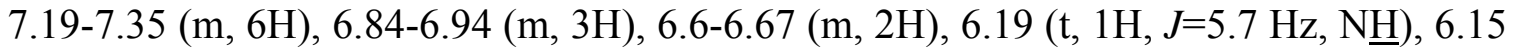
$\left(\mathrm{s}, 1 \mathrm{H}, \mathrm{H}_{\mathrm{a}}\right), 4.79(\mathrm{~d}, 1 \mathrm{H}, J=17.8 \mathrm{~Hz}), 4.58(\mathrm{~d}, 1 \mathrm{H}, J=17.8 \mathrm{~Hz}), 4.49$ (ddd, $1 \mathrm{H}, J=5.7,15.2$, $38.8 \mathrm{~Hz}$ ), 4.19 (q, $2 \mathrm{H}, J=7.3 \mathrm{~Hz},-\mathrm{OCH}_{2} \mathrm{CH}_{3}$ ), 3.68 (s, $3 \mathrm{H},-\mathrm{OCH}_{3}$ ), 1.26 (t, $3 \mathrm{H}, J=7.3 \mathrm{~Hz}$, $\left.-\mathrm{OCH}_{2} \mathrm{CH}_{3}\right)$

${ }^{13} \mathrm{C}$ NMR $\left(125 \mathrm{MHz}, \mathrm{CDCl}_{3}\right): \delta 169.1,166.3,165.2,158.5,137.8,133.8,132.9,132.8$, 132.2, 131.6, 129.2, 129.1, 128.9, 128.5, 127.9, 127.5, 127.2, 126.8, 126.6, 126.3, 113.7, $63.2,63.1,60.9,49.5,43.5,13.9$. 
HRMS: EIMS $\left(\mathrm{M}^{+}\right)$calcd for $\mathrm{C}_{33} \mathrm{H}_{32} \mathrm{~N}_{2} \mathrm{O}_{5}$ 536.2311, found 536.2318.

\section{$\underline{{ }^{1} \text { H-NMR (Compound 8) }}$}
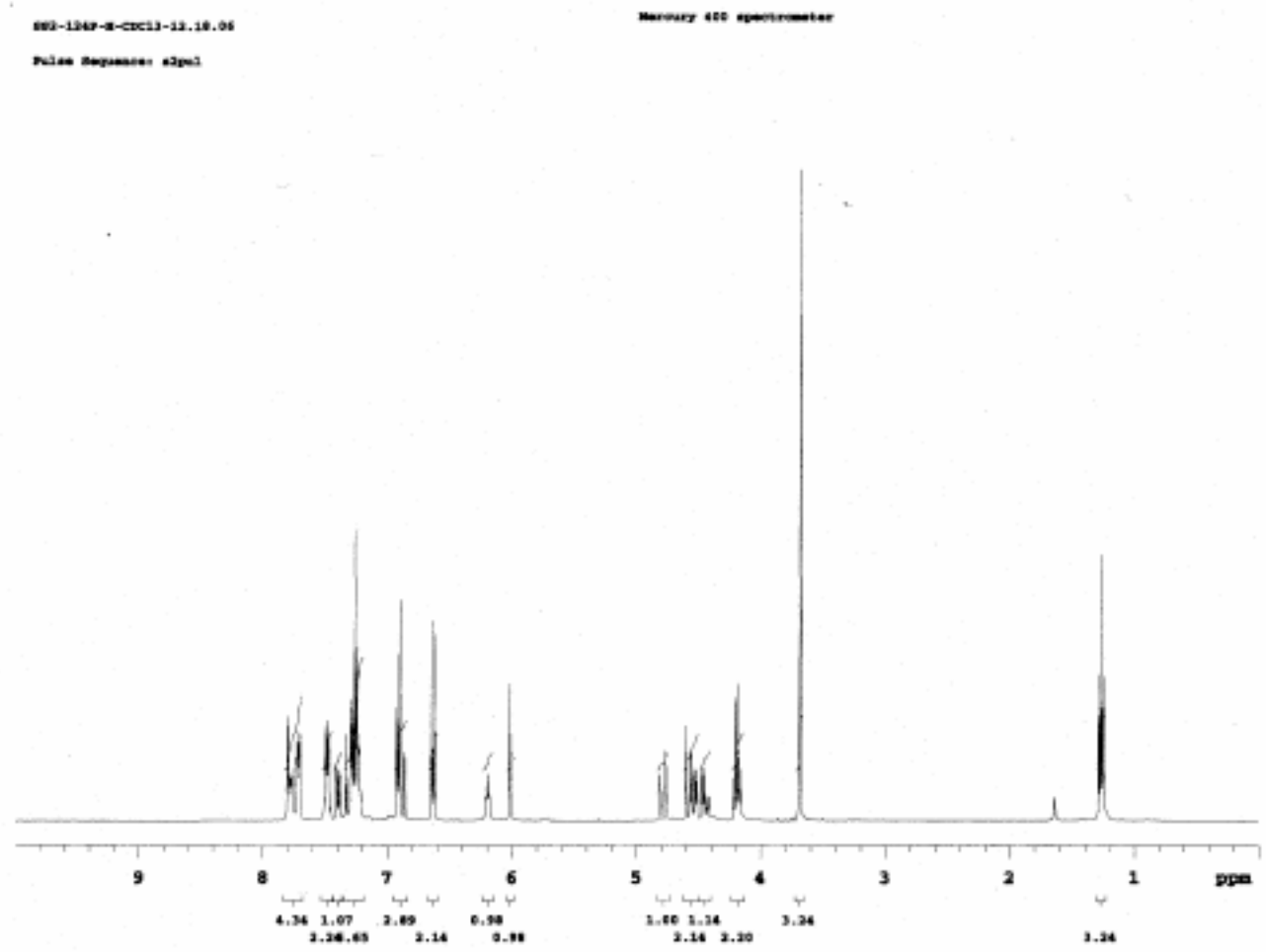

\section{${ }^{13}$ C-NMR (Compound 8)}

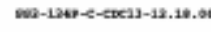

meroury 460 movotroneter

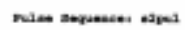

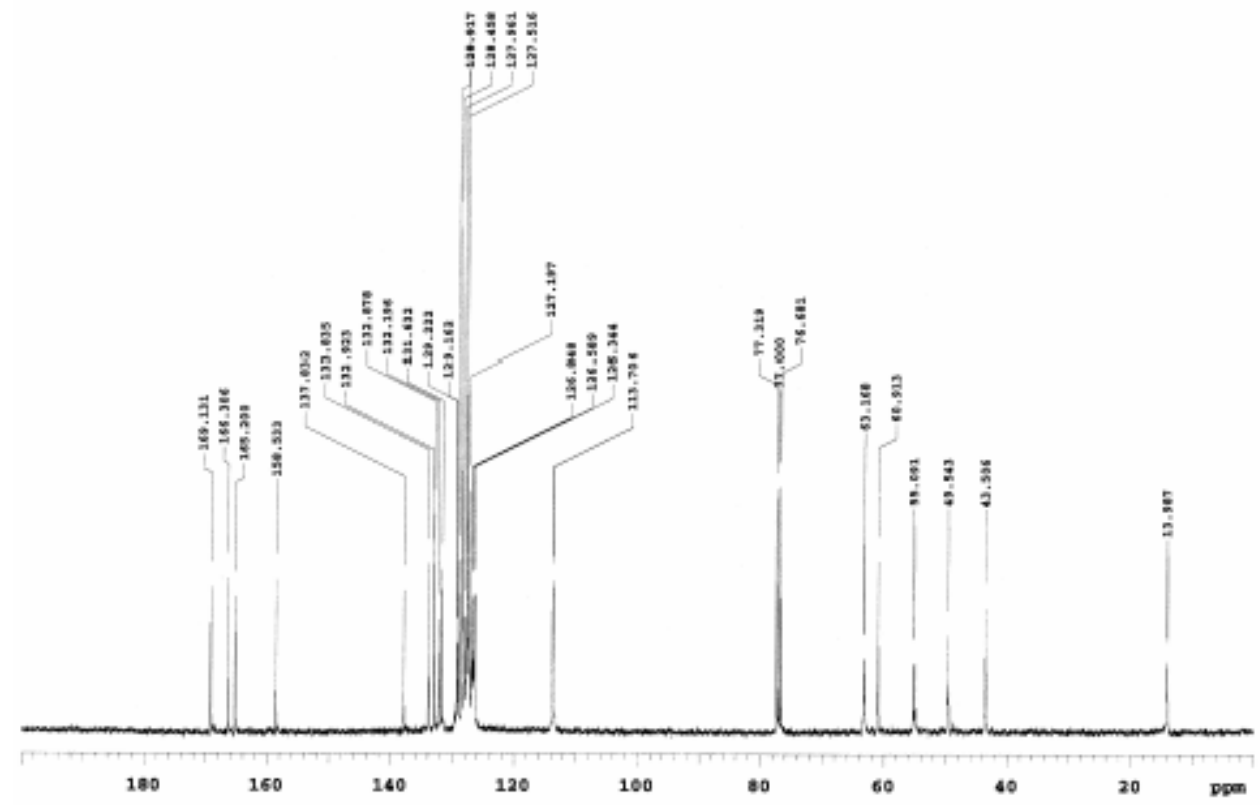


Ethyl 2-(5-tert-butyl-1,4-dibenzyl-3,6-dioxopiperazin-2-yl)acetate (9)

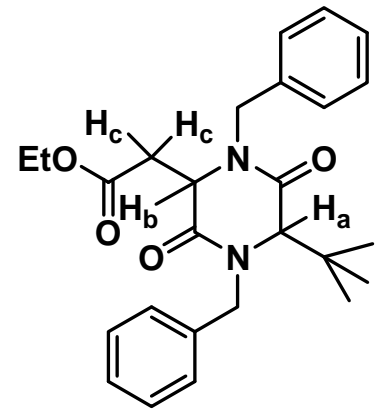

9

${ }^{1} \mathrm{H}$ NMR (400 MHz, $\mathrm{CDCl}_{3}$ ): $\delta$ 7.20-7.37 (m, 10H, aryl), $5.64(\mathrm{~d}, 1 \mathrm{H}, J=15.4 \mathrm{~Hz}, \mathrm{Bn})$, $5.11(\mathrm{~d}, 1 \mathrm{H}, J=15.4 \mathrm{~Hz}, \mathrm{Bn}), 4.49\left(\mathrm{dd}, 1 \mathrm{H}, J=4.1,5.7 \mathrm{~Hz}, \mathrm{H}_{\mathrm{b}}\right), 4.29(\mathrm{~d}, 1 \mathrm{H}, J=15.4 \mathrm{~Hz}$, $\mathrm{Bn}), 4.0-4.15\left(\mathrm{~m}, 2 \mathrm{H},-\mathrm{OCH}_{2} \mathrm{CH}_{3}\right), 3.29(\mathrm{~d}, 1 \mathrm{H}, J=15.4 \mathrm{~Hz}, \mathrm{Bn}), 3.78\left(\mathrm{~s}, 1 \mathrm{H}, \mathrm{H}_{\mathrm{a}}\right), 3.16$ $\left(\mathrm{dd}, 1 \mathrm{H}, J=4.1,17 \mathrm{~Hz}, \mathrm{H}_{\mathrm{c}}\right), 2.96\left(\mathrm{dd}, 1 \mathrm{H}, J=5.7,17.4 \mathrm{~Hz}, \mathrm{H}_{\mathrm{c}}\right), 1.20(\mathrm{t}, 3 \mathrm{H}, J=7.3 \mathrm{~Hz}$, $\left.\mathrm{CH}_{3}\right), 1.11$ (s, 9H, t-butyl).

${ }^{13} \mathrm{C}$ NMR $\left(100 \mathrm{MHz} \mathrm{CDCl}_{3}\right): \delta 169.9,167.7,166.6,136.5,135.6,128.8,128.7,127.9$, $127.8,127.7,127.6,68.1,60.8,55.6,51.4,46.9,40.0,34.8,28.3,14$.

HRMS: EIMS $\left(\mathrm{M}^{+}\right)$calcd for $\mathrm{C}_{26} \mathrm{H}_{32} \mathrm{~N}_{2} \mathrm{O}_{4} 436.2362$, found 436.2372.

\section{${ }^{1}$ H-NMR (Compound 9)}
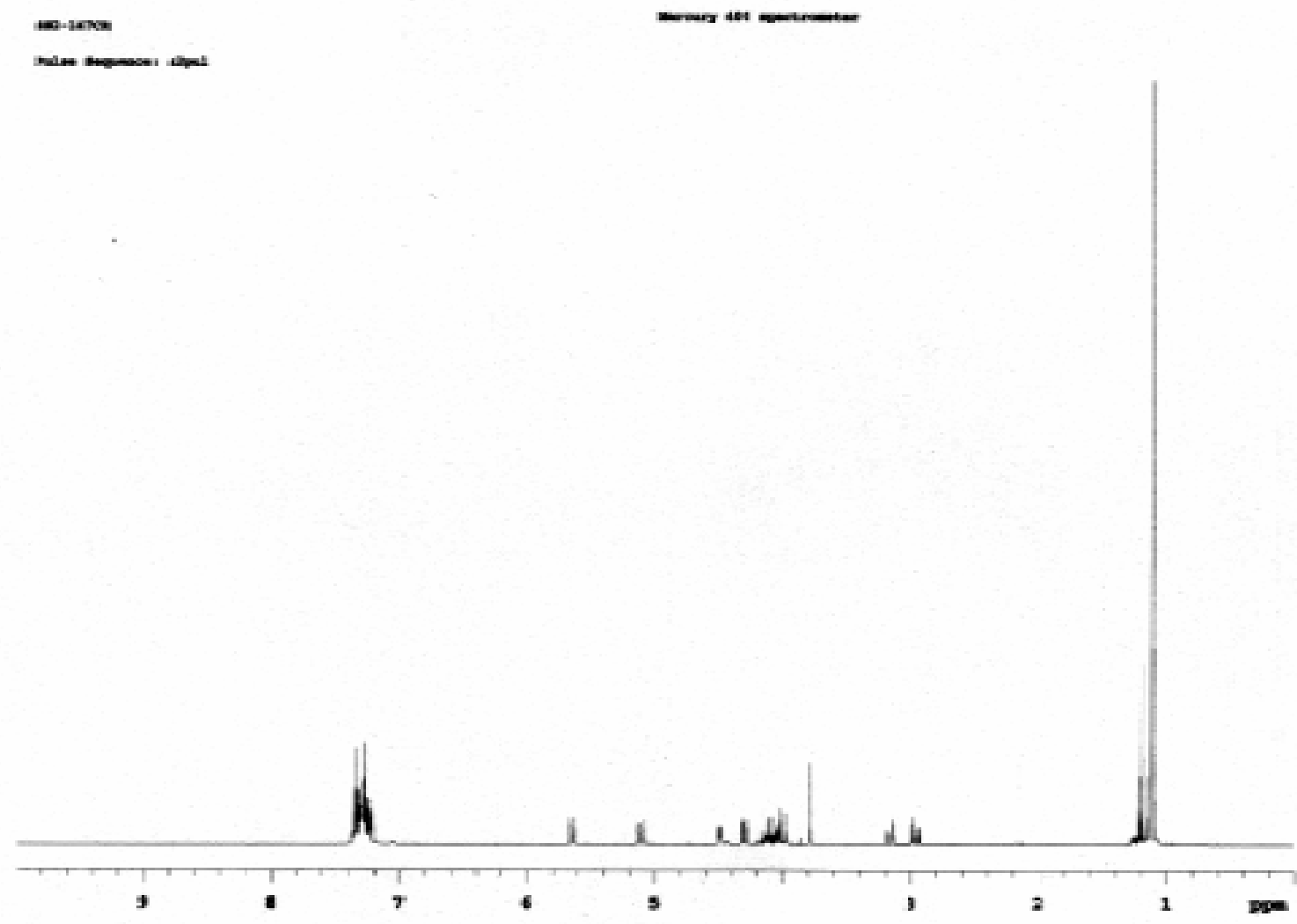


\section{${ }^{13}$ C-NMR (Compound 9)}
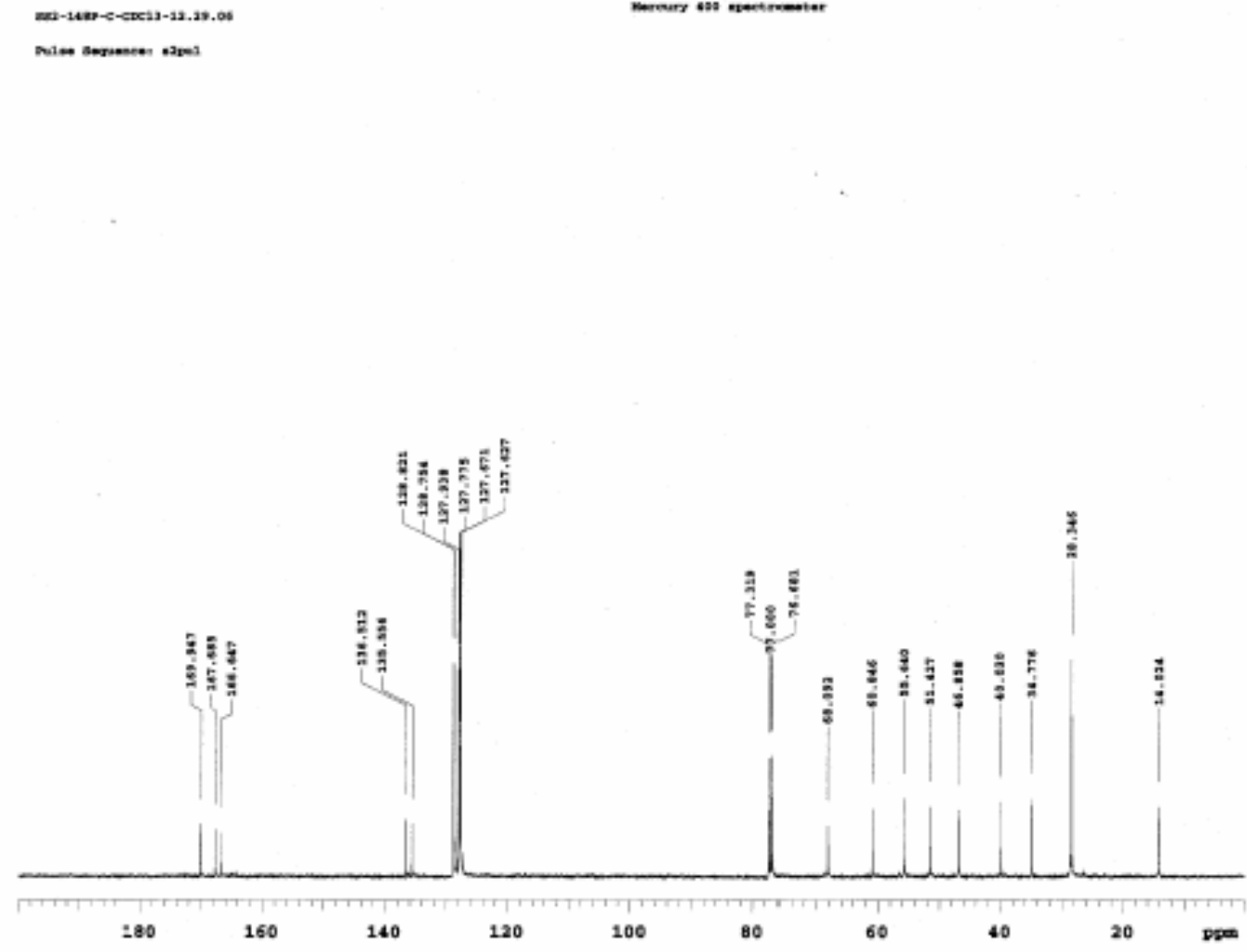

(E)-ethyl 4-(benzyl(1-(benzylamino)-3,3-dimethyl-1-oxobutan-2-yl)amino)-4-oxobut-2-enoate (10)<smiles>CCOC(=O)/C=C/C(=O)N(Cc1ccccc1)C(C)(C(=O)NCc1ccccc1)C(C)(C)C</smiles>

10

${ }^{1} \mathrm{H}$ NMR (400 MHz, $\left.\mathrm{CDCl}_{3}\right): \delta 7.74(\mathrm{t}, 1 \mathrm{H}, J=5.7 \mathrm{~Hz}, \mathrm{NH}), 7.02-7.28(\mathrm{~m}, 11 \mathrm{H}$, aryl), $6.47(\mathrm{~d}, 1 \mathrm{H}, J=14.6 \mathrm{~Hz}), 5.47\left(\mathrm{~d}, 1 \mathrm{H}, J=17.8 \mathrm{~Hz}\right.$, benzyl), $5.31\left(\mathrm{~s}, 1 \mathrm{H}, \mathrm{H}_{\mathrm{a}}\right), 4.78(\mathrm{~d}, 1 \mathrm{H}$, $J=17.8 \mathrm{~Hz}$, benzyl), $4.31(\mathrm{dd}, 1 \mathrm{H}, J=5.6,14.6 \mathrm{~Hz}$, benzyl), 4.09-4.16 (m, 3H, $\mathrm{OC}_{2} \mathrm{CH}_{3}$ and benzyl overlap), 1.22 (t, 3H, $\left.J=7.3 \mathrm{~Hz},-\mathrm{OCH}_{2} \underline{\mathrm{C}}_{3}\right), 1.10$ (s, 9H, t-butyl).

${ }^{13} \mathrm{C}$ NMR $\left(100 \mathrm{MHz} \mathrm{CDCl}_{3}\right): \delta 168.6,166.9,165.0,138.4,137.7,134.1,131.2,131.1$, $128.5,128.4,127.8,127.1,126.9,125.7,62.9,60.8,49.6,43.2,36.8,27.5,13.9$

MS: EIMS $(\mathrm{M}+\mathrm{H})$ calcd for $\mathrm{C}_{26} \mathrm{H}_{32} \mathrm{~N}_{2} \mathrm{O}_{4} 437.2396$, found 437.2. 
$\underline{{ }^{1} \text { H-NMR (Compound 10) }}$
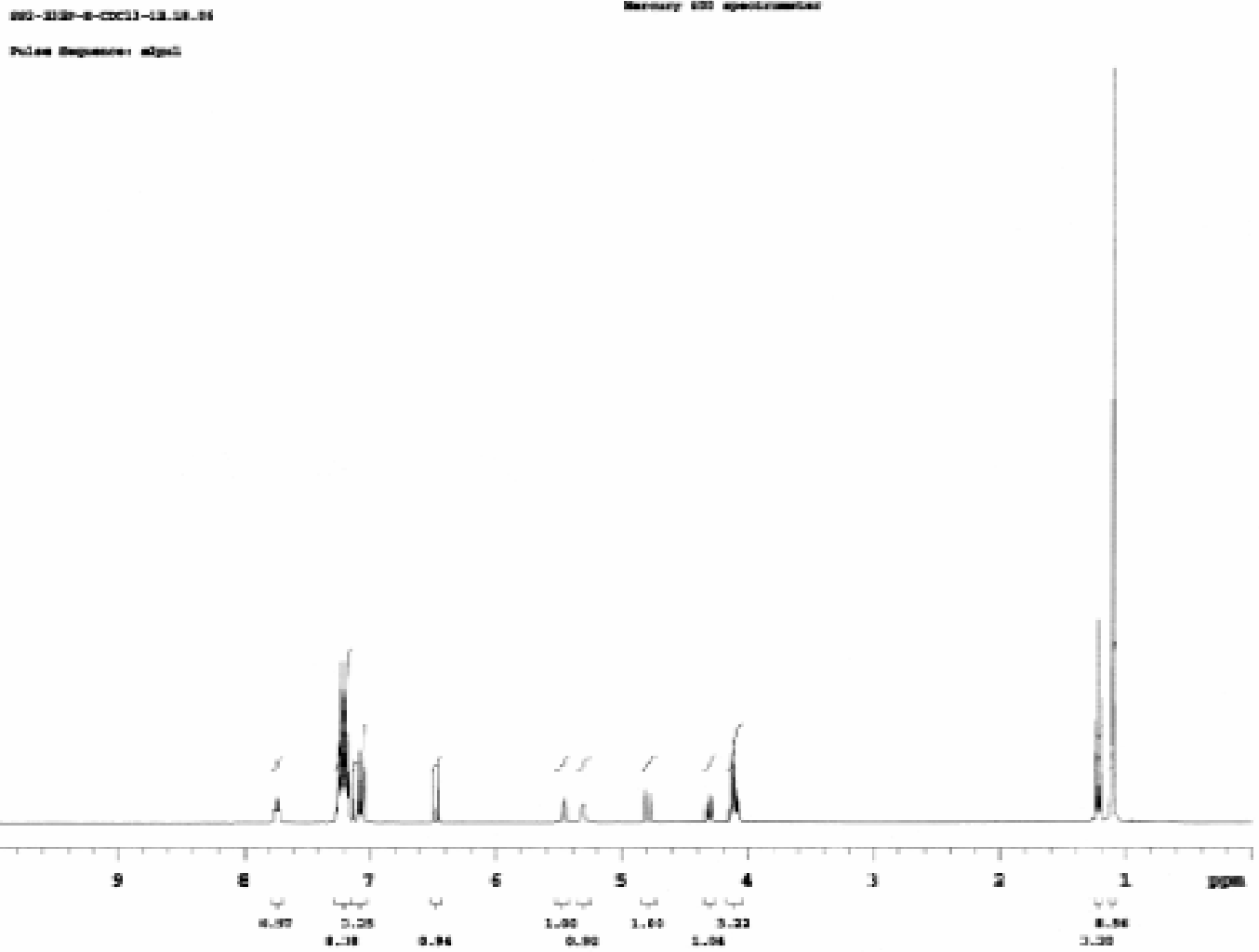

${ }^{13}$ C-NMR (Compound 10)

asz-13ap-e-crecis-12.16.46

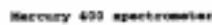

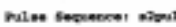

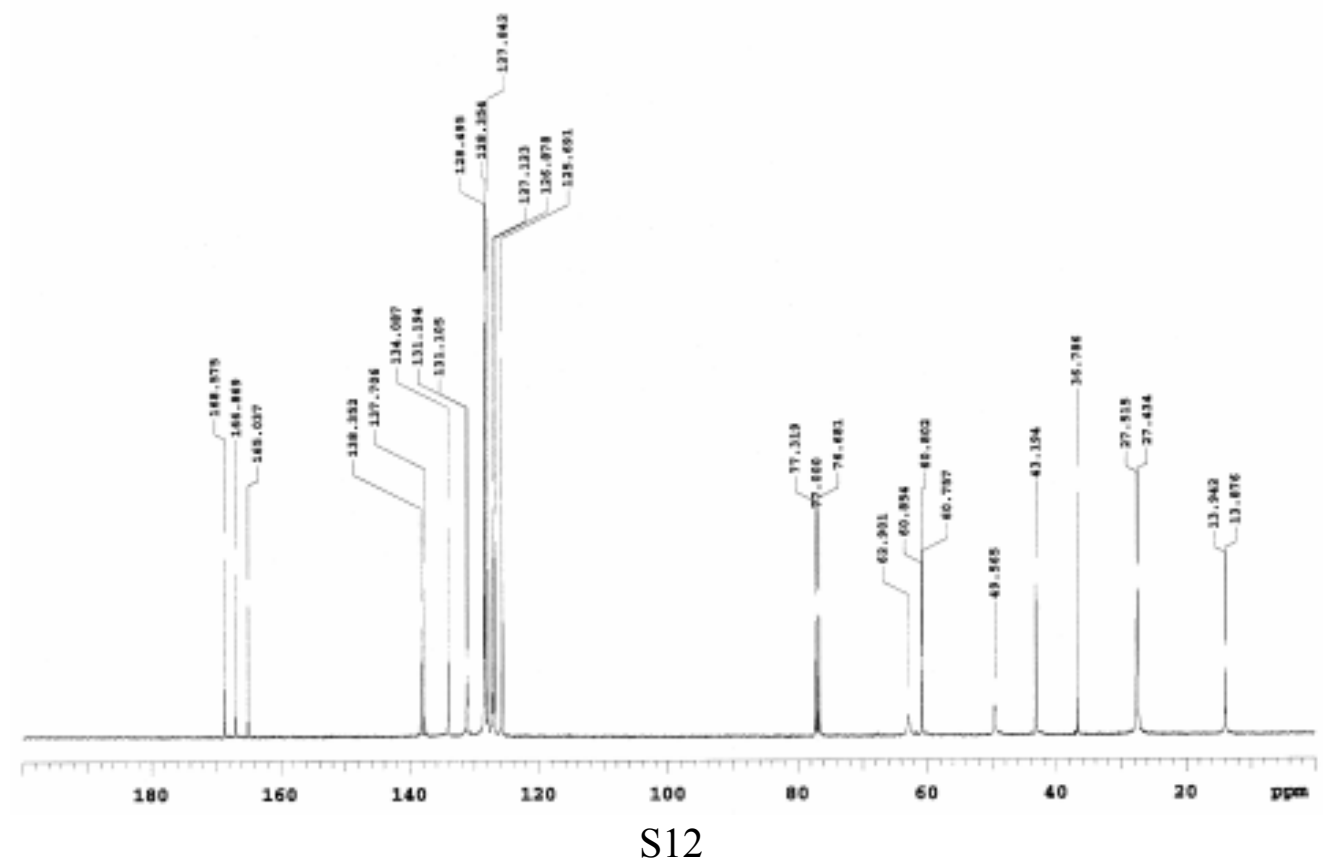


Ethyl 2-(5-tert-butyl-1-benzyl-4-(cyclohexylmethyl)-3,6-dioxopiperazin-2-yl)acetate (11)

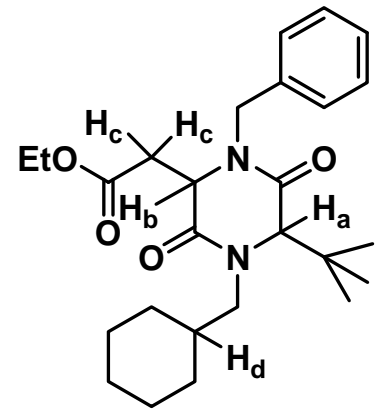

11

${ }^{1} \mathrm{H}$ NMR (400 MHz, $\left.\mathrm{CDCl}_{3}\right): \delta$ 7.21-7.35 (m, 5H, aryl), $5.14(\mathrm{~d}, 1 \mathrm{H}, J=15.4 \mathrm{~Hz}, \mathrm{Bn})$, $4.32\left(\mathrm{dd}, 1 \mathrm{H}, J=3.3,5.7 \mathrm{~Hz}, \mathrm{H}_{\mathrm{b}}\right), 4.23-4.30(\mathrm{~m}, 2 \mathrm{H}), 3.99-4.17(\mathrm{~m}, 3 \mathrm{H}), 3.77\left(\mathrm{~s}, 1 \mathrm{H}, \mathrm{H}_{\mathrm{a}}\right)$, $3.15\left(\mathrm{dd}, 1 \mathrm{H}, J=4.1,17.4 \mathrm{~Hz}, \mathrm{H}_{\mathrm{c}}\right), 2.9\left(\mathrm{dd}, 1 \mathrm{H}, J=6.5,17.0 \mathrm{~Hz}, \mathrm{H}_{\mathrm{c}}\right), 2.50(\mathrm{dd}, 1 \mathrm{H}, J=$ 9.7, $13.8 \mathrm{~Hz}), 1.45-1.85$ (m, 8H, cyclohexyl), 1.21 (t, 3H, J=7.3 Hz, $\left.\mathrm{CH}_{3}\right), 1.05$ (s, 9H, t-butyl), 0.75-0.93 (m, 2H, cyclohexyl).

${ }^{13} \mathrm{C}$ NMR $\left(100 \mathrm{MHz}, \mathrm{CDCl}_{3}\right): \delta 169.8,167.1,166.8,136.5,128.8,127.8,127.7,71.5$, $60.8,56.9,55.9,46.9,40.2,35.6,35.1,31.2,30.6,28.0,26.4,25.7,14.1$.

HRMS: EIMS $\left(\mathrm{M}^{+}\right)$calcd for $\mathrm{C}_{26} \mathrm{H}_{38} \mathrm{~N}_{2} \mathrm{O}_{4} 442.2832$, found 442.2836.

\section{$\underline{{ }^{1} \text { H-NMR (Compound 11) }}$}
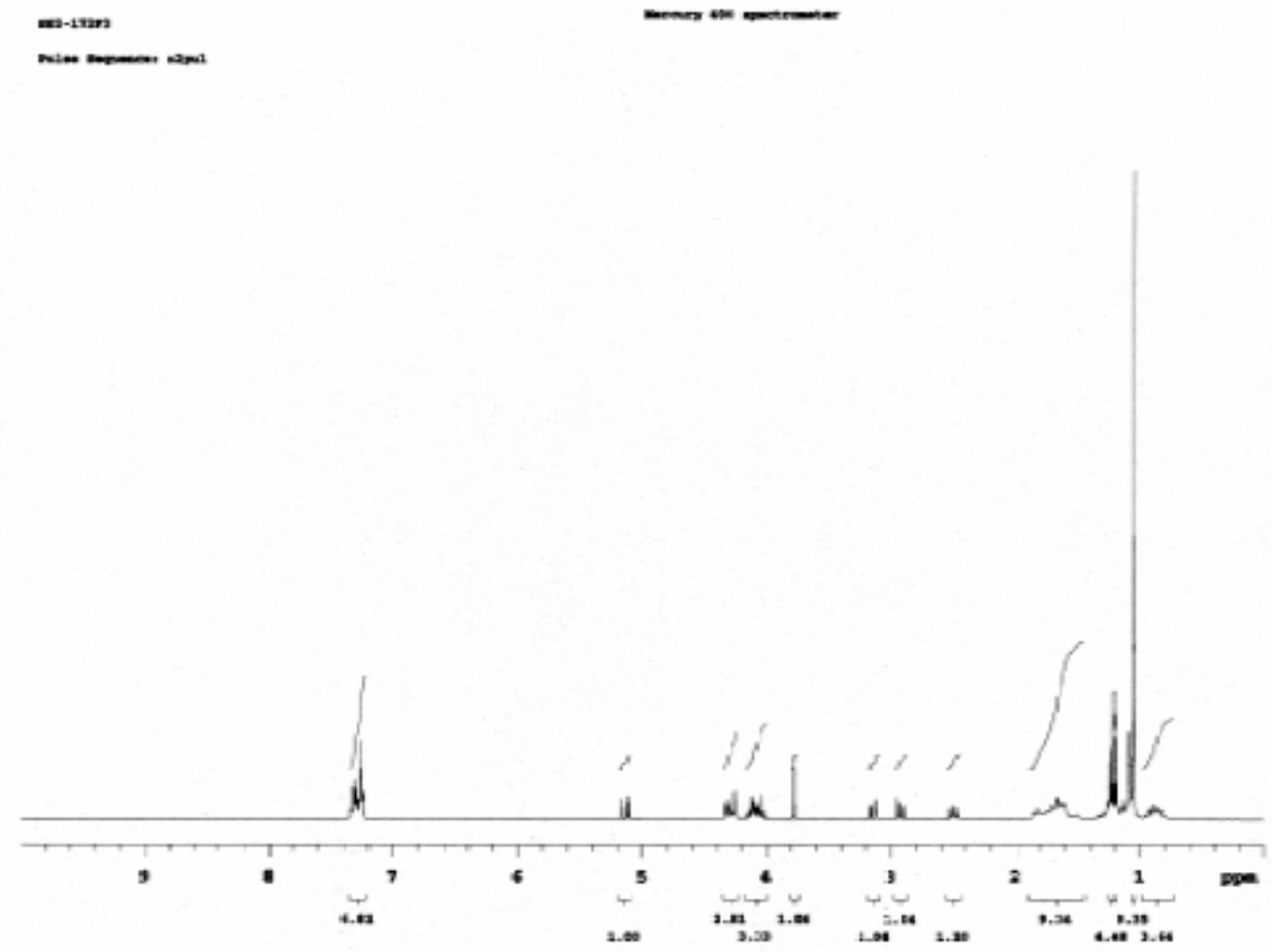


\section{${ }^{13}$ C-NMR (Compound 11)}

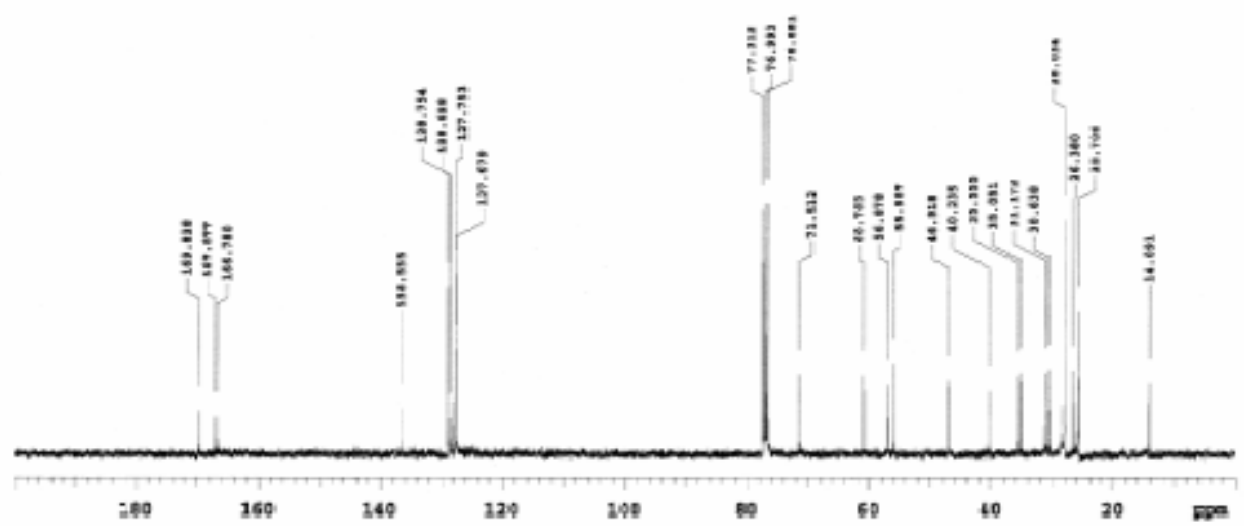

(E)-ethyl 4-((1-(benzylamino)-3,3-dimethyl-1-oxobutan-2-yl)(cyclohexylmethyl)amino)-4-oxobut-2enoate (12)<smiles>CCOC(=O)/C=C/C(=O)N(CC1CCCCC1)C(C)(C(=O)NCc1ccccc1)C(C)(C)C</smiles>

12

${ }^{1} \mathrm{H}$ NMR (400 MHz, $\left.\mathrm{CDCl}_{3}\right): \delta 7.35(\mathrm{~d}, 1 \mathrm{H}, J=15.4 \mathrm{~Hz},-\mathrm{CH}=\mathrm{CH}-), 7.19-7.31(\mathrm{~m}, 5 \mathrm{H}$, aryl), $6.69(\mathrm{~d}, 1 \mathrm{H}, J=15.4 \mathrm{~Hz},-\mathrm{CH}=\mathrm{CH}-), 4.47$ (dd, $1 \mathrm{H}, J=6.5,14.6 \mathrm{~Hz}$, benzyl), 4.17$4.32\left(\mathrm{~m}, 4 \mathrm{H},-\mathrm{OCH}_{2} \mathrm{CH}_{3}\right.$, benzyl and $\mathrm{H}_{\mathrm{a}}$ overlap), 3.72 (bs, $1 \mathrm{H}$, cyclohexylmethyl), 3.25 (bs, $1 \mathrm{H}$, cyclohexylmethyl), 1.40-1.72 (m, 8H, cyclohexyl), 1.29 (t, 3H, J = 7.3 Hz, $\mathrm{OCH}_{2} \underline{\mathrm{C}}_{3}$ ), 1.06 (s, 9H, t-butyl), 0.70-0.94 (m, 3H, cyclohexyl).

${ }^{13} \mathrm{C}$ NMR $\left(100 \mathrm{MHz}, \mathrm{CDCl}_{3}\right): \delta 169.5,166.7,165.5,137.9,134.6,131.1,128.5,127.8$, $127.2,61.1,43.4,36.8,30.8,29.6,28.1,26.1,25.8,25.7,14.1$.

MS: EIMS $(\mathrm{M}+\mathrm{H})$ calcd for $\mathrm{C}_{26} \mathrm{H}_{38} \mathrm{~N}_{2} \mathrm{O}_{4} 443.2832$, found 443.3 
$\underline{{ }^{1} \text { H-NMR (Compound 12) }}$

m-รtaca

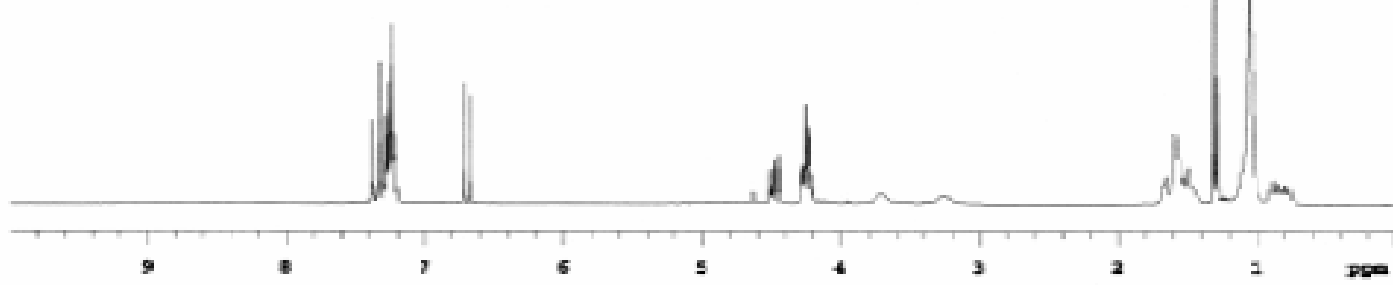

${ }^{13}$ C-NMR (Compound 12)

centions specters

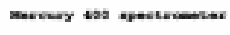

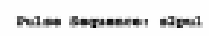

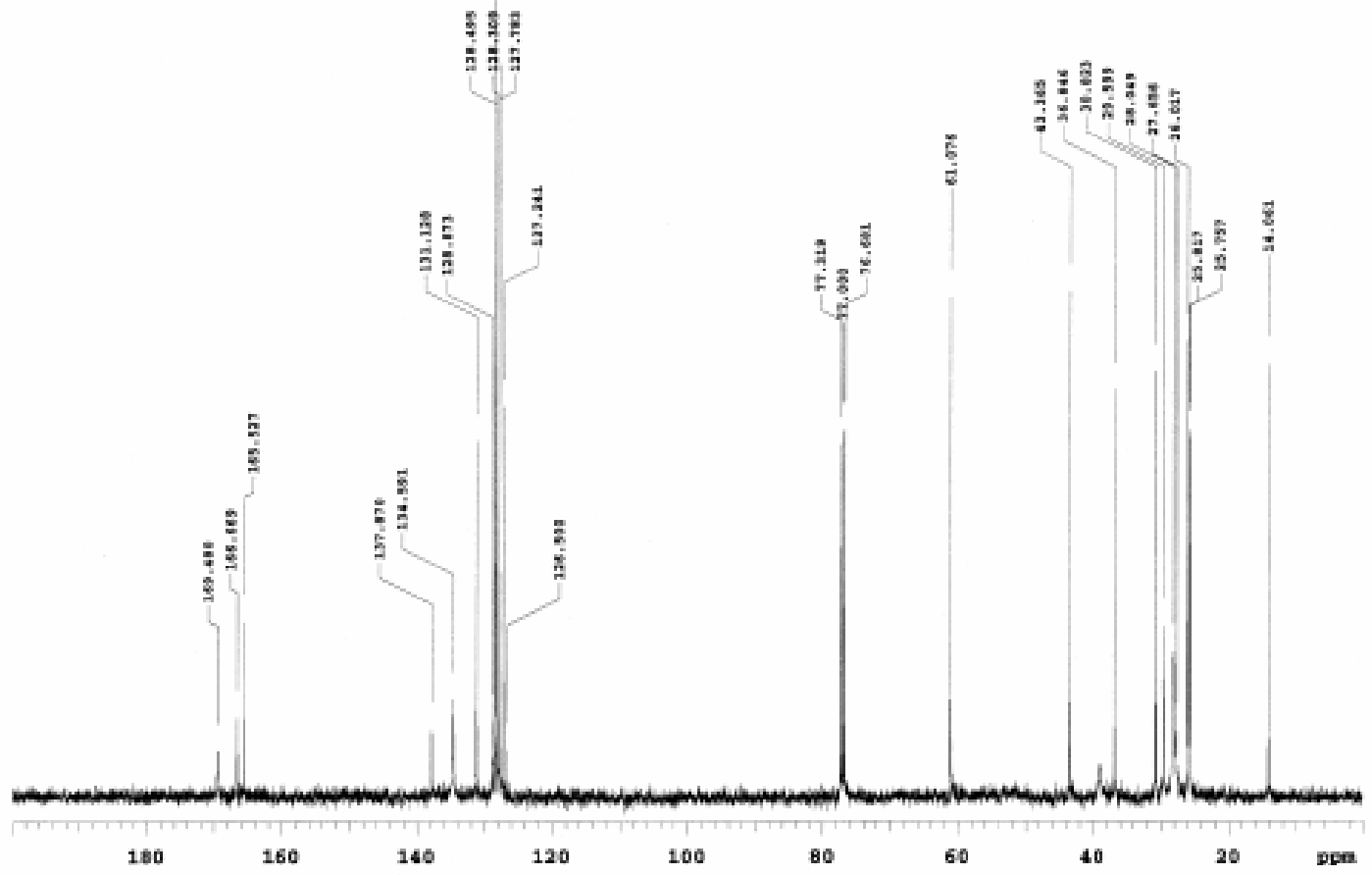


Ethyl 2-(4-benzyl-5-isopropyl-1-(4-methoxyphenyl)-3,6-dioxopiperazin-2-yl)acetate (13)

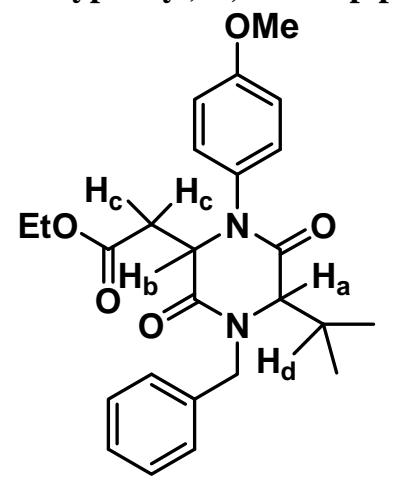

13

${ }^{1} \mathrm{H}$ NMR (400 MHz, $\mathrm{CD}_{2} \mathrm{Cl}_{2}$ ): $\delta$ 7.30-7.45 (m, 5H, aryl), 7.03-7.10 (m, 2H, aryl), 6.92$6.98\left(\mathrm{~m}, 2 \mathrm{H}\right.$, aryl), $5.44(\mathrm{~d}, 1 \mathrm{H}, J=15.4 \mathrm{~Hz}, \mathrm{Bn}), 4.81\left(\mathrm{t}, 1 \mathrm{H}, J=4.1 \mathrm{~Hz}, \mathrm{H}_{\mathrm{b}}\right), 4.09$ (d, $1 \mathrm{H}, J=15.4 \mathrm{~Hz}, \mathrm{Bn}), 4.03$ (q, $\left.2 \mathrm{H}, J=7.3 \mathrm{~Hz},-\mathrm{OC}_{2} \mathrm{CH}_{3}\right), 3.84\left(\mathrm{~d}, 1 \mathrm{H}, J=4.9 \mathrm{~Hz}, \mathrm{H}_{\mathrm{a}}\right.$ ), $3.82\left(\mathrm{~s}, 3 \mathrm{H}, \mathrm{OCH}_{3}\right), 2.98\left(\mathrm{dd}, 1 \mathrm{H}, J=4.1,17 \mathrm{~Hz}, \mathrm{H}_{\mathrm{c}}\right), 2.64\left(\mathrm{dd}, 1 \mathrm{H}, J=5.3,17.4 \mathrm{~Hz}, \mathrm{H}_{\mathrm{c}}\right)$, 2.32-2.46 (m, 1H, H $\left.\mathrm{H}_{\mathrm{d}}\right), 1.16\left(\mathrm{t}, 3 \mathrm{H}, J=7.3 \mathrm{~Hz}, \mathrm{CH}_{3}\right), 1.12\left(\mathrm{~d}, 3 \mathrm{H}, J=6.5 \mathrm{~Hz}, \mathrm{CHC}_{3}\right)$, $1.1\left(\mathrm{~d}, 3 \mathrm{H}, J=6.5 \mathrm{~Hz}, \mathrm{CHC}_{3}\right)$.

${ }^{13} \mathrm{C}$ NMR $\left(100 \mathrm{MHz}, \mathrm{CD}_{2} \mathrm{Cl}_{2}\right): \delta 170.3,166.1,165.9,136.4,131.1,129.5,129.1,128.4$, $128.0,114.8,65.1,60.9,57.7,55.8,48.5,35.9,32.5,20.2,17.7,14.2$.

HRMS: EIMS ( $\mathrm{M}^{+}$) calcd for $\mathrm{C}_{25} \mathrm{H}_{30} \mathrm{~N}_{2} \mathrm{O}_{5} 438.2155$, found 438.2162.

${ }^{1}$ H-NMR (Compound 13)
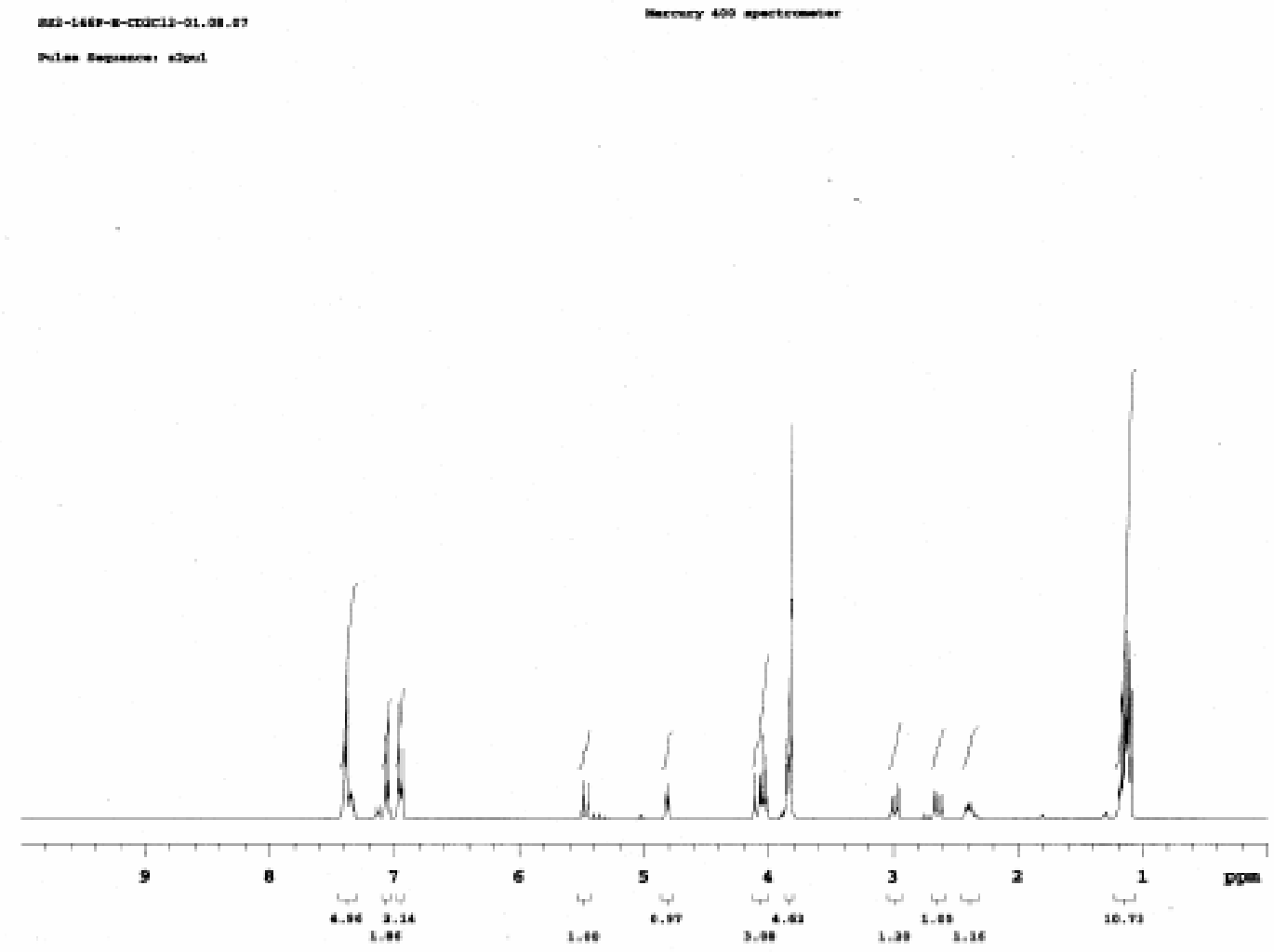


\section{${ }^{13}$ C-NMR (Compound 13)}
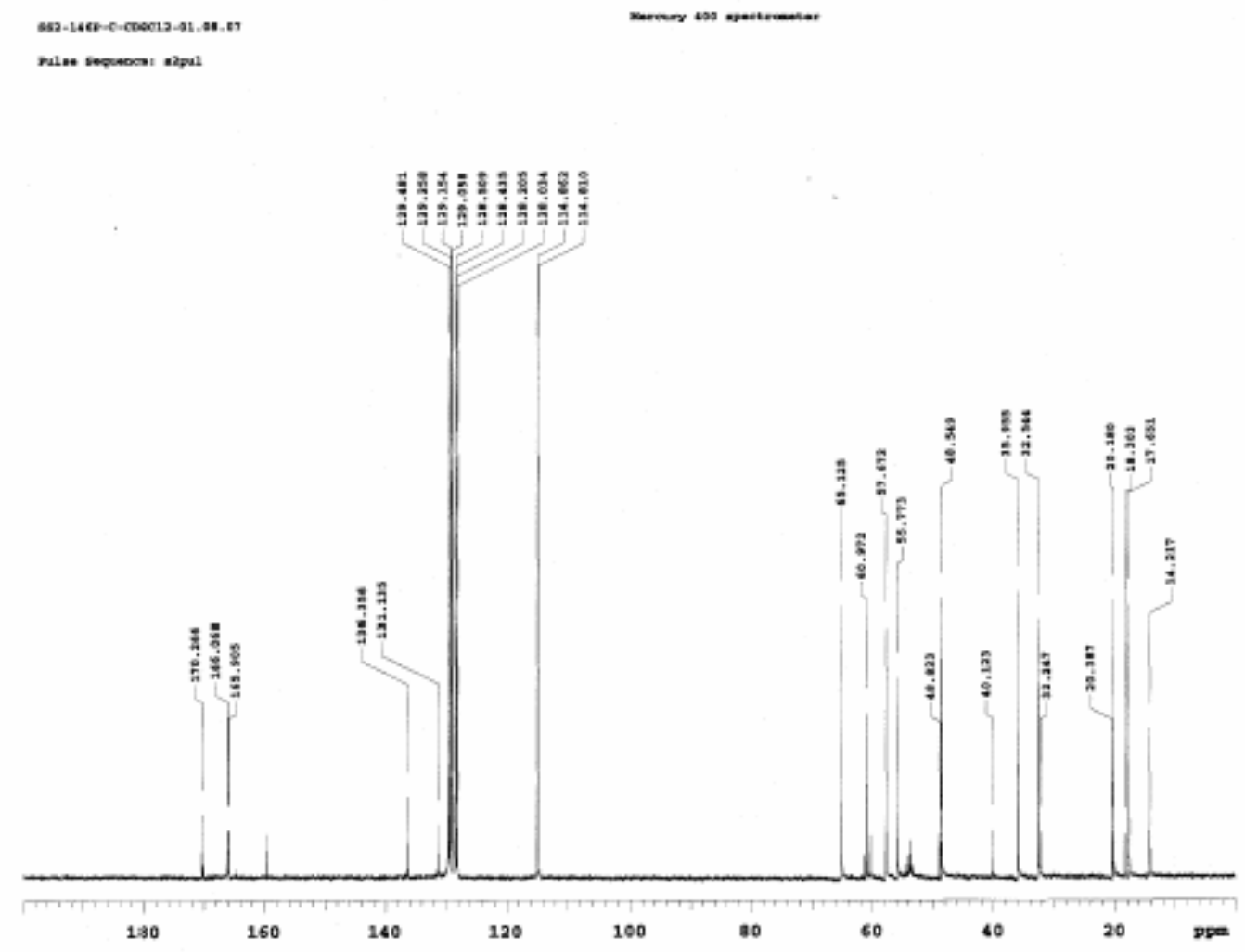

(E)-ethyl 4-(benzyl(1-(4-methoxyphenylamino)-3-methyl-1-oxobutan-2-yl)amino)-4-oxobut-2-enoate (14)<smiles>CCOC(=O)/C=C/C(=O)N(Cc1ccccc1)C(C)(C(=O)Nc1ccc(OC)cc1)C(C)C</smiles>

14

${ }^{1} \mathrm{H}$ NMR $\left(400 \mathrm{MHz}, \mathrm{CDCl}_{3}\right): \delta 8.7$ (bs, $\left.1 \mathrm{H}, \mathrm{N} \underline{\mathrm{H}}\right), 7.09-7.32(\mathrm{~m}, 8 \mathrm{H}$, aryl and $-\mathrm{C} \underline{\mathrm{H}}=\mathrm{CH}-$ overlap), 6.74-6.85 (m, 3H, aryl and $-\mathrm{CH}=\mathrm{C} \underline{\mathrm{H}}-$ overlap), $4.76(\mathrm{dd}, 2 \mathrm{H}, J=17.8 \mathrm{~Hz}), 4.55$ $\left(\mathrm{bs}, 1 \mathrm{H}, \mathrm{H}_{\mathrm{a}}\right), 4.16\left(\mathrm{q}, 2 \mathrm{H}, J=7.3 \mathrm{~Hz},-\mathrm{OC}_{2} \mathrm{CH}_{3}\right), 3.76\left(\mathrm{~s}, 3 \mathrm{H},-\mathrm{OC}_{3}\right), 2.59(\mathrm{~m}, 1 \mathrm{H}, i-$ $\operatorname{Pr}), 1.24\left(\mathrm{t}, 3 \mathrm{H}, J=7.3 \mathrm{~Hz},-\mathrm{OCH}_{2} \mathrm{CH}_{3}\right), 1.01$ (d, $\left.3 \mathrm{H}, J=6.5 \mathrm{~Hz}, i-\operatorname{Pr}\right), 0.85$ (d, $3 \mathrm{H}, J=$ $7.3 \mathrm{~Hz}, i-\operatorname{Pr})$

${ }^{13} \mathrm{C}$ NMR $\left(100 \mathrm{MHz}, \mathrm{CDCl}_{3}\right): \delta 167.4,166.9,164.9,156.2,136.6,133.7,132.3,130.6$, $128.6,127.4,126.4,121.8,113.7,66.3,60.9,49.1,26.9,19.6,18.8,13.8$.

MS: EIMS $(\mathrm{M}+\mathrm{H})$ calcd for $\mathrm{C}_{25} \mathrm{H}_{30} \mathrm{~N}_{2} \mathrm{O}_{5} 437.2155$, found 437.2 


\section{${ }^{1}$ H-NMR (Compound 14)}

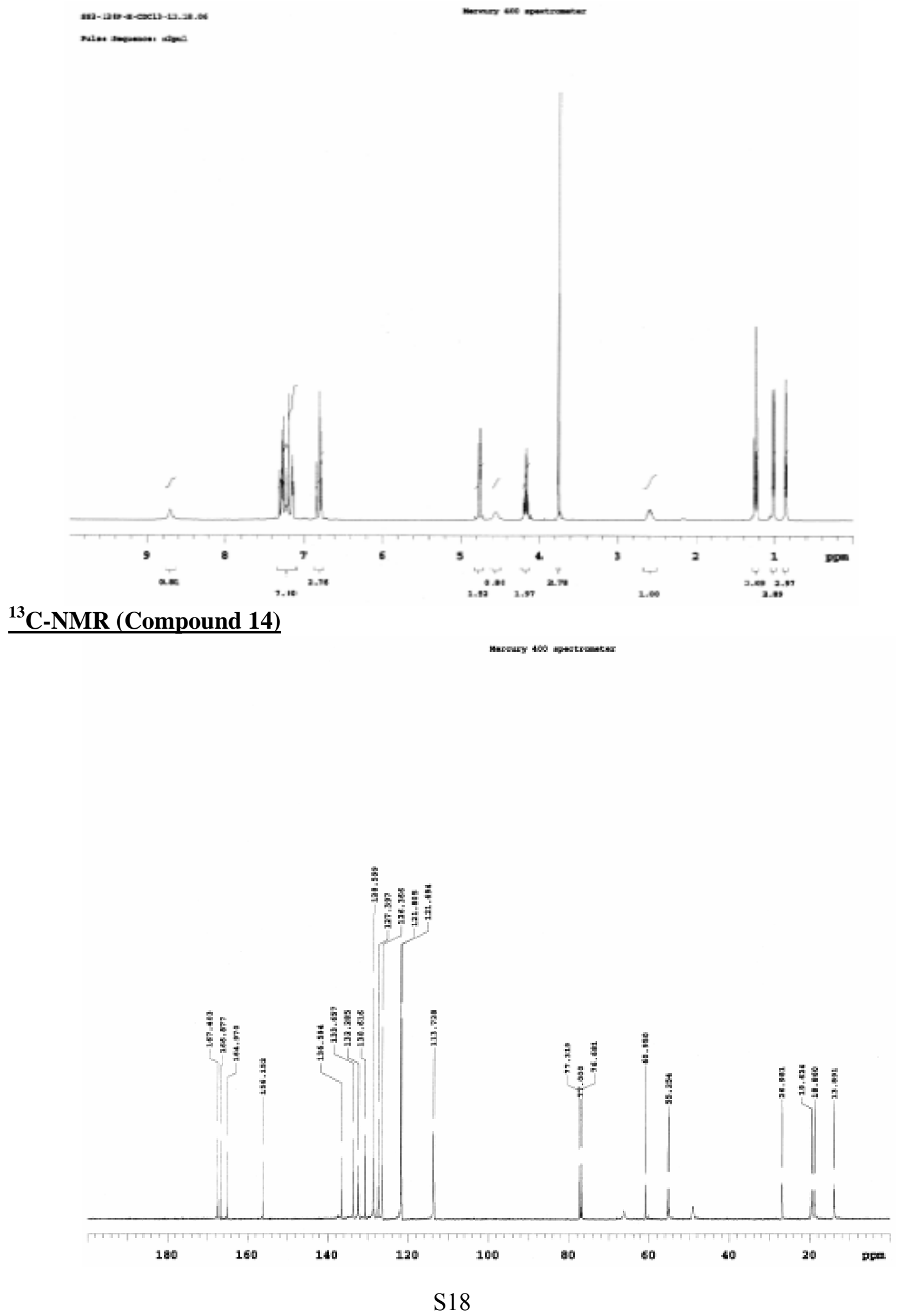


Ethyl 2-(4-(4-methoxybenzyl)-5-isopropyl-1-(4-methoxyphenyl)-3,6-dioxopiperazin-2-yl)acetate (15)

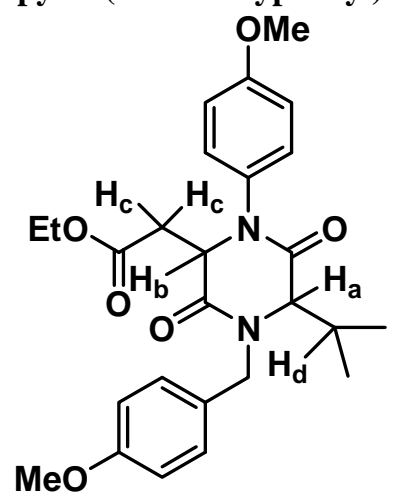

15

${ }^{1} \mathrm{H}$ NMR (400 MHz, $\left.\mathrm{CDCl}_{3}\right): \delta$ 7.24-7.30 (m, 2H, aryl), 7.0-7.06 (m, 2H, aryl), 6.85-6.95 $(\mathrm{m}, 4 \mathrm{H}, \operatorname{aryl}), 5.48(\mathrm{~d}, 1 \mathrm{H}, J=14.6 \mathrm{~Hz}, \mathrm{Bn}), 4.78\left(\mathrm{t}, 1 \mathrm{H}, J=4.5 \mathrm{~Hz}, \mathrm{H}_{\mathrm{b}}\right), 4.04(\mathrm{q}, 2 \mathrm{H}, J=$ $\left.7.3 \mathrm{~Hz},-\mathrm{OCH}_{2} \mathrm{CH}_{3}\right), 3.97(\mathrm{~d}, 1 \mathrm{H}, J=15.4 \mathrm{~Hz}, \mathrm{Bn}), 3.89\left(\mathrm{~d}, 1 \mathrm{H}, J=4.1 \mathrm{~Hz}, \mathrm{H}_{\mathrm{a}}\right), 3.81(\mathrm{~s}$, $\left.3 \mathrm{H}, \mathrm{OCH}_{3}\right), 3.80\left(\mathrm{~s}, 3 \mathrm{H}, \mathrm{OCH}_{3}\right), 3.02\left(\mathrm{dd}, 1 \mathrm{H}, J=3.5,17.4 \mathrm{~Hz}, \mathrm{H}_{\mathrm{c}}\right), 2.63(\mathrm{dd}, 1 \mathrm{H}, J=$ $\left.4.9,17 \mathrm{~Hz}, \mathrm{H}_{\mathrm{c}}\right), 2.35-2.42\left(\mathrm{~m}, 1 \mathrm{H}, \mathrm{H}_{\mathrm{d}}\right), 1.16\left(\mathrm{t}, 1 \mathrm{H}, J=7.3 \mathrm{~Hz}, \mathrm{CH}_{3}\right), 1.11(\mathrm{~d}, 3 \mathrm{H}, J=6.5$ $\left.\mathrm{Hz}, \mathrm{CHCH}_{3}\right), 1.08\left(\mathrm{~d}, 3 \mathrm{H}, J=6.5 \mathrm{~Hz}, \mathrm{CHCH}_{3}\right)$.

${ }^{13} \mathrm{C}$ NMR (100 MHz, $\left.\mathrm{CD}_{2} \mathrm{Cl} 2\right): \delta 170.3,166.0,165.9,159.7,159.5,131.2,130.0,129.4$, $128.1,114.8,114.4,64.7,60.8,57.7,55.8,55.5,47.9,35.9,32.5,20.2,17.6,14.2$.

HRMS: EIMS $\left(\mathrm{M}^{+}\right)$calcd for $\mathrm{C}_{26} \mathrm{H}_{32} \mathrm{~N}_{2} \mathrm{O}_{6} 468.2260$, found 468.2273.

$\underline{{ }^{1} \text { H-NMR (Compound 15) }}$

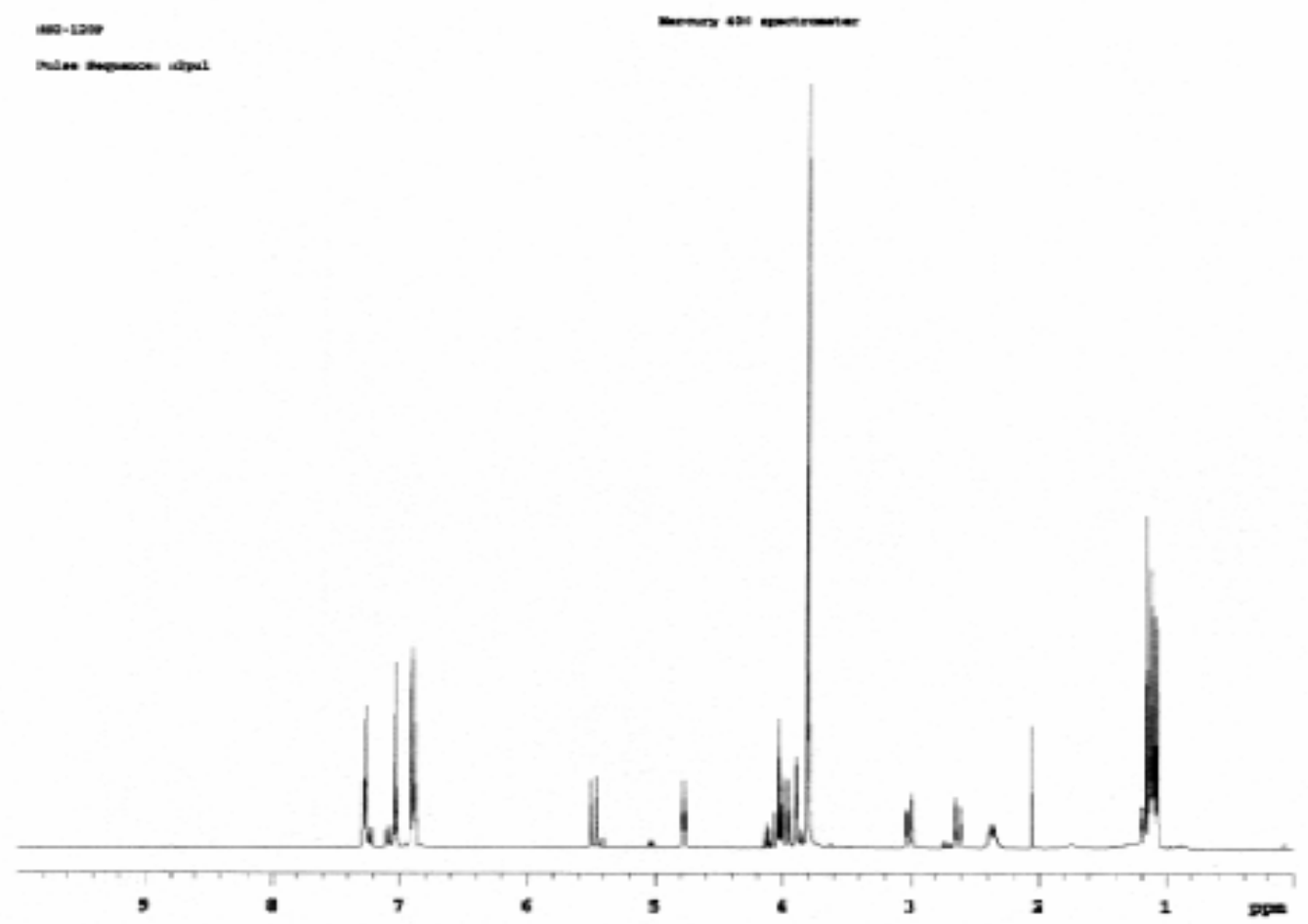




\section{${ }^{13}$ C-NMR (Compound 15)}
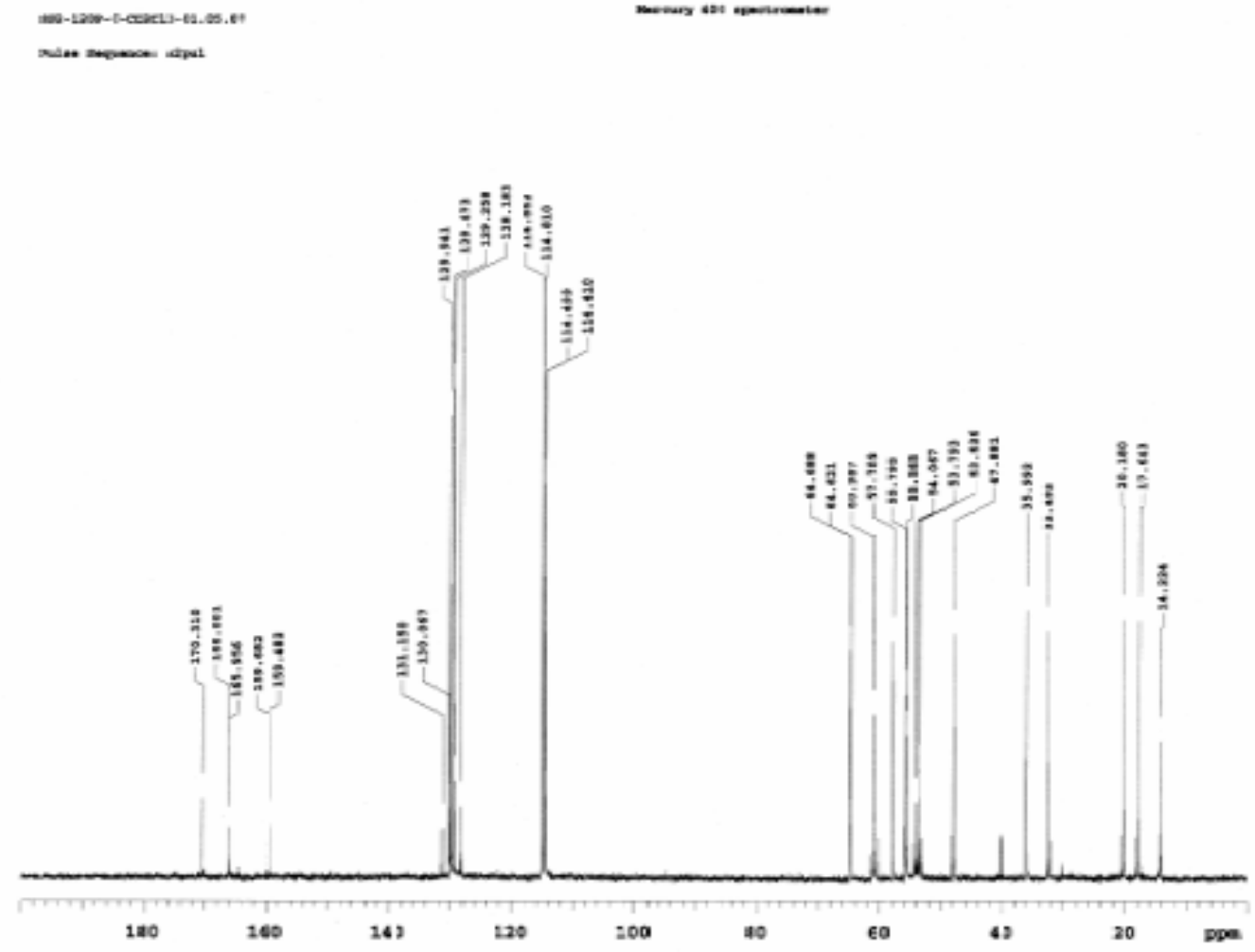

(E)-ethyl 4-((4-methoxybenzyl)(1-(4-methoxyphenylamino)-3-methyl-1-oxobutan-2-yl)amino)-4oxobut-2-enoate (16)<smiles>CCOC(=O)/C=C/C(=O)N(Cc1ccc(OC)cc1)C(C)(C(=O)Nc1ccc(OC)cc1)C(C)(C)C</smiles>

16

${ }^{1} \mathrm{H}$ NMR (400 MHz, $\left.\mathrm{CDCl}_{3}\right): \delta 8.79$ (bs, $\left.1 \mathrm{H}, \underline{\mathrm{N}}\right), 7.33$ (d, $\left.1 \mathrm{H}, J=15.4 \mathrm{~Hz},-\mathrm{C} \underline{\mathrm{H}}=\mathrm{CH}-\right)$, $7.27(\mathrm{~d}, 2 \mathrm{H}, J=8.9 \mathrm{~Hz}$, aryl), $7.05(\mathrm{~d}, 2 \mathrm{H}, J=8.1 \mathrm{~Hz}$, aryl), 6.67-6.86 (m, 5H, aryl and $\mathrm{CH}=\mathrm{CH}-$ overlap), 4.68 (bs, $2 \mathrm{H}, \mathrm{Bn}), 4.47$ (bs, $\left.1 \mathrm{H}, \mathrm{H}_{\mathrm{a}}\right), 4.16(\mathrm{q}, 2 \mathrm{H}, J=7.3 \mathrm{~Hz}$, $\left.\mathrm{OCH}_{2} \mathrm{CH}_{3}\right), 3.73\left(\mathrm{~s}, 3 \mathrm{H},-\mathrm{OC}_{3}\right), 3.69$ (s, 3H, $\left.-\mathrm{OC}_{3}\right), 2.47-2.68(\mathrm{~m}, 1 \mathrm{H}, i-\mathrm{Pr}), 1.24(\mathrm{t}$, $\left.3 \mathrm{H}, J=7.3 \mathrm{~Hz},-\mathrm{OCH}_{2} \underline{\mathrm{CH}}_{3}\right), 0.99$ (d, $\left.3 \mathrm{H}, J=6.5 \mathrm{~Hz}, i-\mathrm{Pr}\right), 0.82$ (d, $3 \mathrm{H}, J=6.5 \mathrm{~Hz}, i-\mathrm{Pr}$ ). 
${ }^{13} \mathrm{C}$ NMR $\left(100 \mathrm{MHz} \mathrm{CDCl}_{3}\right): \delta 167.6,166.9,165.1,158.9,156.2,133.8,132.4,130.8$, $128.3,128.1,121.6,114.1,113.9,67.9,61.1,55.3,55.1,49.9,26.8,19.8,18.9,14.0$.

HRMS: EIMS $\left(\mathrm{M}^{+}\right)$calcd for $\mathrm{C}_{26} \mathrm{H}_{32} \mathrm{~N}_{2} \mathrm{O}_{6} 468.2274$, found 468.2273.

\section{$\underline{{ }^{1} \text { H-NMR (Compound 16) }}$}
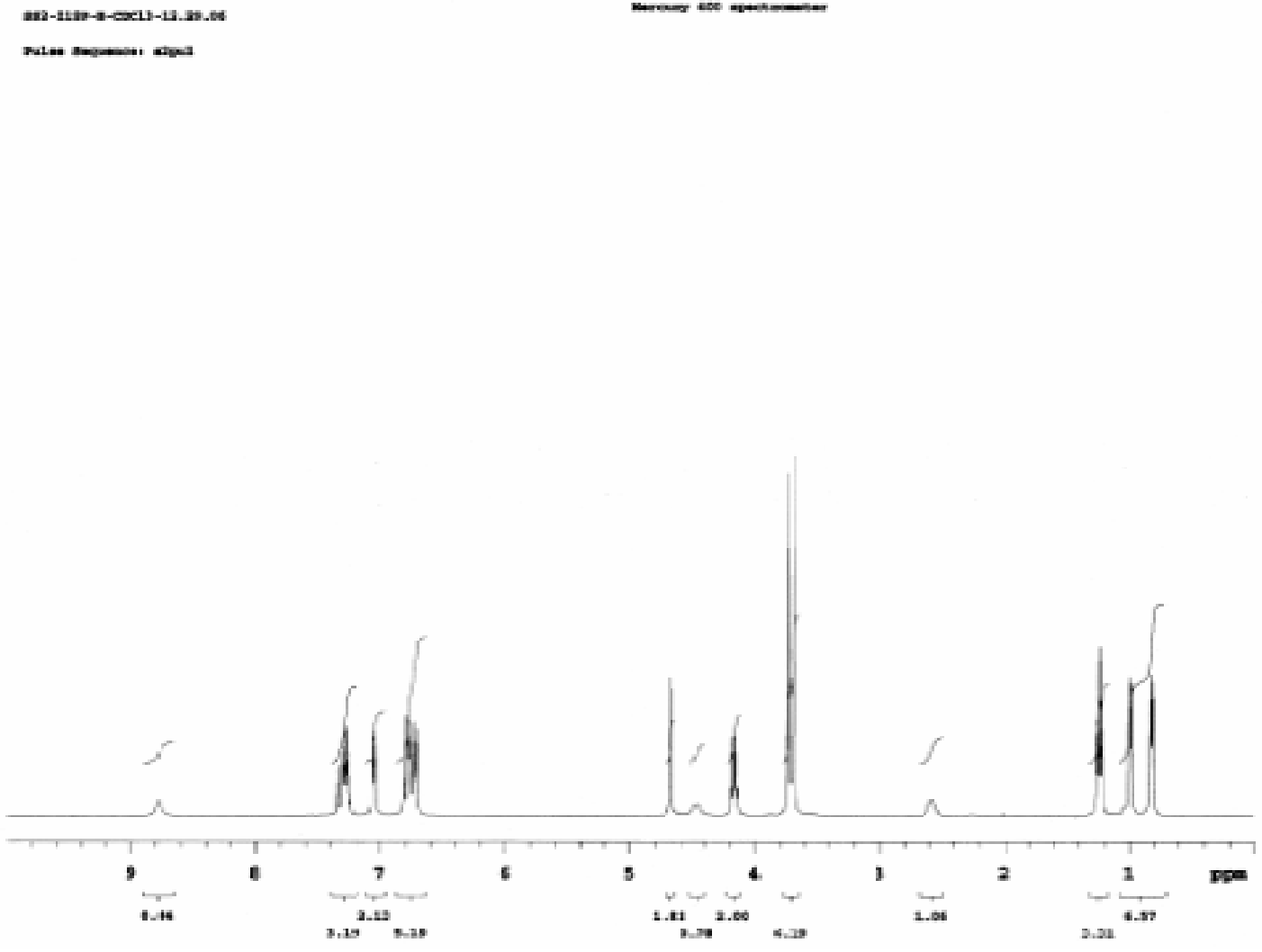

${ }^{13}$ C-NMR (Compound 16)

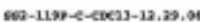

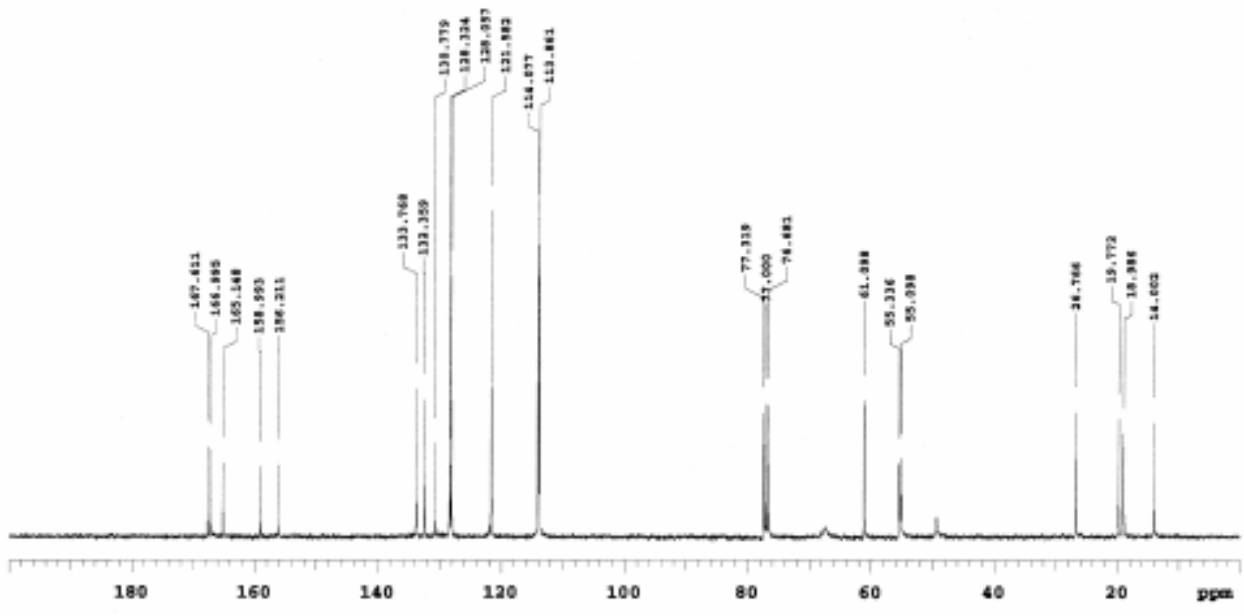


1,4-dibenzyl-3-tert-butyl-6-(2-oxo-2-phenylethyl)piperazine-2,5-dione (17)

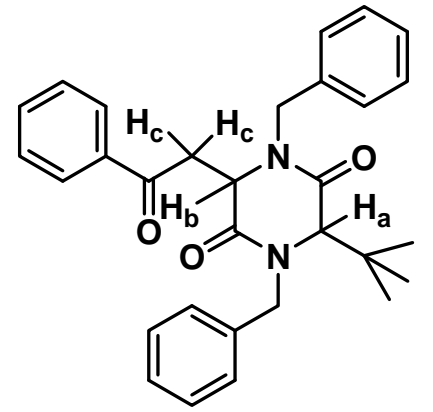

17

${ }^{1} \mathrm{H}$ NMR (400 MHz, $\mathrm{CDCl}_{3}$ ) : $\delta$ 7.8-7.89 (m, 2H, aryl), 7.52-7.61 (m, 1H, aryl), 7.05-7.49 (m, 12H, aryl), 5.65 (d, 1H, $J=14.6 \mathrm{~Hz}, \mathrm{Bn}), 4.89$ (d, 1H, $J=15.4 \mathrm{~Hz}, \mathrm{Bn}), 4.87$ (dd, 1H, $\left.J=4.1,5.7 \mathrm{~Hz}, \mathrm{H}_{\mathrm{b}}\right), 4.41$ (d, 1H, $\left.J=15.4 \mathrm{~Hz}, \mathrm{Bn}\right), 4.03$ (d, 1H, $\left.J=14.6 \mathrm{~Hz}\right), 3.86(\mathrm{~s}, 1 \mathrm{H}$, $\left.\mathrm{H}_{\mathrm{a}}\right), 3.79\left(\mathrm{dd}, 1 \mathrm{H}, J=4.9,17.8 \mathrm{~Hz}, \mathrm{H}_{\mathrm{c}}\right), 3.61\left(\mathrm{dd}, 1 \mathrm{H}, J=4.9,17.8 \mathrm{~Hz}, \mathrm{H}_{\mathrm{c}}\right), 1.18(\mathrm{~s}, 9 \mathrm{H}$, $\left.\mathrm{CH}_{3}\right)$.

${ }^{13} \mathrm{C}$ NMR $\left(100 \mathrm{MHz}, \mathrm{CDCl}_{3}\right): \delta 195.9,168.2,166.8,137.1,136.3,133.4,128.9,128.7$, $128.6,128.0,127.9,127.3,68.5,55.5,51.6,47.2,39,9,38,6,28.5$.

HRMS: EIMS $\left(\mathrm{M}^{+}\right)$calcd for $\mathrm{C}_{30} \mathrm{H}_{32} \mathrm{~N}_{2} \mathrm{O}_{3} 468.2413$, found 468.2408.

\section{${ }^{1}$ H-NMR (Compound 17)}
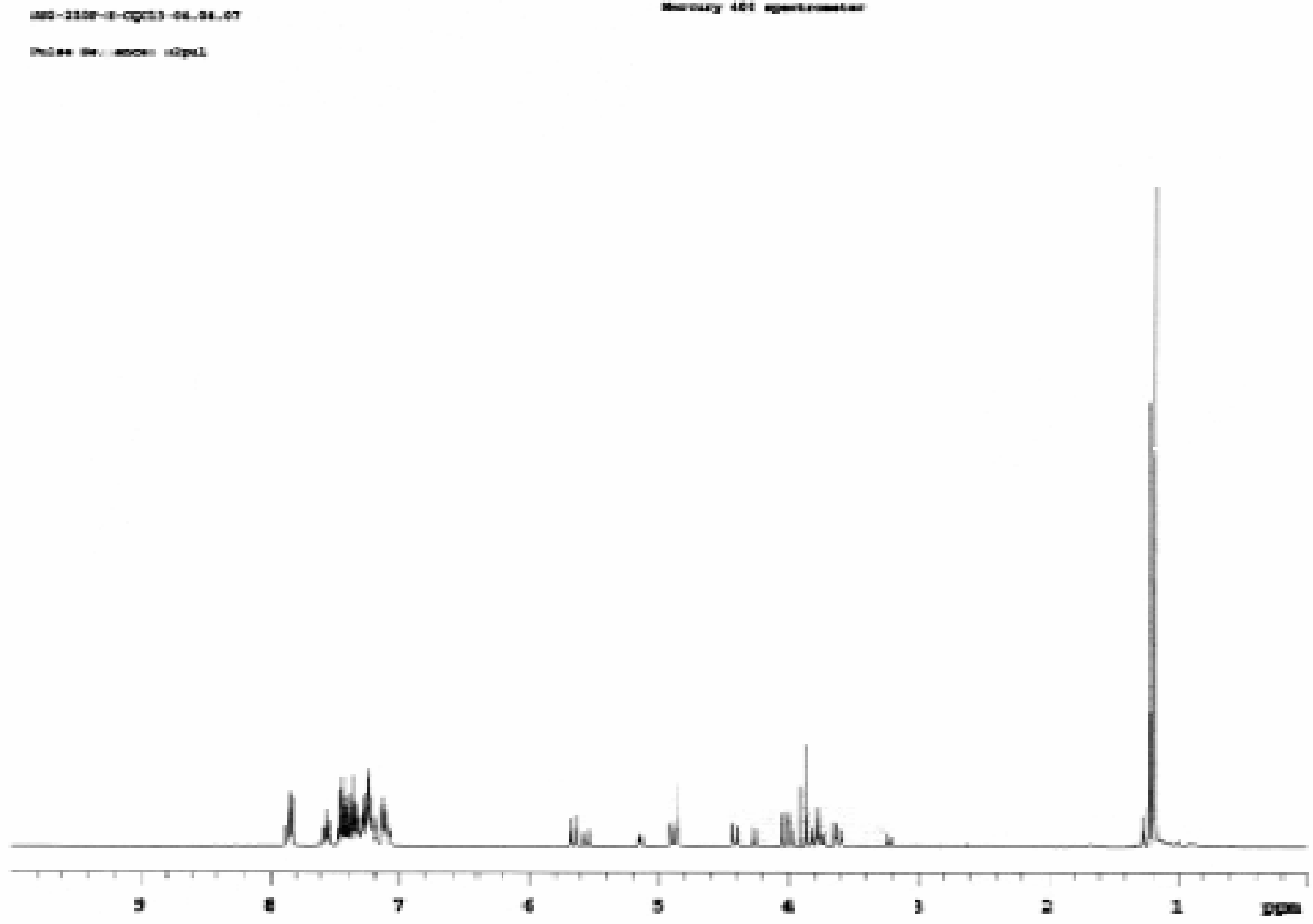


\section{${ }^{13}$ C-NMR (Compound 17)}

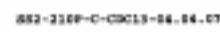
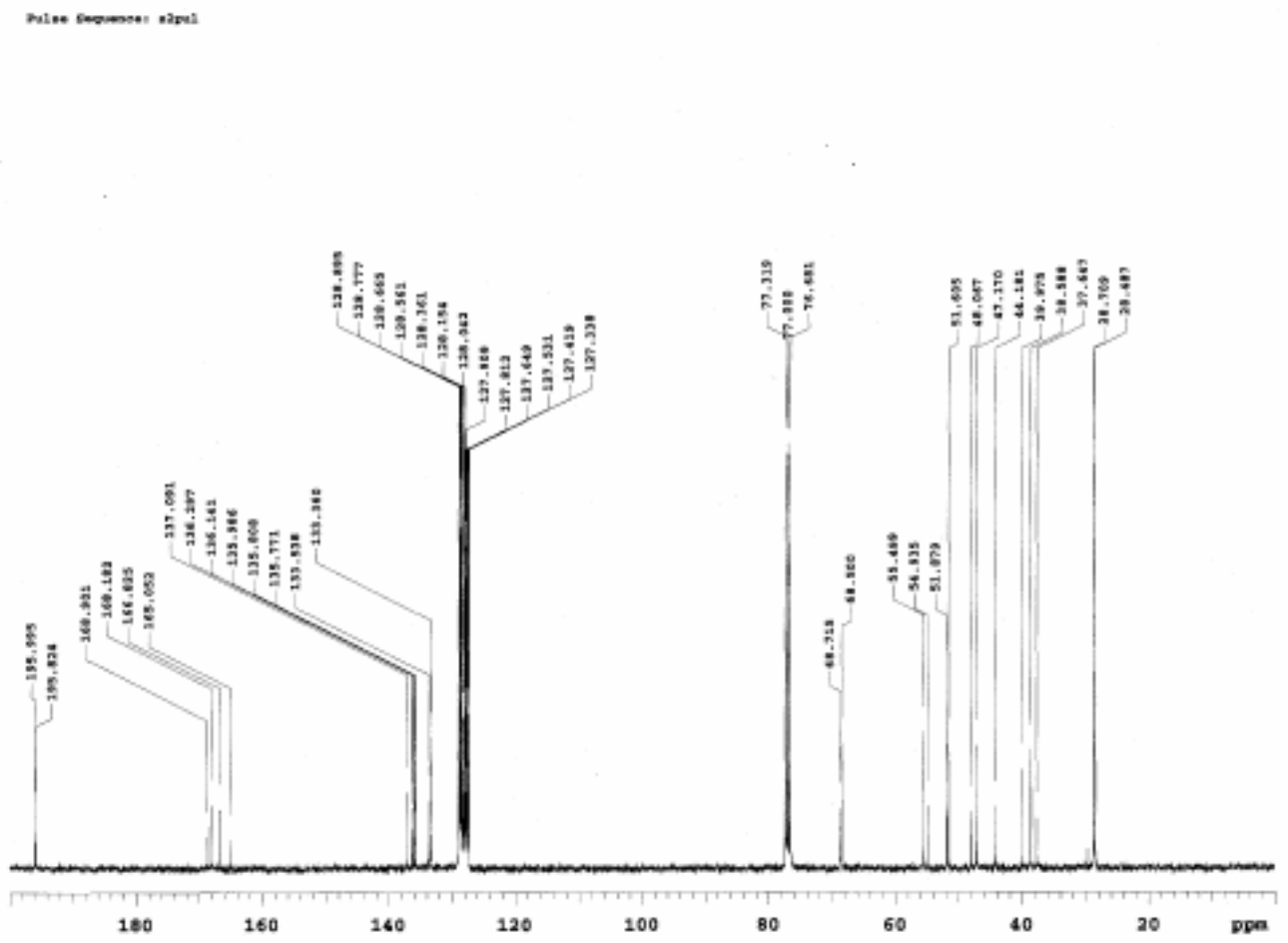

(E)-N-(1-(benzylcarbamoyl)-2,2-dimethylpropyl)-N-benzyl-4-oxo-4-phenylbut-2-enamide (18)<smiles>CC(C)(C)C(C(=O)NCc1ccccc1)N(Cc1ccccc1)C(=O)/C=C/C(=O)c1ccccc1</smiles>

18

${ }^{1} \mathrm{H}$ NMR $\left(400 \mathrm{MHz}, \mathrm{CDCl}_{3}\right): \delta$ 7.68-7.82 (m, 3H), 7.52-7.59 (m, 1H), 7.37-7.45 (m, 2H), 7.14-7.34 (m, 9H), 7.07-7.12 (m, 2H), $5.40(\mathrm{~d}, 1 \mathrm{H}, J=17.8 \mathrm{~Hz}), 5.15(\mathrm{bs}, 1 \mathrm{H}), 4.85(\mathrm{~d}$, $1 \mathrm{H}, J=17.8 \mathrm{~Hz}), 4.37(\mathrm{dd}, 1 \mathrm{H}, J=5.7,14.6 \mathrm{~Hz}), 4.15(\mathrm{dd}, 1 \mathrm{H}, J=5.7,14.6 \mathrm{~Hz}), 1.14$ (s, $9 \mathrm{H})$.

${ }^{13} \mathrm{C}$ NMR $\left(100 \mathrm{MHz}, \mathrm{CDCl}_{3}\right): \delta 189.9,168.6,167.7,138.3,137.6,136.7,134.9,133.5$, $132.9,128.7,128.6,128.0,127.6,127.2,125.6,43.5,36.7,27.6$.

HRMS: EIMS ( $\mathrm{M}^{+}$) calcd for $\mathrm{C}_{30} \mathrm{H}_{32} \mathrm{~N}_{2} \mathrm{O}_{3} 468.2413$, found 468.2411.

$\underline{{ }^{1} \text { H-NMR (Compound 18) }}$ 


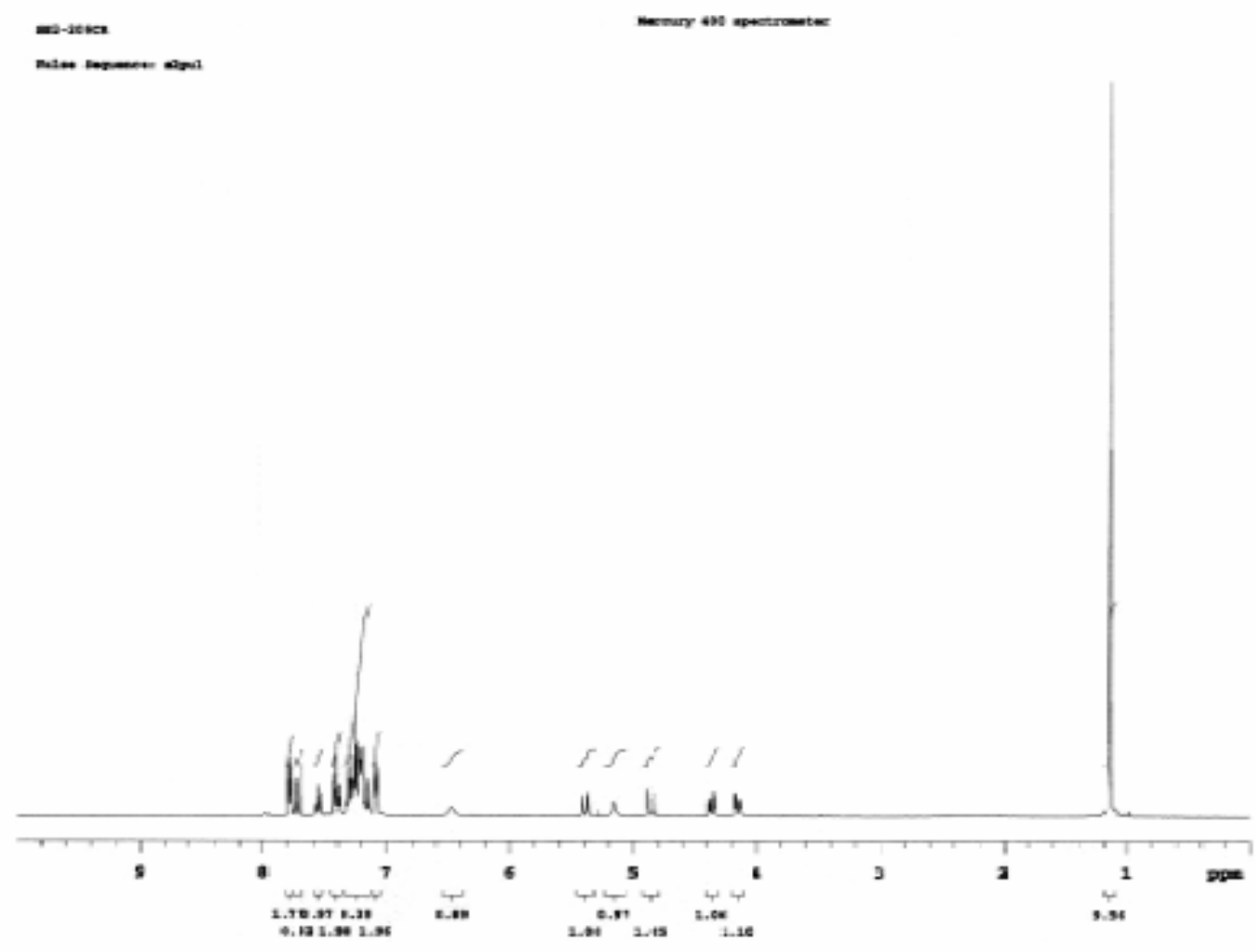

${ }^{13}$ C-NMR (Compound 18)

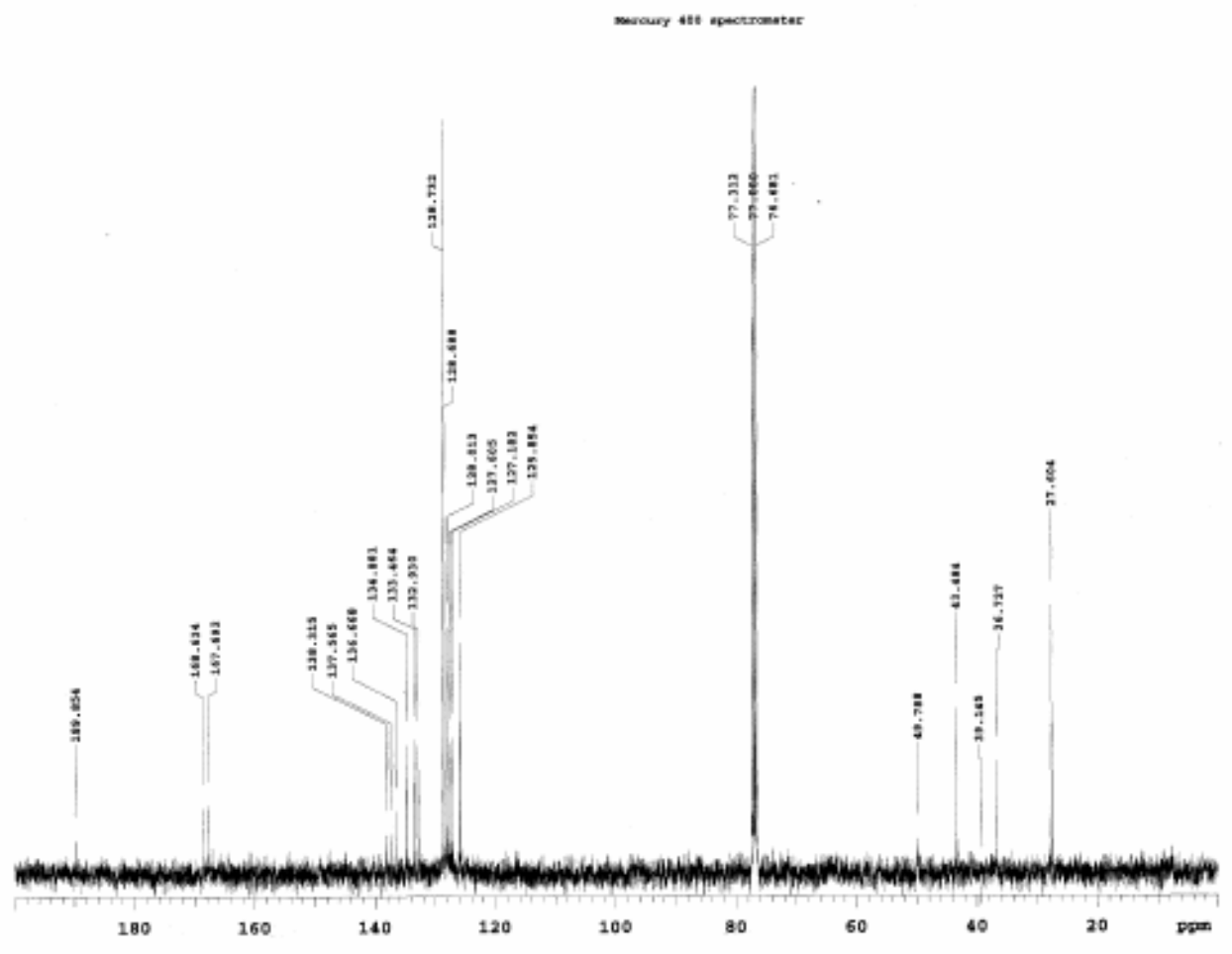


Ethyl 2-(4-(4-methoxybenzyl)-5-tert-butyl-1-butyl-3,6-dioxopiperazin-2-yl)acetate (19) ${ }^{\mathrm{c}}$

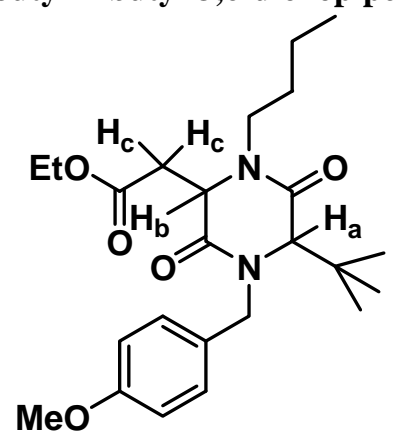

19

${ }^{1} \mathrm{H}$ NMR $\left(500 \mathrm{MHz}, \mathrm{CDCl}_{3}\right): \delta 7.15(\mathrm{~d}, 2 \mathrm{H}, J=8.9 \mathrm{~Hz}$, aryl), $6.83(\mathrm{~d}, 2 \mathrm{H}, J=8.6 \mathrm{~Hz}$, aryl), $5.57(\mathrm{~d}, 1 \mathrm{H}, J=14.9 \mathrm{~Hz}, \mathrm{Bn}), 4.54\left(\mathrm{dd}, 1 \mathrm{H}, J=4.3,5.8 \mathrm{~Hz}, \mathrm{H}_{\mathrm{b}}\right), 4.08-4.21(\mathrm{~m}, 2 \mathrm{H}$, $\left.-\mathrm{OCH}_{2} \mathrm{CH}_{3}\right), 3.93-4.03(\mathrm{~m}, 1 \mathrm{H}, n-\mathrm{Bu}), 3.88(\mathrm{~d}, 1 \mathrm{H}, J=14.9 \mathrm{~Hz}, \mathrm{Bn}), 3.77\left(\mathrm{~s}, 3 \mathrm{H}, \mathrm{OCH}_{3}\right)$, $3.65\left(\mathrm{~s}, 1 \mathrm{H}, \mathrm{H}_{\mathrm{a}}\right), 3.17\left(\mathrm{dd}, 1 \mathrm{H}, J=4.1,16.9 \mathrm{~Hz}, \mathrm{H}_{\mathrm{c}}\right), 3.01\left(\mathrm{dd}, 1 \mathrm{H}, J=6.1,16.9 \mathrm{~Hz}, \mathrm{H}_{\mathrm{c}}\right)$, 2.78-2.87 (m, $1 \mathrm{H}, n-\mathrm{Bu}), 1.26-1.56(\mathrm{~m}, 4 \mathrm{H}, n-\mathrm{Bu}), 1.23\left(\mathrm{t}, 3 \mathrm{H}, J=7.3 \mathrm{~Hz},-\mathrm{OCH}_{2} \mathrm{CH}_{3}\right)$, 1.09 (s, 9H, t-Bu), 0.92 (t, 3H, $\left.J=7.3 \mathrm{~Hz},-\mathrm{CH}_{2} \mathrm{CH}_{2} \mathrm{CH}_{2} \mathrm{CH}_{3}\right)$.

${ }^{13} \mathrm{C}$ NMR $\left(125 \mathrm{MHz}, \mathrm{CDCl}_{3}\right): \delta 170.1,167.5,165.9,159.2,129.4,127.6,114.2,67.6$, $60.9,55.2,55.1,50.9,42.9,39.9,35.1,29.4,20.3,14.1,13.7$.

HRMS: EIMS $\left(\mathrm{M}^{+}\right)$calcd for $\mathrm{C}_{24} \mathrm{H}_{36} \mathrm{~N}_{2} \mathrm{O}_{5}$ 432.2624, found 432.2625.

${ }^{1}$ H-NMR (Compound 19)

(c) NOe data obtained from 19 corresponds well with published data indicating a trans relationship between napthal and methylene ethyl ester. See: Davies, S. G.; Garner, C.; Ouzman, J. V. A.; Roberts, P. M.; Smith, A. D.; Snow, E. J.; Thomson, J. E.; Tamayo, J. A.; Vickers, R. J. Org. Biomol. Chem., 2007, 5, 2138-2147. 


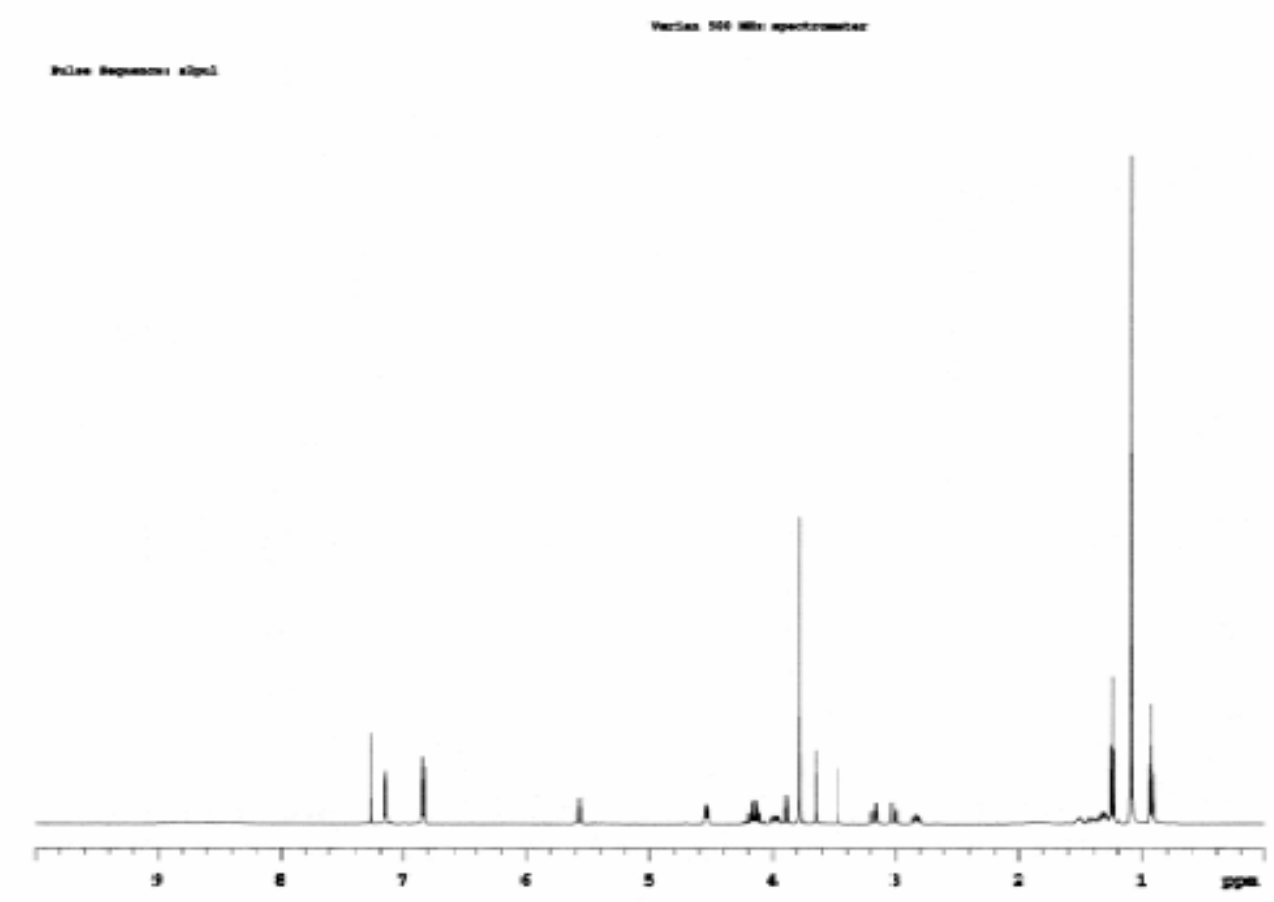

\section{${ }^{13}$ C-NMR (Compound 19)}
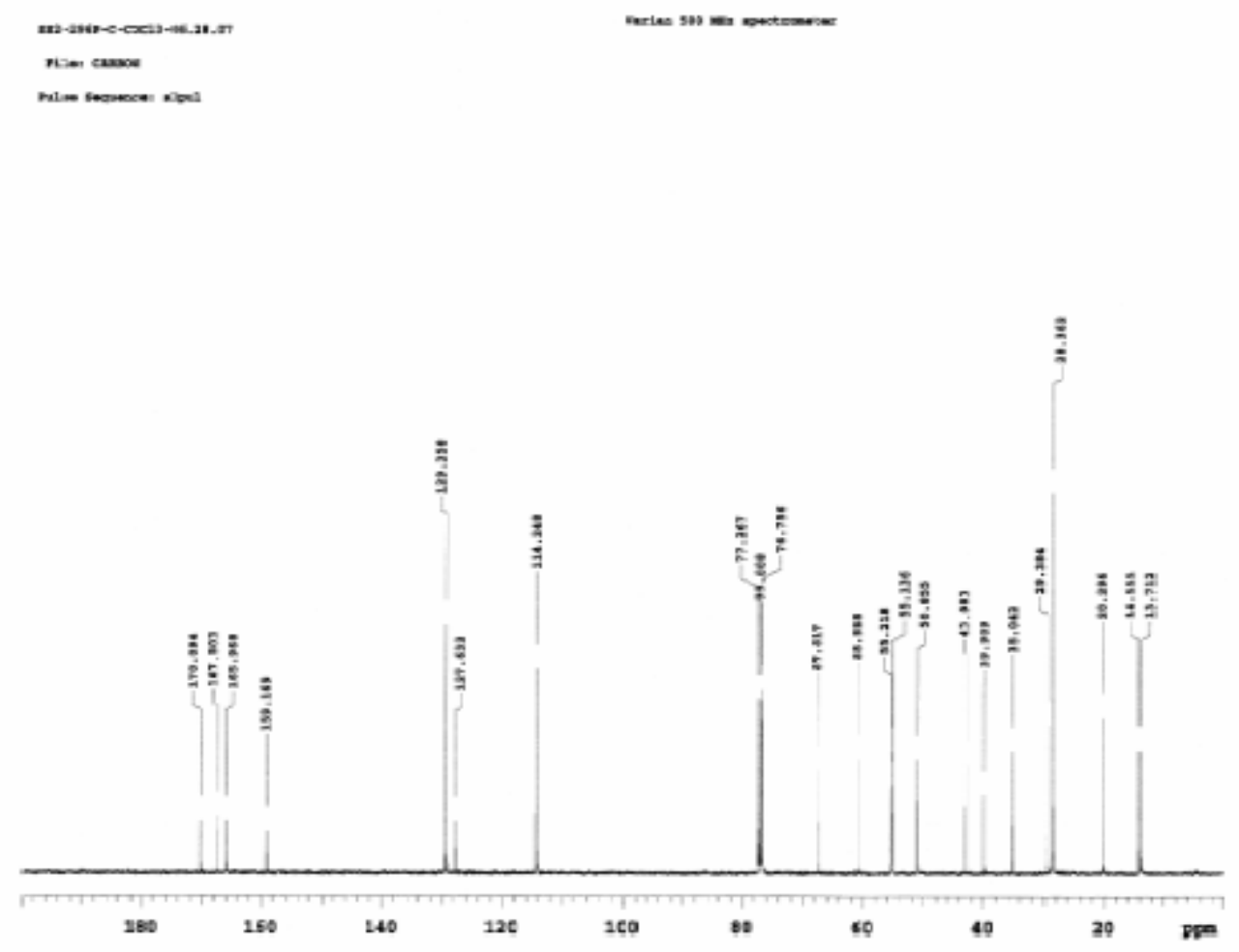
(E)-ethyl 4-((1-(butylamino)-3,3-dimethyl-1-oxobutan-2-yl)(4-methoxybenzyl)amino)-4-oxobut-2enoate (20)

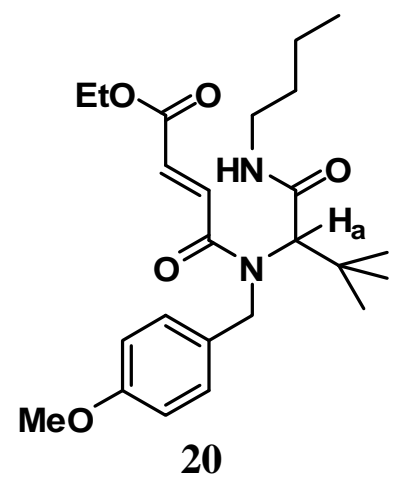

${ }^{1} \mathrm{H}$ NMR (300 MHz, $\left.\mathrm{CDCl}_{3}\right): \delta 7.20$ (d, 1H, J=15.3 Hz, $\left.-\mathrm{CH}=\mathrm{CH}-\right), 6.99$ (d, 2H, $J=8.7$

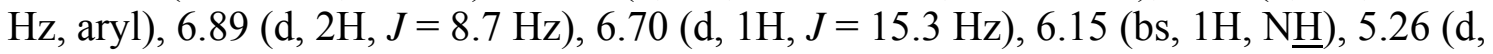
$1 \mathrm{H}, J=17.1, \mathrm{Bn}), 5.01\left(\mathrm{bs}, 1 \mathrm{H}, \mathrm{H}_{\mathrm{a}}\right), 4.72(\mathrm{~d}, 1 \mathrm{H}, J=17.4 \mathrm{~Hz}, \mathrm{Bn}), 4.11-4.19(\mathrm{~m}, 2 \mathrm{H}$, -

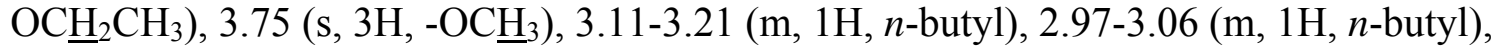
1.27-1.43 (m, 4H, n-butyl), 1.24 (t, 3H, $\left.J=7.3 \mathrm{~Hz},-\mathrm{OCH}_{2} \mathrm{C}_{3}\right), 1.08$ (s, 9H, t-butyl), $0.88(\mathrm{t}, 3 \mathrm{H}, J=7.3 \mathrm{~Hz}, n$-butyl).

${ }^{13} \mathrm{C}$ NMR (75 MHz, $\left.\mathrm{CDCl}_{3}\right): \delta 168.8,167.3,165.4,158.7,134.3,131.4,127.2,114.1$, $60.9,55.2,39.1,36.6,31.3,28.4,27.6,20.1,14.1,13.7$.

HRMS: EIMS $\left(\mathrm{M}^{+}\right)$calcd for $\mathrm{C}_{24} \mathrm{H}_{36} \mathrm{~N}_{2} \mathrm{O}_{5} 432.2624$, found 432.2620

\section{${ }^{1}$ H-NMR (Compound 20)}

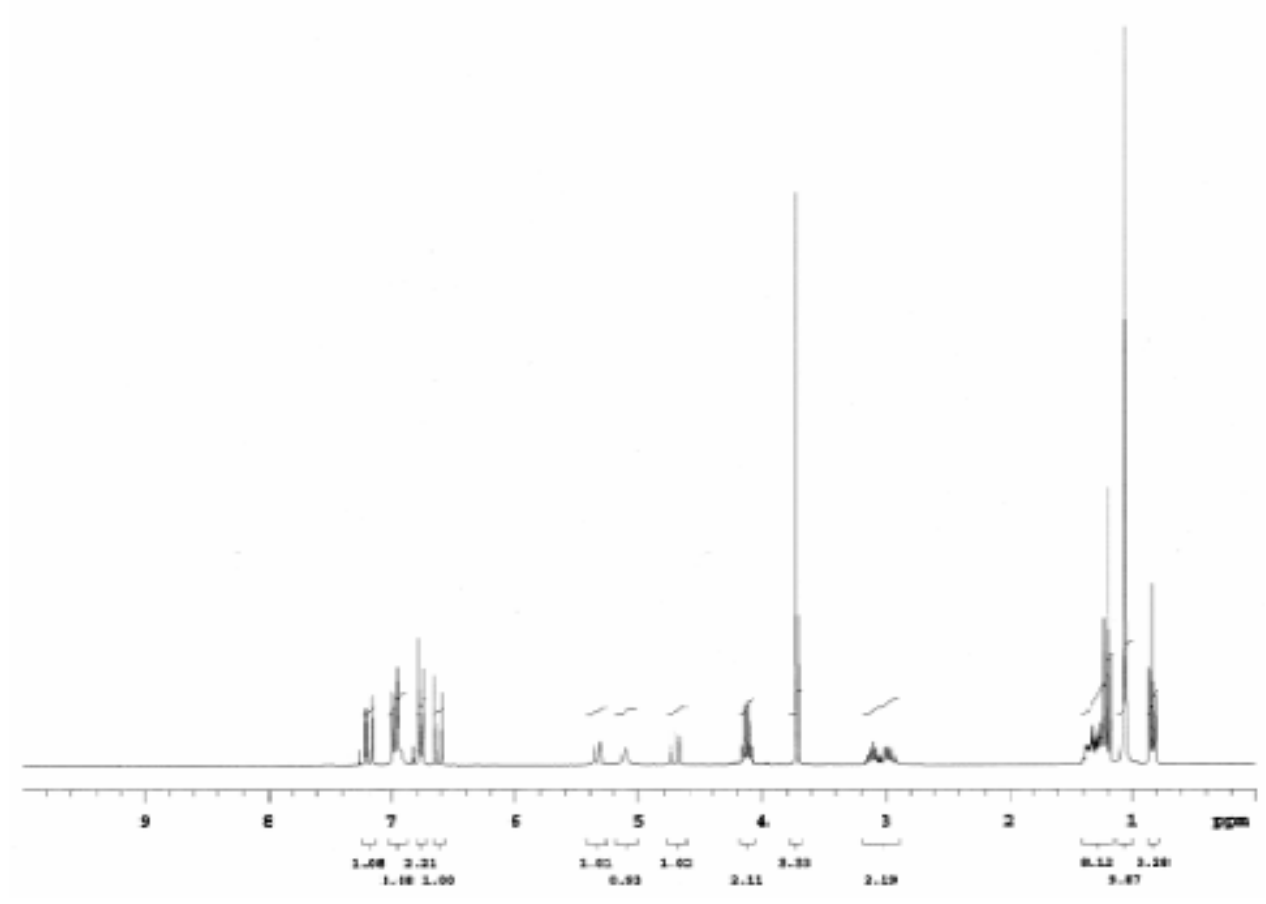




\section{${ }^{13}$ C-NMR (Compound 20)}

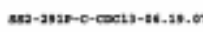
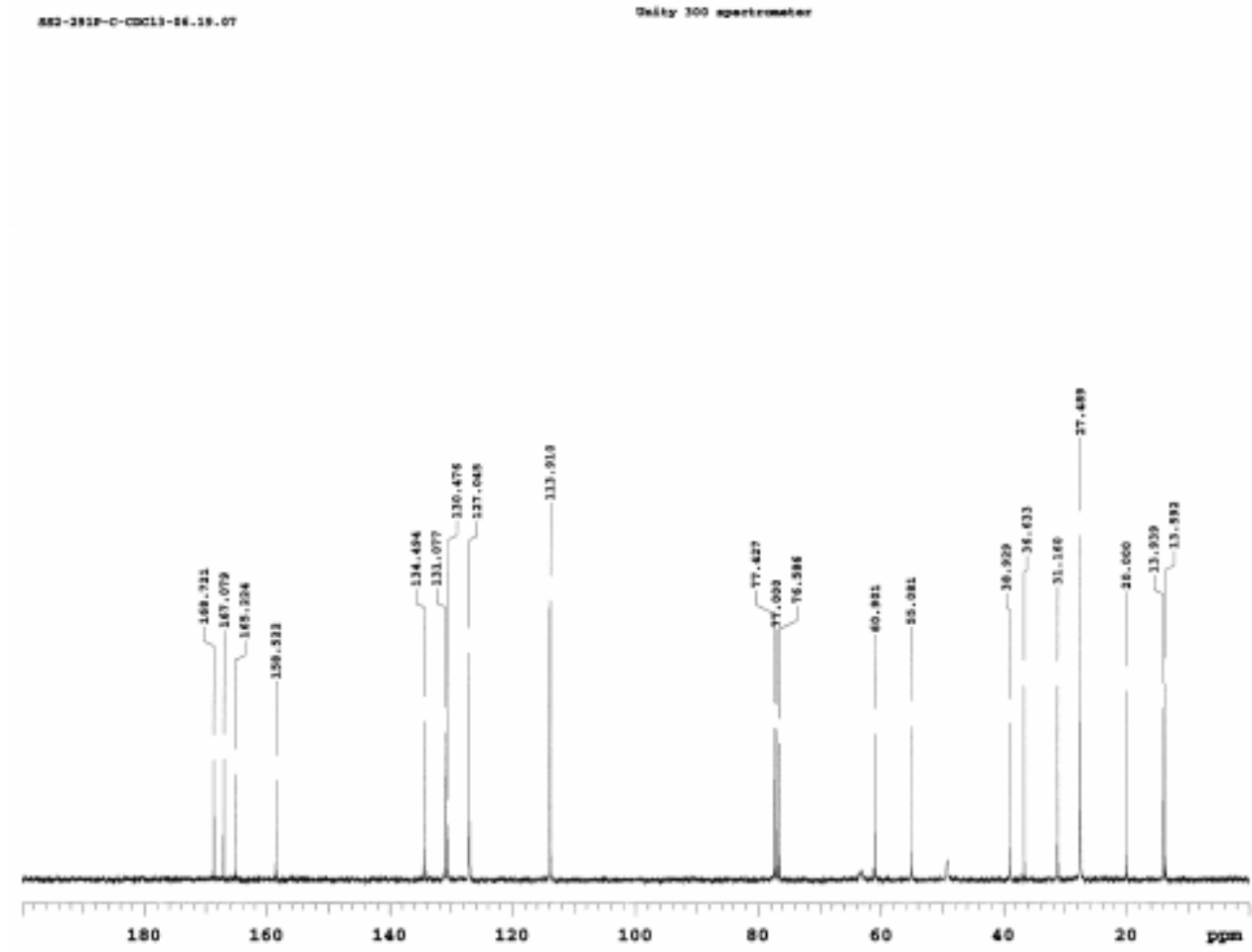

Ethyl 2-(4-(4-hydroxybenzyl)-5-tert-butyl-1-benzyl-3,6-dioxopiperazin-2-yl)acetate (21)

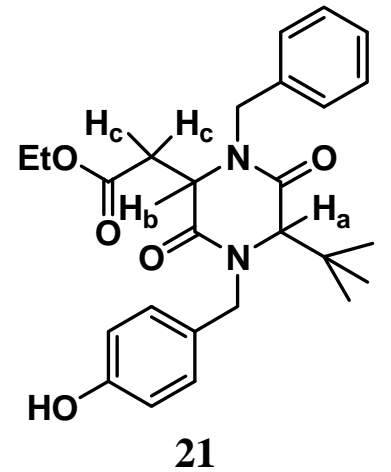

${ }^{1} \mathrm{H}$ NMR $\left(500 \mathrm{MHz}, \mathrm{CDCl}_{3}\right): \delta 7.56$ (bs, $\left.1 \mathrm{H},-\mathrm{OH}\right), 7.24-7.37$ (m, 3H, aryl), 7.20 (m, 2H, aryl), $7.08(\mathrm{~d}, 2 \mathrm{H}, J=8.1 \mathrm{~Hz}$, aryl), $6.63(\mathrm{~d}, 2 \mathrm{H}, J=8.6 \mathrm{~Hz}), 5.56(\mathrm{~d}, 1 \mathrm{H}, J=15.2 \mathrm{~Hz}$, $\mathrm{Bn}), 5.16(\mathrm{~d}, 1 \mathrm{H}, J=15.2 \mathrm{~Hz}, \mathrm{Bn}), 4.53\left(\mathrm{dd}, 1 \mathrm{H}, J=4.1,5.6 \mathrm{~Hz}, \mathrm{H}_{\mathrm{b}}\right), 4.32(\mathrm{~d}, 1 \mathrm{H}, J=$ $15.7 \mathrm{~Hz}, \mathrm{Bn}), 3.98-4.16\left(\mathrm{~m}, 2 \mathrm{H},-\mathrm{OCH}_{2} \mathrm{CH}_{3}\right), 3.88(\mathrm{~d}, 1 \mathrm{H}, J=15.2 \mathrm{~Hz}, \mathrm{Bn}), 3.17$ (dd, $\left.1 \mathrm{H}, J=3.6,17.2 \mathrm{~Hz}, \mathrm{H}_{\mathrm{c}}\right), 2.97\left(\mathrm{dd}, 1 \mathrm{H}, J=6.1,17.2 \mathrm{~Hz}, \mathrm{H}_{\mathrm{c}}\right), 1.18(\mathrm{t}, 3 \mathrm{H}, J=7.3 \mathrm{~Hz},-$ $\left.\mathrm{OCH}_{2} \mathrm{C}_{3}\right), 1.12(\mathrm{~s}, 9 \mathrm{H}, t-\mathrm{Bu})$.

${ }^{13} \mathrm{C}$ NMR $\left(125 \mathrm{MHz}, \mathrm{CDCl}_{3}\right): \delta 170.1,167.7,167.5,156.4,136.1,129.5,128.9,127.8$, $127.6,126.3,115.7,67.7,61.0,55.8,51.3,47.0,40.1,34.6,28.3,14.0$.

HRMS: EIMS $\left(\mathrm{M}^{+}\right)$calcd for $\mathrm{C}_{26} \mathrm{H}_{32} \mathrm{~N}_{2} \mathrm{O}_{5} 452.2311$, found 452.2315 
$\underline{{ }^{1} \text { H-NMR (Compound 21) }}$

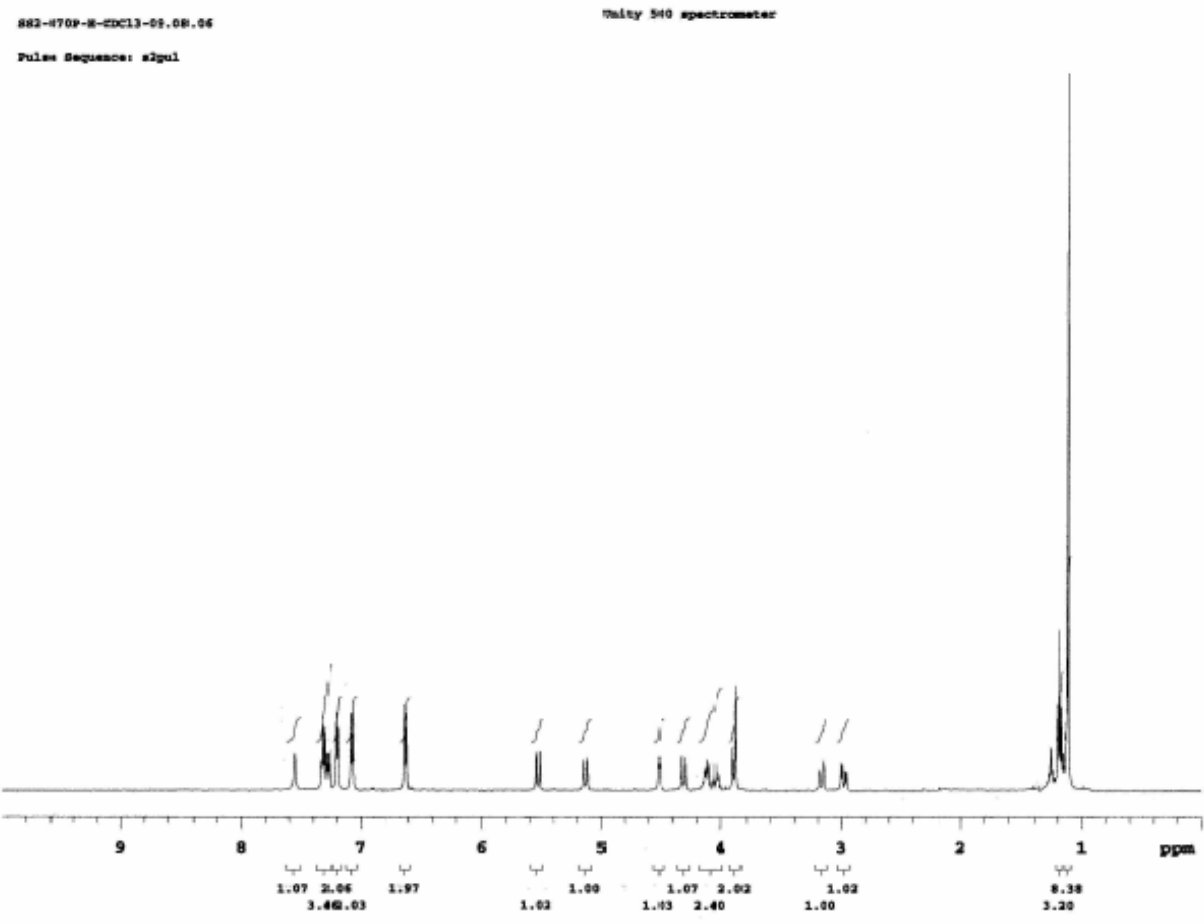

\section{${ }^{13}$ C-NMR (Compound 21)}

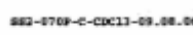

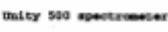

reter sequenset mapel

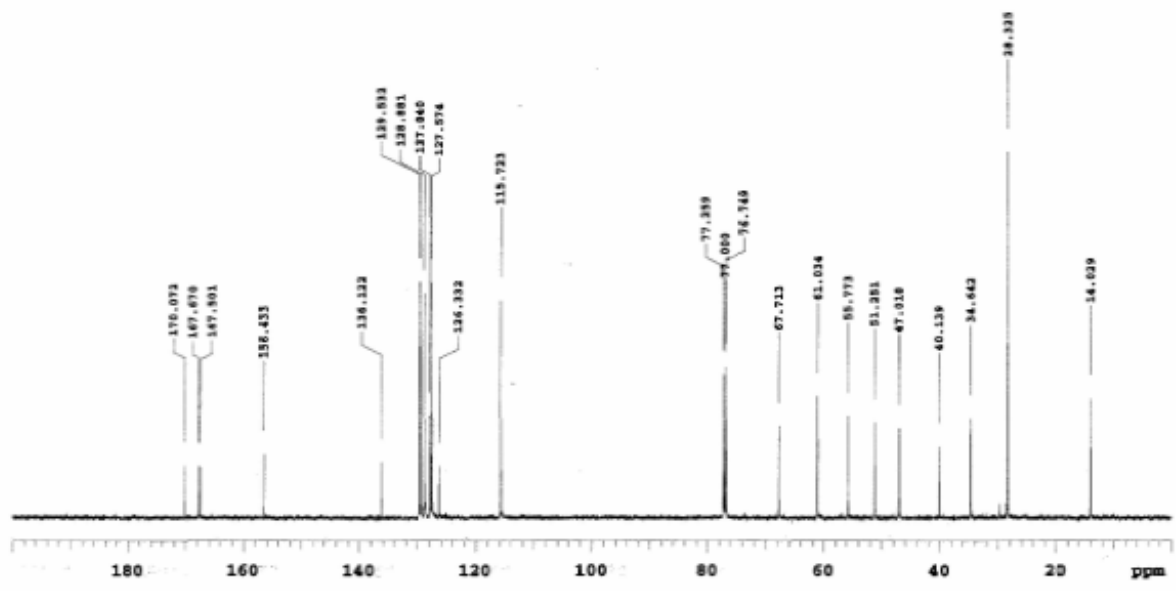


(E)-ethyl 4-((1-(benzylamino)-3,3-dimethyl-1-oxobutan-2-yl)(4-hydroxybenzyl)amino)-4-oxobut-2enoate (22)

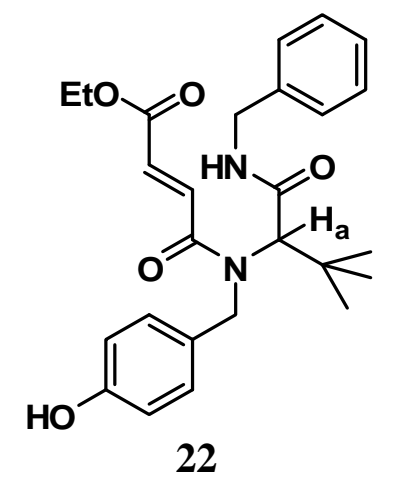

${ }^{1} \mathrm{H}$ NMR $\left(500 \mathrm{MHz}, \mathrm{CDCl}_{3}\right): \delta 7.57(\mathrm{bs}, 1 \mathrm{H},-\mathrm{N} \underline{\mathrm{H}}), 7.11-7.34(\mathrm{~m}, 6 \mathrm{H}$, aryl and $-\mathrm{C} \underline{\mathrm{H}}=\mathrm{CH}-$ overlap), $6.78(\mathrm{~d}, 2 \mathrm{H}, J=8.1 \mathrm{~Hz}$, aryl), $6.66(\mathrm{~d}, 1 \mathrm{H}, J=15.2 \mathrm{~Hz},-\mathrm{CH}=\mathrm{C} \underline{\mathrm{H}}-), 6.48$ (d, 2H, $J=8.1 \mathrm{~Hz}$, aryl), 5.34 (d. $1 \mathrm{H}, J=15.2 \mathrm{~Hz}, \mathrm{Bn}), 5.16$ (bs, $\left.1 \mathrm{H}, \mathrm{H}_{\mathrm{a}}\right), 4.66$ (d, 1H, $J=15.2$ $\mathrm{Hz}, \mathrm{Bn}), 4.37$ (dd, 1H, J=6.1, $14.7 \mathrm{~Hz}, \mathrm{Bn}), 4.14$ (m, 2H, $\left.-\mathrm{OC}_{2} \mathrm{CH}_{3}\right), 4.02$ (d, 1h, $J=$ $12.7 \mathrm{~Hz}, \mathrm{Bn}), 1.33$ (t, $\left.3 \mathrm{H}, J=7.3 \mathrm{~Hz},-\mathrm{OCH}_{2} \underline{\mathrm{CH}}_{3}\right), 1.11$ (s, 9H, t-butyl).

${ }^{13} \mathrm{C}$ NMR $\left(125 \mathrm{MHz}, \mathrm{CDCl}_{3}\right): \delta 168.8,167.6,165.4,155.4,137.3,134.1,131.7,128.6$, $127.8,127.8,127.5,126.9,115.6,61.2,43.5,36.9,27.6,13.9$.

HRMS: EIMS $\left(\mathrm{M}^{+}\right)$calcd for $\mathrm{C}_{26} \mathrm{H}_{32} \mathrm{~N}_{2} \mathrm{O}_{5}$ 452.2311, found 452.2314.

\section{$\underline{{ }^{1} \text { H-NMR (Compound 22) }}$}

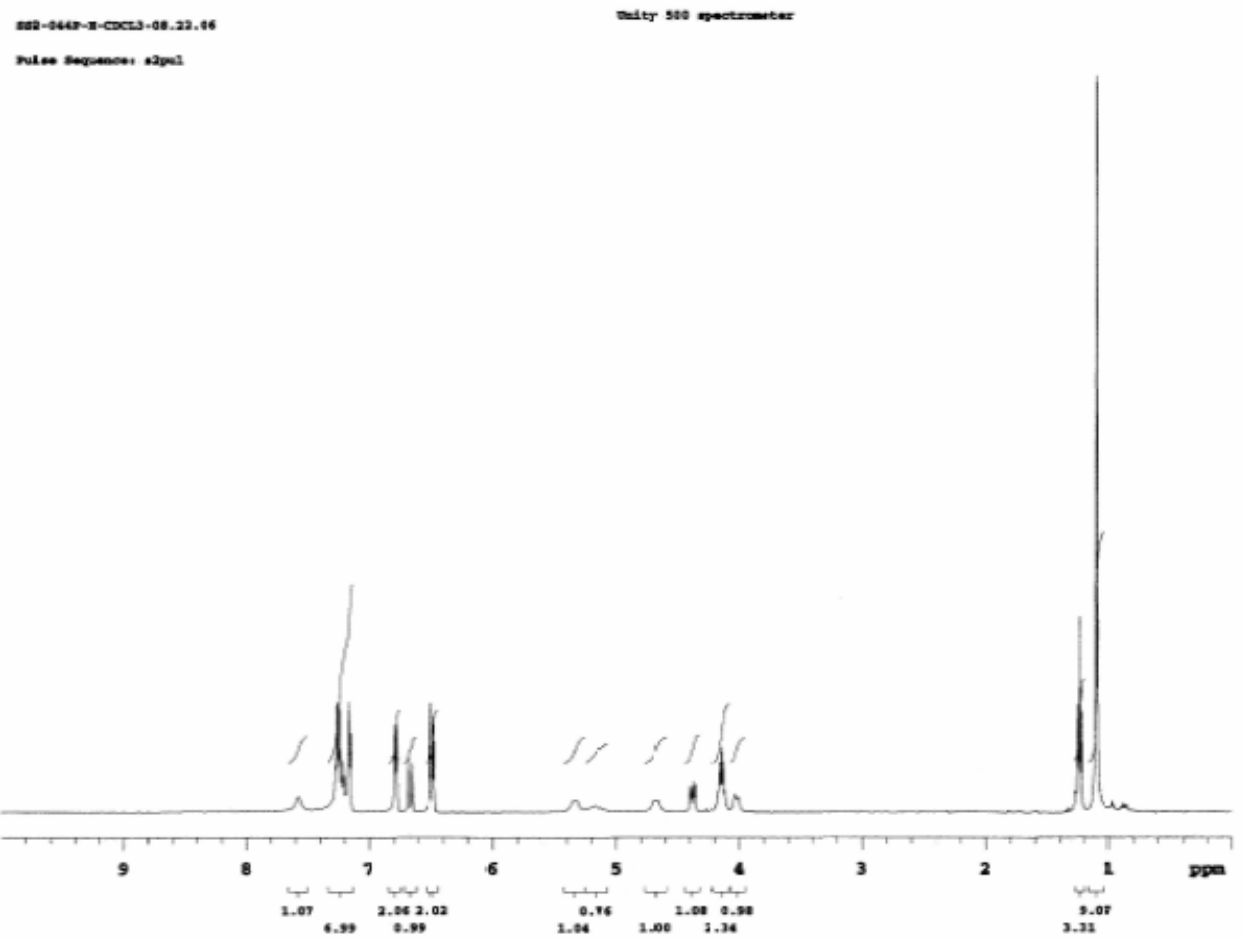




\section{${ }^{13}$ C-NMR (Compound 22)}
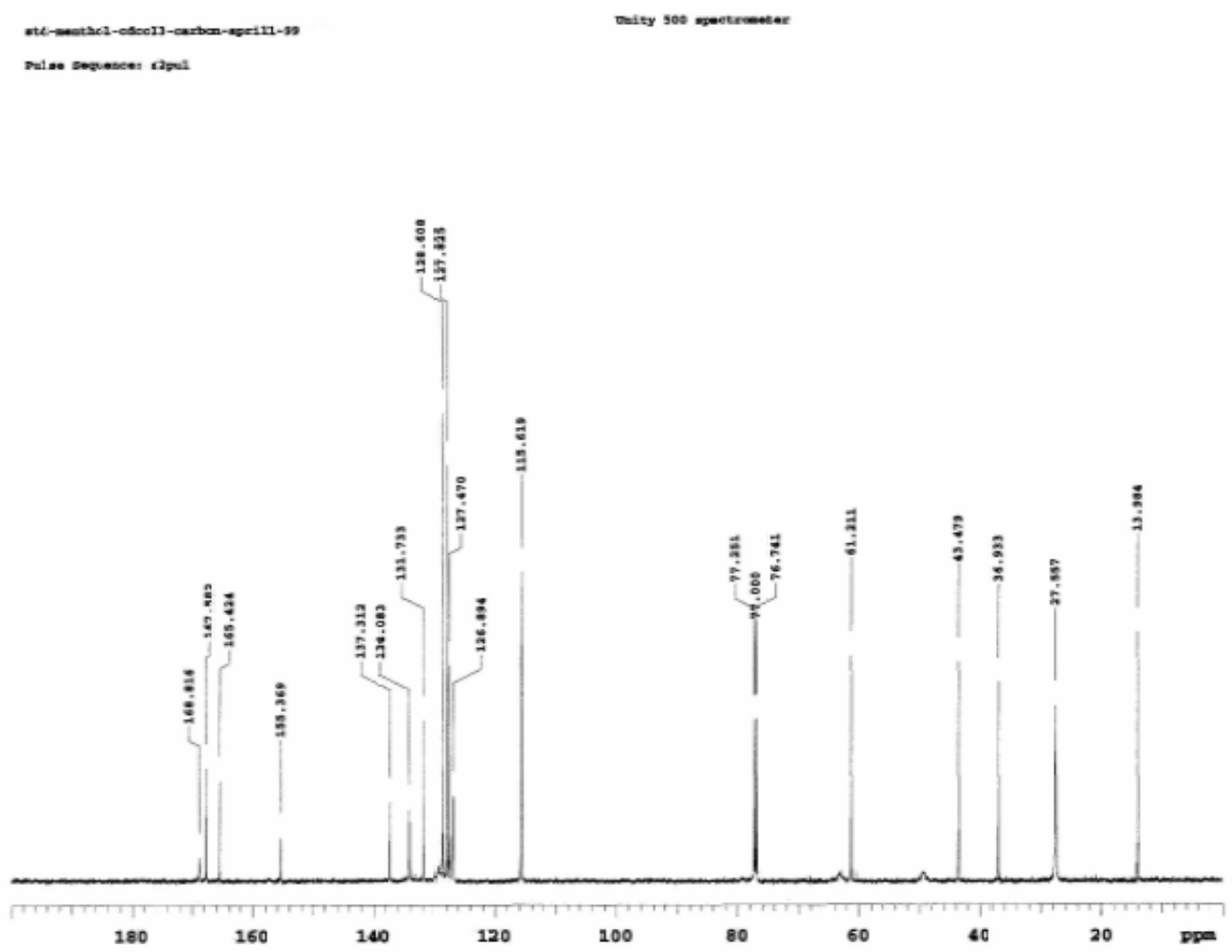

Ethyl 2-(4-(3,5-dimethoxybenzyl)-1-benzyl-3,6-dioxo-5-(thiophen-2-yl)piperazin-2-yl)acetate (23)

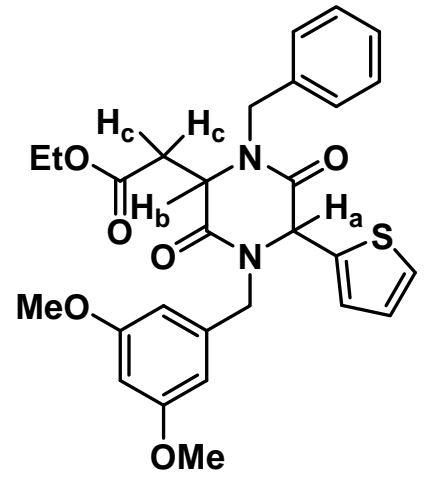

23

${ }^{1} \mathrm{H}$ NMR $\left(500 \mathrm{MHz}, \mathrm{CDCl}_{3}\right): \delta$ 7.0-7.35 $(\mathrm{m}, 8 \mathrm{H}$, aryl), 6.36-6.45 (m, 3H, aryl), $6.29(\mathrm{~s}$, $\left.1 \mathrm{H}, \mathrm{H}_{\mathrm{a}}\right), 5.55(\mathrm{~d}, 1 \mathrm{H}, J=14.9 \mathrm{~Hz}, \mathrm{Bn}), 5.32(\mathrm{~d}, 1 \mathrm{H}, J=15.6 \mathrm{~Hz}, \mathrm{Bn}), 4.22(\mathrm{dd}, 1 \mathrm{H}, J=$ 2.8, $4.6 \mathrm{~Hz}, \mathrm{H}_{\mathrm{b}}$ ), 4.02-4.15 (m, 2H, $\left.-\mathrm{OC}_{2} \mathrm{CH}_{3}\right), 3.98$ (d, $\left.1 \mathrm{H}, J=15.6 \mathrm{~Hz}, \mathrm{Bn}\right), 3.77$ (s, $6 \mathrm{H}), 3.56(\mathrm{~d}, 1 \mathrm{H}, J=14.9 \mathrm{~Hz}, \mathrm{Bn}), 3.32\left(\mathrm{dd}, 1 \mathrm{H}, J=2.8,17.5 \mathrm{~Hz}, \mathrm{H}_{\mathrm{c}}\right), 2.95(\mathrm{dd}, 1 \mathrm{H}, J=$ $4.9,17.5 \mathrm{~Hz}, \mathrm{H}_{\mathrm{c}}$ ), 1.17 (t, $3 \mathrm{H}, J=7.3 \mathrm{~Hz}, \mathrm{CH}_{3}$ ).

${ }^{13} \mathrm{C}$ NMR $\left(125 \mathrm{MHz}, \mathrm{CDCl}_{3}\right): \delta 169.5,165.1,164.9,160.9,139.8,137.1,134.9,128.9$, $127.9,127.1,126.4,106.2,100.2,60.9,58.5,55.2,54.5,47.1,46.7,34.4,13.9$.

HRMS: EIMS $\left(\mathrm{M}^{+}\right)$calcd for $\mathrm{C}_{28} \mathrm{H}_{30} \mathrm{~N}_{2} \mathrm{O}_{6} \mathrm{~S} 522.1825$, found 522.1829. 
${ }^{1}$ H-NMR (Compound 23)
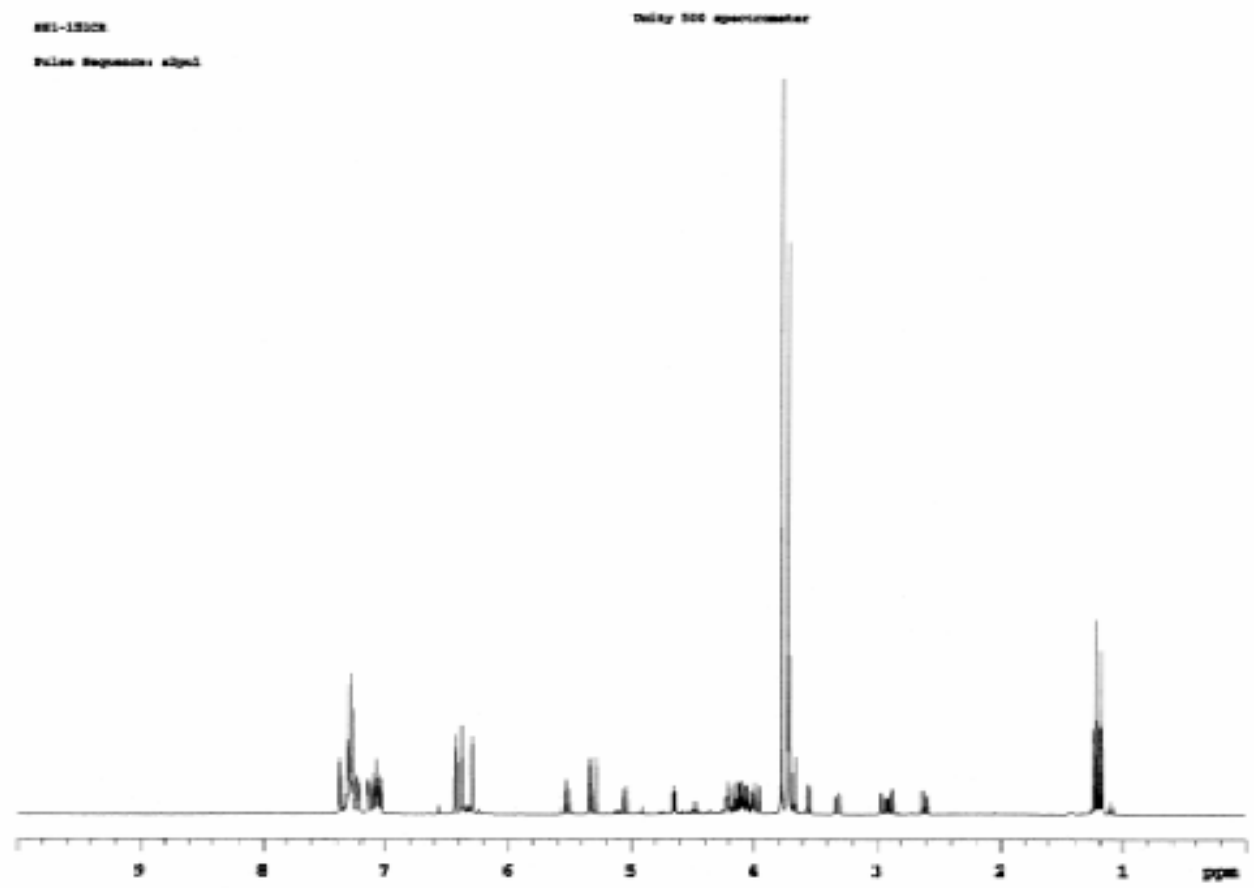

${ }^{13}$ C-NMR (Compound 23)

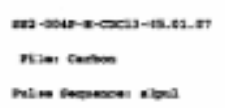

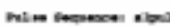

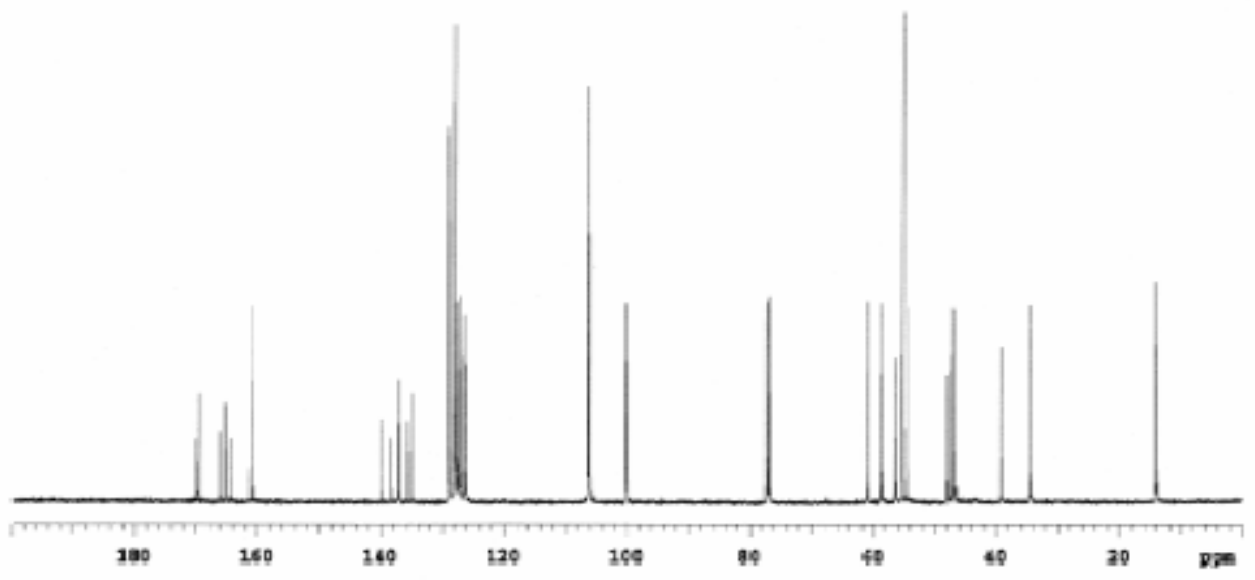


Ethyl 2-(1-benzyl-4-(4-methoxybenzyl)-3,6-dioxo-5-(thiophen-2-yl)piperazin-2-yl)acetate (24)

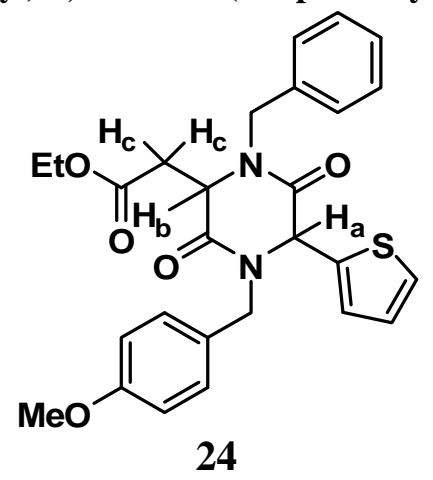

${ }^{1} \mathrm{H}$ NMR (500 MHz, $\mathrm{CDCl}_{3}$ ): $\delta$ 6.81-7.41 (m, 12, aryl), 5.47 (d, 1H, $\left.J=14.7 \mathrm{~Hz}, \mathrm{Bn}\right)$, $5.32(\mathrm{~d}, 1 \mathrm{H}, J=15.2 \mathrm{~Hz}, \mathrm{Bn}), 4.61\left(\mathrm{~m}, 1 \mathrm{H}, \mathrm{H}_{\mathrm{b}}\right), 4.04-4.15\left(\mathrm{~m}, 2 \mathrm{H},-\mathrm{OC}_{2} \mathrm{CH}_{3}\right), 3.96(\mathrm{~d}$, $1 \mathrm{H}, J=15.2 \mathrm{~Hz}, \mathrm{Bn}), 3.79$ (s, 3H), 3.59 (d, 1H, $J=14.7 \mathrm{~Hz}, \mathrm{Bn}), 3.28$ (dd, $1 \mathrm{H}, J=2.6$, $\left.17.2 \mathrm{~Hz}, \mathrm{H}_{\mathrm{c}}\right), 2.93\left(\mathrm{dd}, 1 \mathrm{H}, J=4.9,17.2 \mathrm{~Hz}, \mathrm{H}_{\mathrm{c}}\right), 1.17$ (t, $\left.3 \mathrm{H}, J=7.3 \mathrm{~Hz},-\mathrm{OCH}_{2} \mathrm{C}_{3}\right)$.

HRMS: EIMS $\left(\mathrm{M}^{+}\right)$calcd for $\mathrm{C}_{27} \mathrm{H}_{28} \mathrm{~N}_{2} \mathrm{O}_{5} \mathrm{~S} 492.1719$, found 492.1714.

\section{$\underline{{ }^{1} \text { H-NMR (Compound 24) }}$}

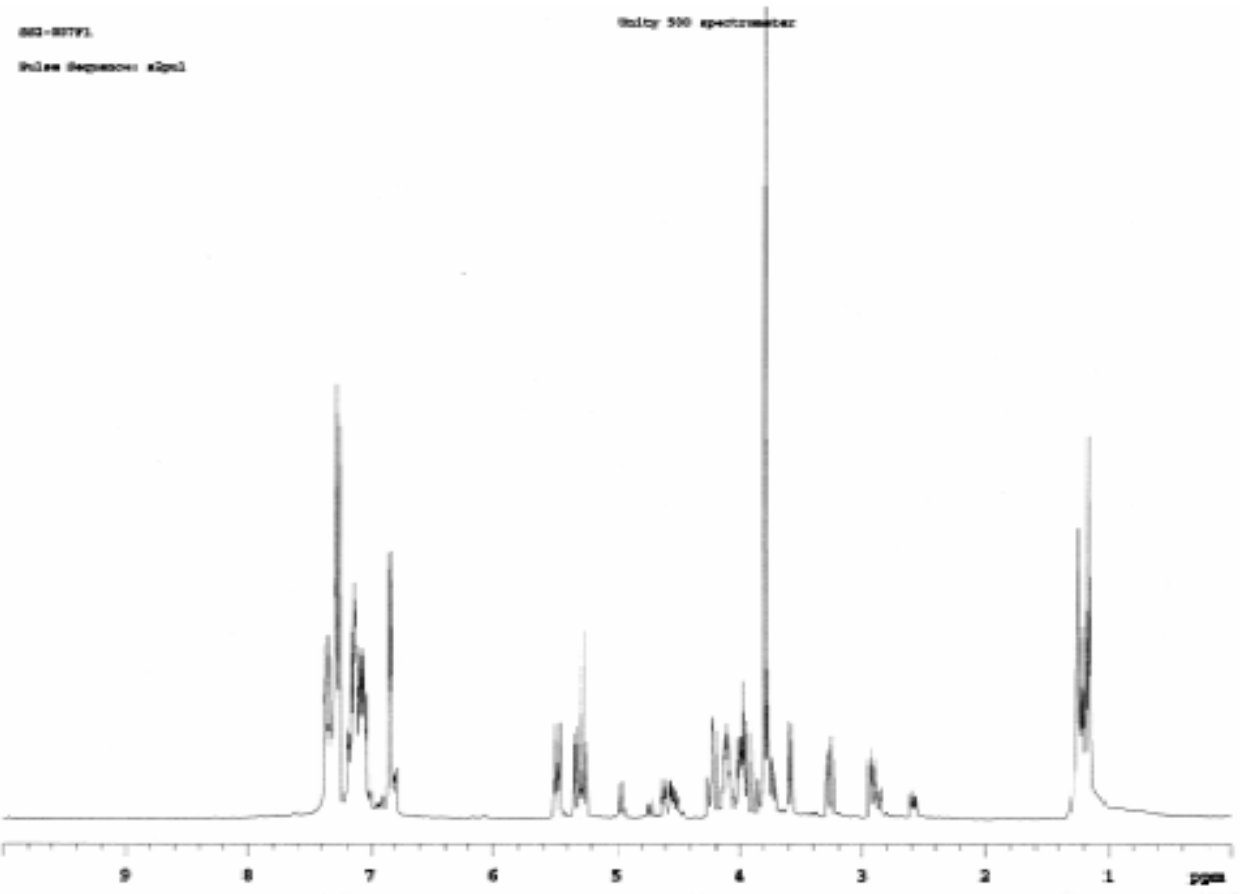


Substituted 2-azaspiro[4.5]deca-6,9-diene-3,8-diones (25) - X-Ray Structure below.

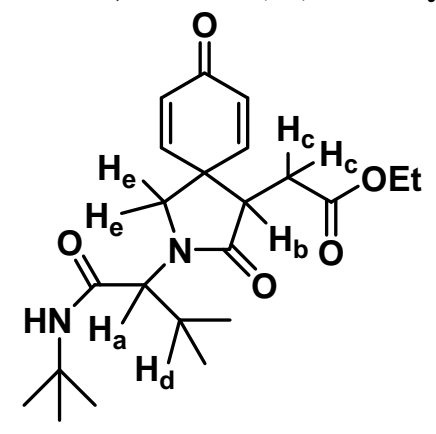

25

${ }^{1} \mathrm{H}$ NMR $\left(400 \mathrm{MHz}, \mathrm{CDCl}_{3}\right): \delta 6.62(\mathrm{~m}, 2 \mathrm{H}), 6.32(\mathrm{~m}, 2 \mathrm{H}), 5.79($ br s, $1 \mathrm{H}, \mathrm{NH}), 4.07(\mathrm{~m}$, $\left.2 \mathrm{H},-\mathrm{OC}_{2} \mathrm{CH}_{3}\right), 3.95\left(\mathrm{~d}, 1 \mathrm{H}, J=11 \mathrm{~Hz}, \mathrm{H}_{\mathrm{a}}\right), 3.78\left(\mathrm{~d}, 1 \mathrm{H}, J=10.4 \mathrm{~Hz}, \mathrm{H}_{\mathrm{e}}\right), 3.53(\mathrm{~d}, 1 \mathrm{H}$, $\left.J=10.4 \mathrm{~Hz}, \mathrm{H}_{\mathrm{e}}\right), 3.39\left(\mathrm{dd}, 1 \mathrm{H}, J=6.1,7.6 \mathrm{~Hz}, \mathrm{H}_{\mathrm{b}}\right), 2.65\left(\mathrm{dd}, 1 \mathrm{H}, J=6.1,16.6 \mathrm{~Hz}, \mathrm{H}_{\mathrm{c}}\right)$, $2.09\left(\mathrm{dd}, 1 \mathrm{H}, J=7.6,16.8 \mathrm{~Hz}, \mathrm{H}_{\mathrm{c}}\right), 1.20\left(\mathrm{t}, 3 \mathrm{H}, J=7.3 \mathrm{~Hz}, \mathrm{CH}_{3}\right), 0.95(\mathrm{~d}, 3 \mathrm{H}, J=6.1 \mathrm{~Hz}$, $\left.\mathrm{CHCH}_{3}\right), 0.90\left(\mathrm{~d}, 3 \mathrm{H}, J=4.9 \mathrm{~Hz}, \mathrm{CHCH}_{3}\right)$.

${ }^{13} \mathrm{C} \mathrm{NMR}\left(100 \mathrm{MHz}, \mathrm{CDCl}_{3}\right): \delta 184.9,172.6,171.3,168.2,149.2,145.9,131.4,130.9$, $62.8,61.1,50.8,50.1,48.1,47.4,30.3,28.6,27.3,19.3,19.3,14$.

HRMS: EIMS ( $\mathrm{M}^{+}$) calcd for $\mathrm{C}_{22} \mathrm{H}_{32} \mathrm{~N}_{2} \mathrm{O}_{5}$ 404.2311, found 404.2317.

$\underline{{ }^{1} \text { H-NMR (Compound 25) }}$ 


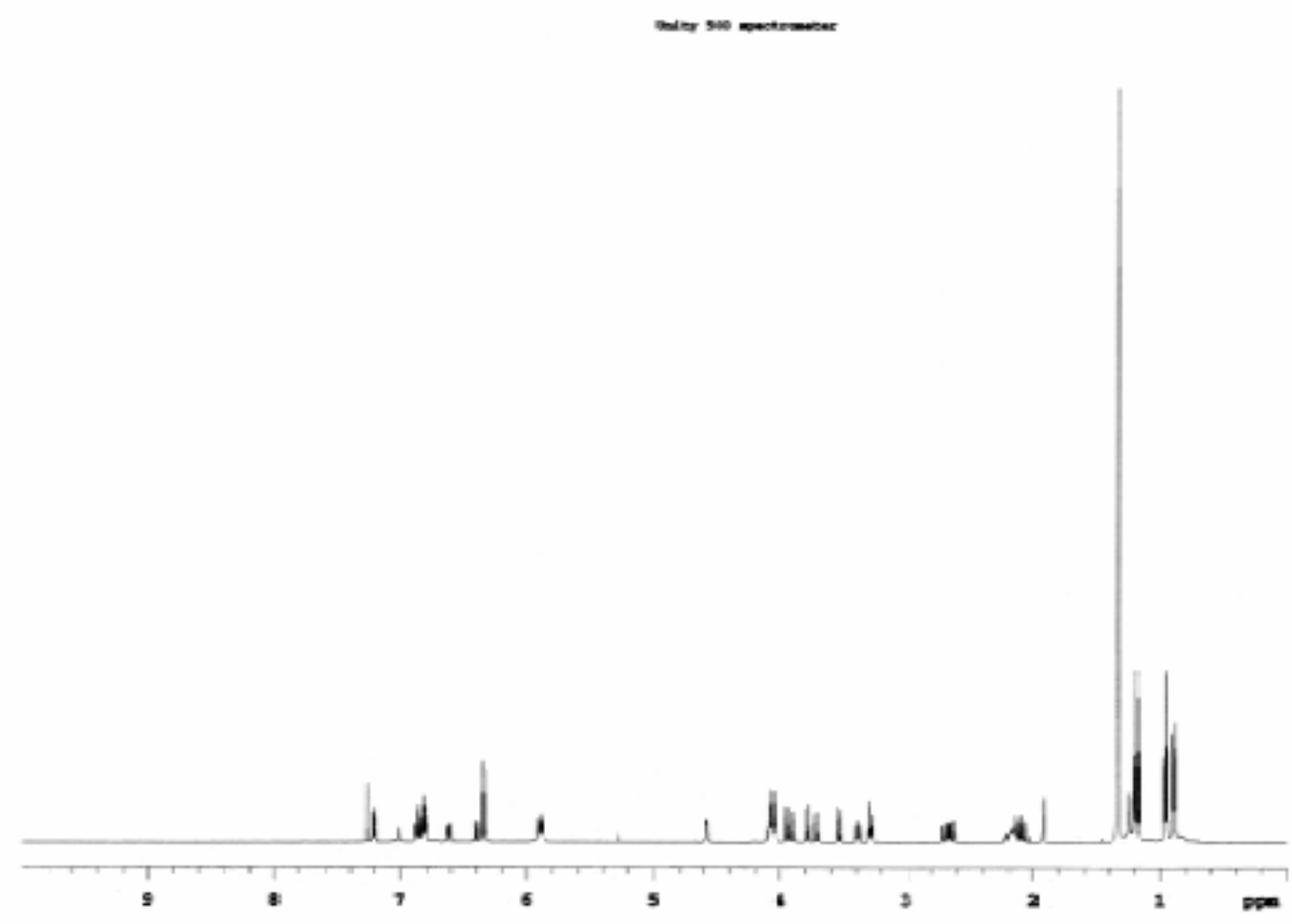

${ }^{13}$ C-NMR (Compound 25)

mati-solvo-1-5

riter cartise

nales sersencer saved

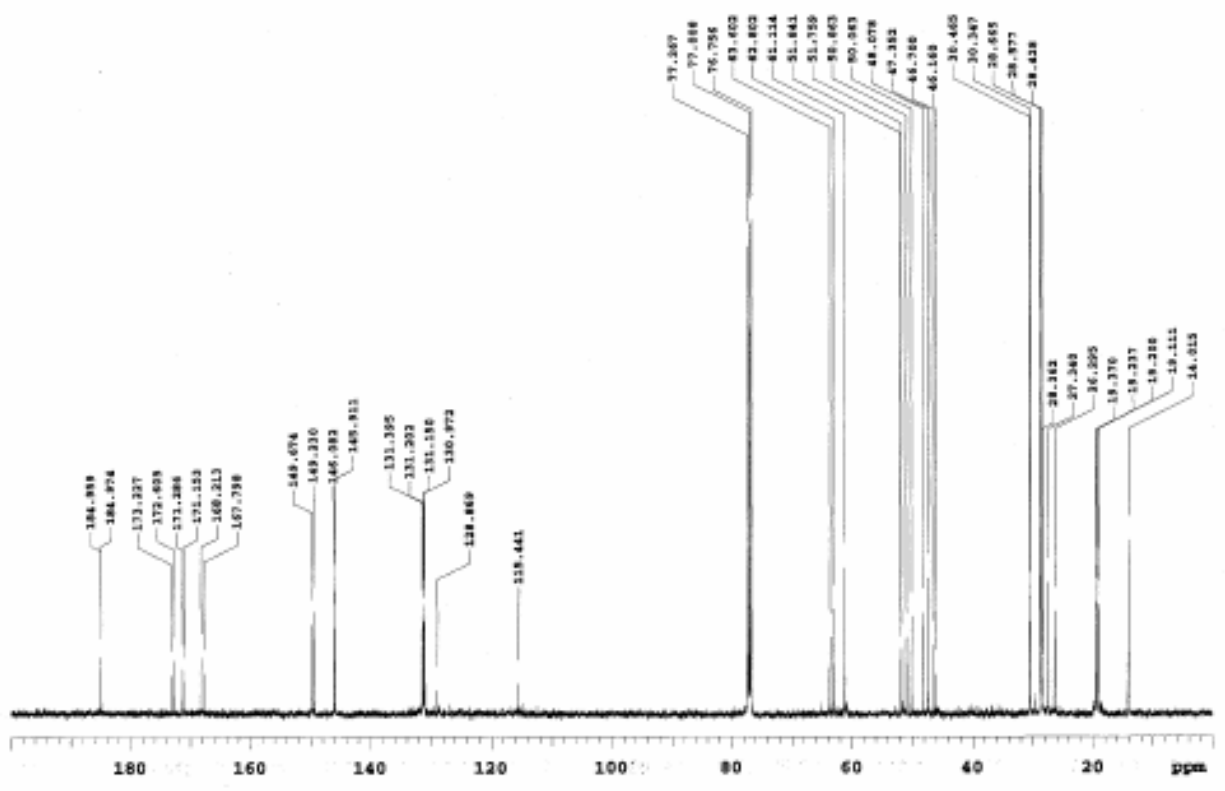


X-Ray Crystal Structure (Compound 25). There is severe disorder on one pendant arm (-COOEt) that makes the structure unpublishable in the Cambridge Crystallographic Data Centre. However, it does serve to identify the complex.

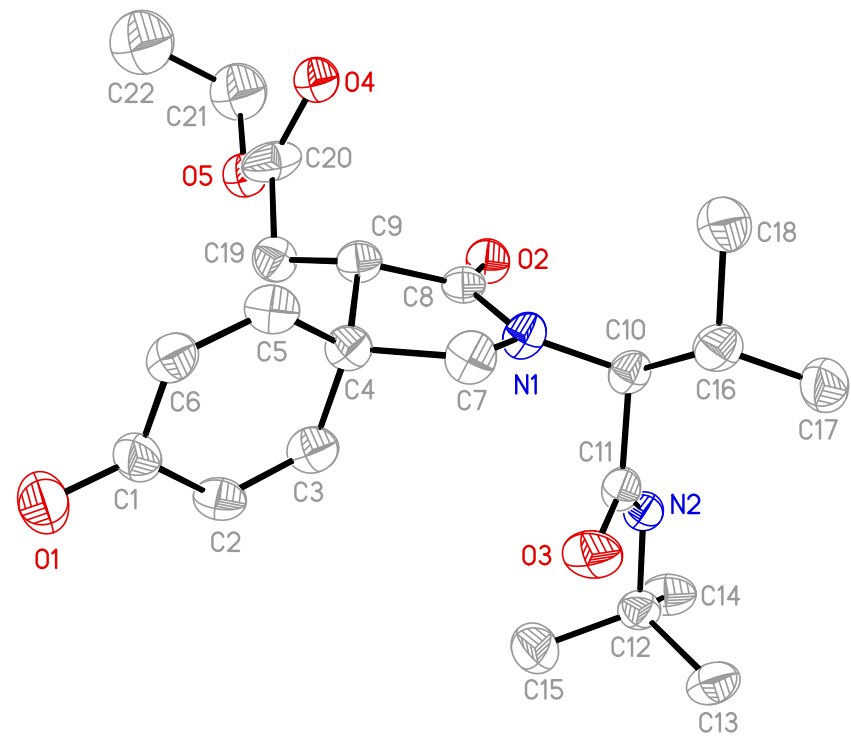

(E)-Ethyl 3-(N-(1-(tert-butylcarbamoyl)-2-methylpropyl)-N-(4-hydroxybenzyl)carbamoylacrylate (26)<smiles>CCOC(=O)/C=C/C(=O)N(Cc1ccc(O)cc1)C(C(=O)NC(C)(C)C)C(C)C</smiles>

26

${ }^{1} \mathrm{H}$ NMR (500 MHz, $\left.\mathrm{CDCl}_{3}\right): \delta 8.12(\mathrm{bs}, 1 \mathrm{H}), 7.3(\mathrm{~d}, 1 \mathrm{H}, J=15.2 \mathrm{~Hz}), 6.89(\mathrm{~d}, 2 \mathrm{H}, J=$ $8.6 \mathrm{~Hz}), 6.81(\mathrm{~d}, 1 \mathrm{H}, J=15.2 \mathrm{~Hz}), 6.6(\mathrm{~d}, 2 \mathrm{H}, J=8.6 \mathrm{~Hz}), 4.74(\mathrm{~d}, 1 \mathrm{H}, J=16.7 \mathrm{~Hz}), 4.5$ $(\mathrm{d}, 1 \mathrm{H}, J=16.7 \mathrm{~Hz}), 4.19$ (q, $\left.2 \mathrm{H}, J=7.1 \mathrm{~Hz},-\mathrm{OCH}_{2} \mathrm{CH}_{3}\right), 2.5$ (bs, $\left.1 \mathrm{H}\right), 1.26$ (t, 3H, $J=$ $7.1 \mathrm{~Hz}), 0.94(\mathrm{~d}, 3 \mathrm{H}, J=6.6 \mathrm{~Hz}), 0.81(\mathrm{~d}, 3 \mathrm{H}, J=6.6 \mathrm{~Hz})$.

${ }^{13} \mathrm{C}$ NMR $\left(125 \mathrm{MHz}, \mathrm{CDCl}_{3}\right): \delta 169.5,166.9,165.4,156.3,134.1,132.1,127.8,127.1$, $115.7,61.2,51.4,28.7,28.5,27.3,19.5,18.9,14.0$.

HRMS: EIMS $\left(\mathrm{M}^{+}\right)$calcd for $\mathrm{C}_{22} \mathrm{H}_{32} \mathrm{~N}_{2} \mathrm{O}_{5}$ 404.2311, found 404.2321.

\section{$\underline{{ }^{1} \text { H-NMR (Compound 26) }}$}



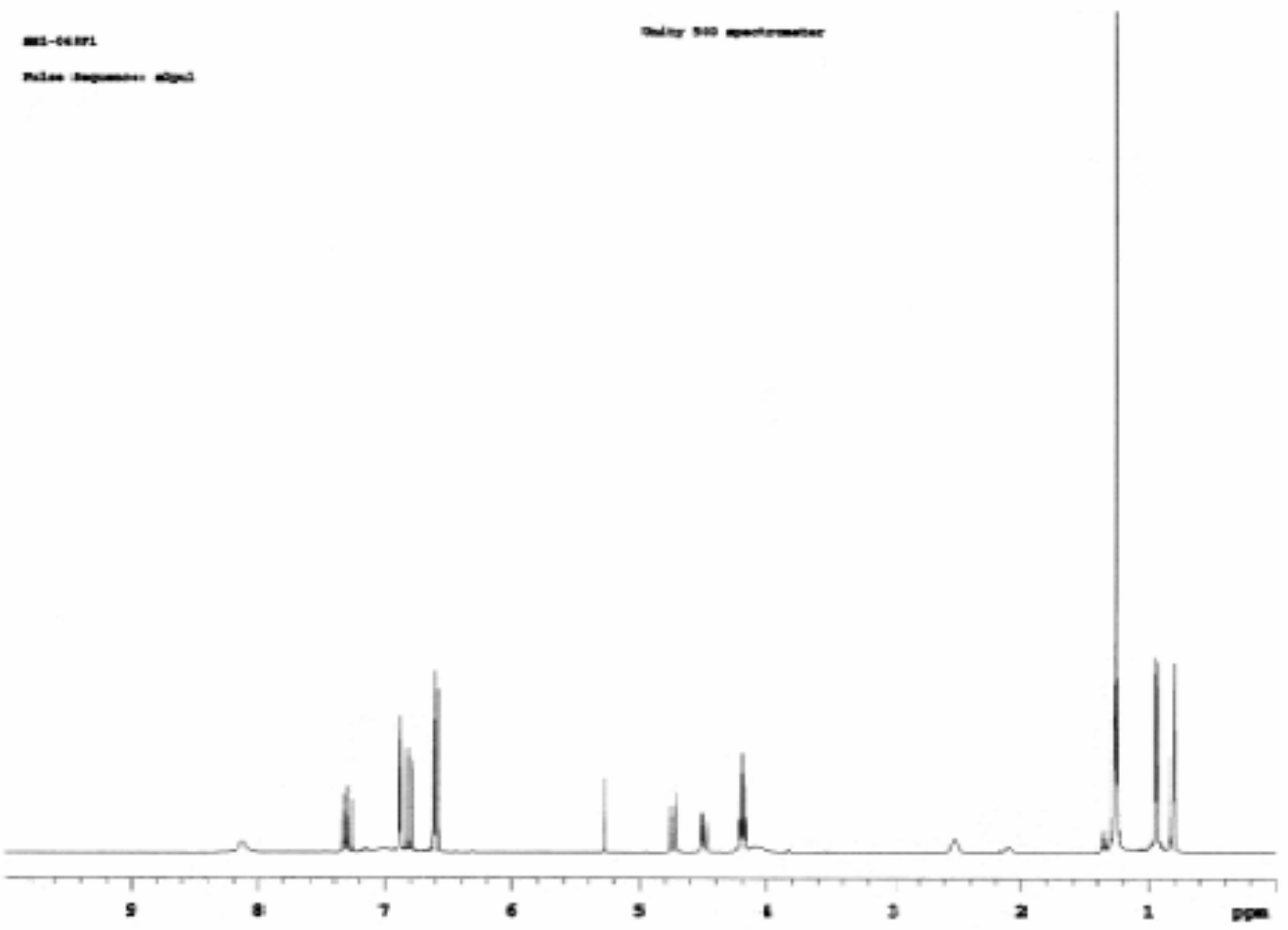

${ }^{13}$ C-NMR (Compound 26)

$$
\text { nil-6esn }
$$

nitor sps meetroneter

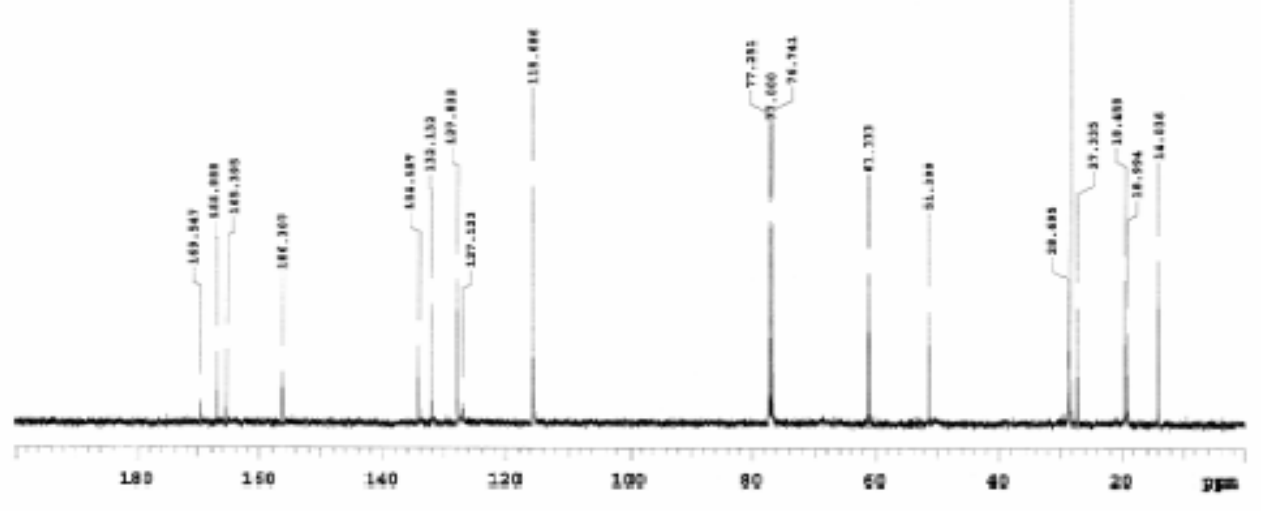




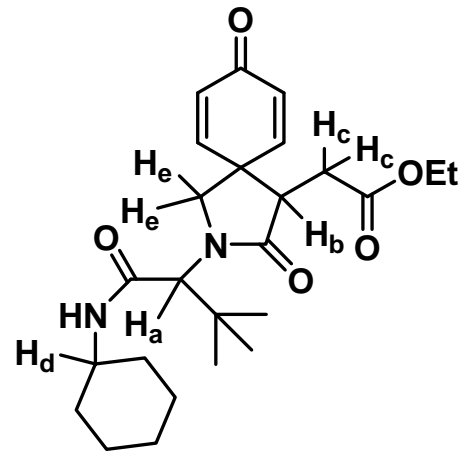

27

${ }^{1} \mathrm{H}$ NMR (500 MHz, $\left.\mathrm{CDCl}_{3}\right): \delta$ 6.81-6.93 (m, 2H), 6.33-6.47 (m, 2H), $5.62(\mathrm{~d}, 1 \mathrm{H}, J=$ $7.6 \mathrm{~Hz},-\mathrm{N} \underline{\mathrm{H}}$ ), $4.25\left(\mathrm{~s}, 1 \mathrm{H}, \mathrm{H}_{\mathrm{a}}\right), 4.08\left(\mathrm{dq}, 2 \mathrm{H}, J=1.4,7.2 \mathrm{~Hz},-\mathrm{OCH}_{2} \mathrm{CH}_{3}\right), 3.69-3.78(\mathrm{~m}$, $3 \mathrm{H}, \mathrm{H}_{\mathrm{e}}$ and $\mathrm{H}_{\mathrm{d}}$ overlap), $3.29\left(\mathrm{dd}, 1 \mathrm{H}, J=6.1,7.6 \mathrm{~Hz}, \mathrm{H}_{\mathrm{b}}\right), 2.90(\mathrm{dd}, 1 \mathrm{H}, J=5.9,16.6 \mathrm{~Hz}$, $\mathrm{H}_{\mathrm{c}}$ ), $2.13\left(\mathrm{dd}, 1 \mathrm{H}, J=7.6,16.8 \mathrm{~Hz}, \mathrm{H}_{\mathrm{c}}\right), 1.83-1.97$ (m, 3H, Cy), 1.66-1.77 (m, 3H, Cy), 1.57-1.65 (m, 2H, Cy), 1.30-1.41 (m, 2H, Cy), $1.20\left(\mathrm{t}, 3 \mathrm{H}, J=7.3 \mathrm{~Hz},-\mathrm{OCH}_{2} \mathrm{CH}_{3}\right)$.

${ }^{13} \mathrm{C}$ NMR $\left(125 \mathrm{MHz}, \mathrm{CDCl}_{3}\right): \delta 185.1,173.8,171.3,166.6,149.7,146.1,131.4,131.3$, 64.1, 61.1, 53.9, 48.5, 47.5, 46.6, 36.1, 33.1, 32.9, 30.7, 27.6, 25.4, 24.8, 24.7, 14.1.

HRMS: EIMS ( $\left.\mathrm{M}^{+}\right)$calcd for $\mathrm{C}_{25} \mathrm{H}_{36} \mathrm{~N}_{2} \mathrm{O}_{5} 444.2624$, found 444.2632

${ }^{1}$ H-NMR (Compound 27)

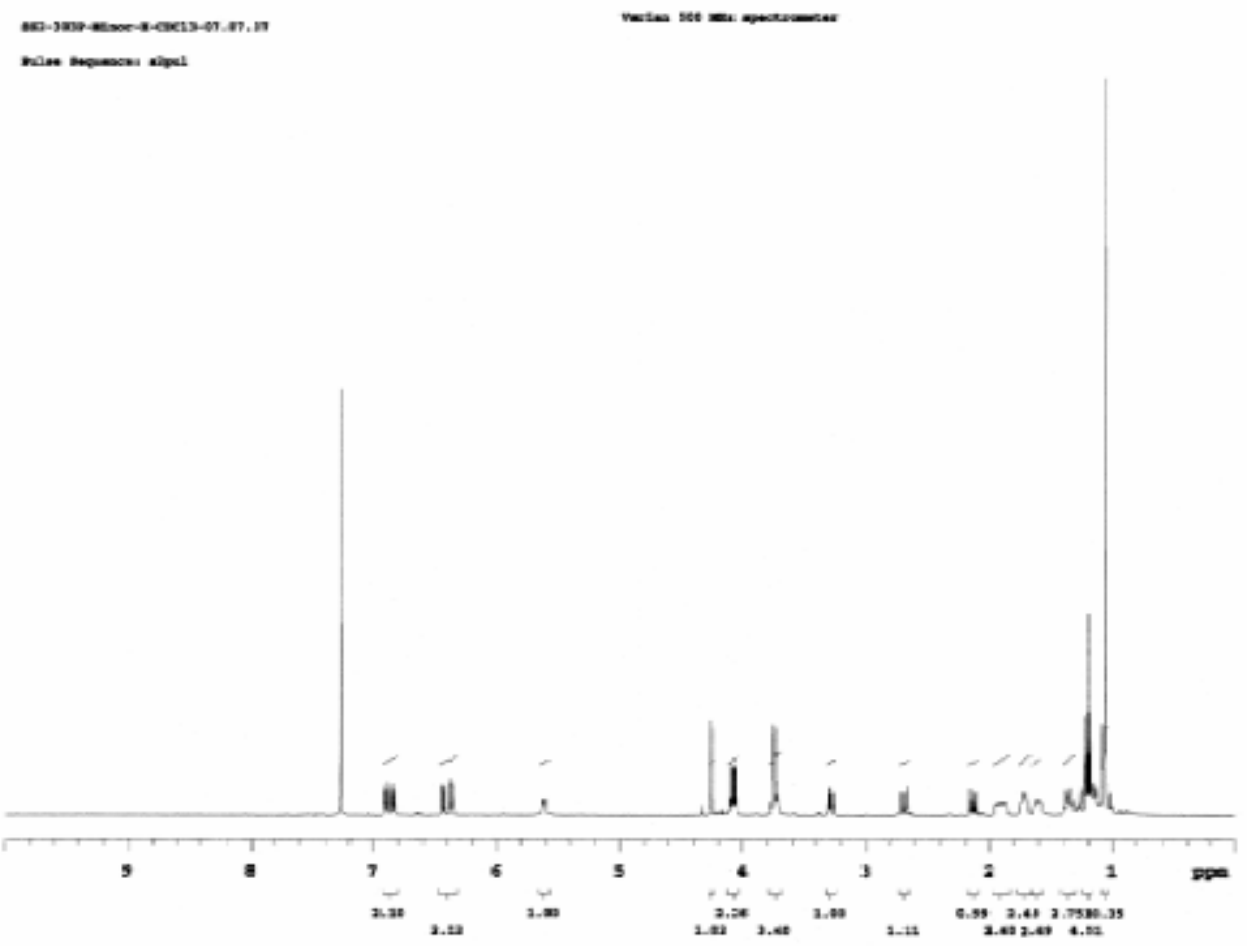

${ }^{13}$ C-NMR (Compound 27) 


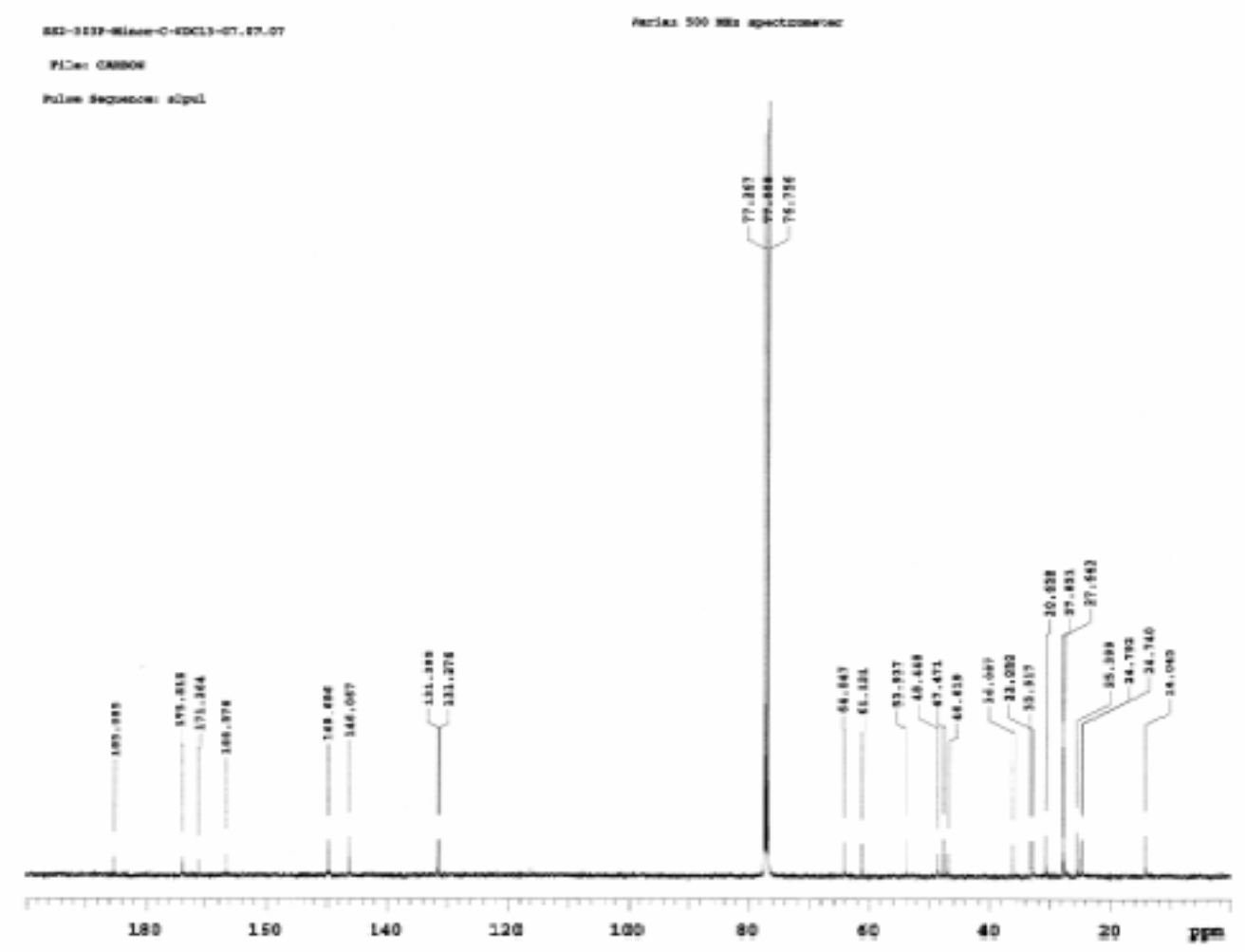

(E)-ethyl 4-((1-(benzylamino)-3,3-dimethyl-1-oxobutan-2-yl)(cyclohexylmethyl)amino)-4-oxobut-2enoate (28)<smiles>CCOC(=O)/C=C/C(=O)N(CC1CCCCC1)C(C)(C(=O)NCc1ccc(O)cc1)C(C)(C)C</smiles>

28

${ }^{1} \mathrm{H}$ NMR $\left(400 \mathrm{MHz}, \mathrm{CDCl}_{3}\right): \delta 7.35(\mathrm{~d}, 1 \mathrm{H}, J=15.4 \mathrm{~Hz},-\mathrm{CH}=\mathrm{CH}-), 7.19-7.31(\mathrm{~m}, 5 \mathrm{H}$, aryl), $6.69(\mathrm{~d}, 1 \mathrm{H}, J=15.4 \mathrm{~Hz},-\mathrm{CH}=\mathrm{C} \underline{\mathrm{H}}-), 4.47(\mathrm{dd}, 1 \mathrm{H}, J=6.5,14.6 \mathrm{~Hz}$, benzyl), 4.17$4.32\left(\mathrm{~m}, 4 \mathrm{H},-\mathrm{OC} \underline{\mathrm{H}}_{2} \mathrm{CH}_{3}\right.$, benzyl and $\mathrm{H}_{\mathrm{a}}$ overlap), 3.72 (bs, $1 \mathrm{H}$, cyclohexylmethyl), 3.25 (bs, $1 \mathrm{H}$, cyclohexylmethyl), 1.40-1.72 (m, 8H, cyclohexyl), 1.29 (t, $3 \mathrm{H}, J=7.3 \mathrm{~Hz}$, $\mathrm{OCH}_{2} \mathrm{C}_{3}$ ), 1.06 (s, 9H, t-butyl), 0.70-0.94 (m, 3H, cyclohexyl).

${ }^{13} \mathrm{C}$ NMR $\left(100 \mathrm{MHz}, \mathrm{CDCl}_{3}\right): \delta 169.5,166.7,165.5,137.9,134.6,131.1,128.5,127.8$, $127.2,61.1,43.4,36.8,30.8,29.6,28.1,26.1,25.8,25.7,14.1$.

MS: EIMS $(\mathrm{M}+\mathrm{H})$ calcd for $\mathrm{C}_{26} \mathrm{H}_{38} \mathrm{~N}_{2} \mathrm{O}_{4} 443.2832$, found 443.3

\section{$\underline{{ }^{1} \text { H-NMR (Compound 28) }}$}




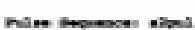

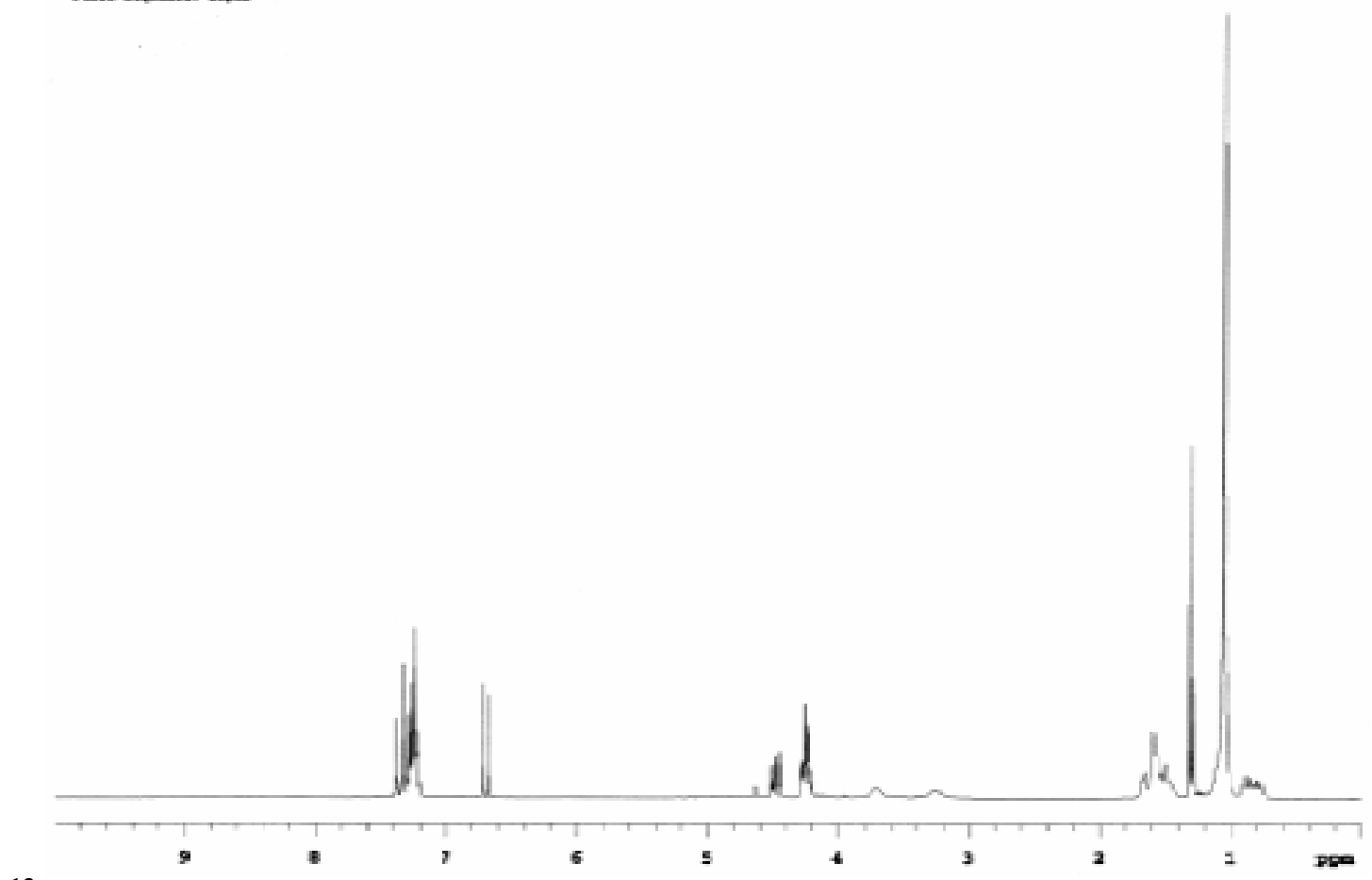

${ }^{13}$ C-NMR (Compound 28)

cantise spectors

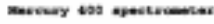

naine serseneren sapes

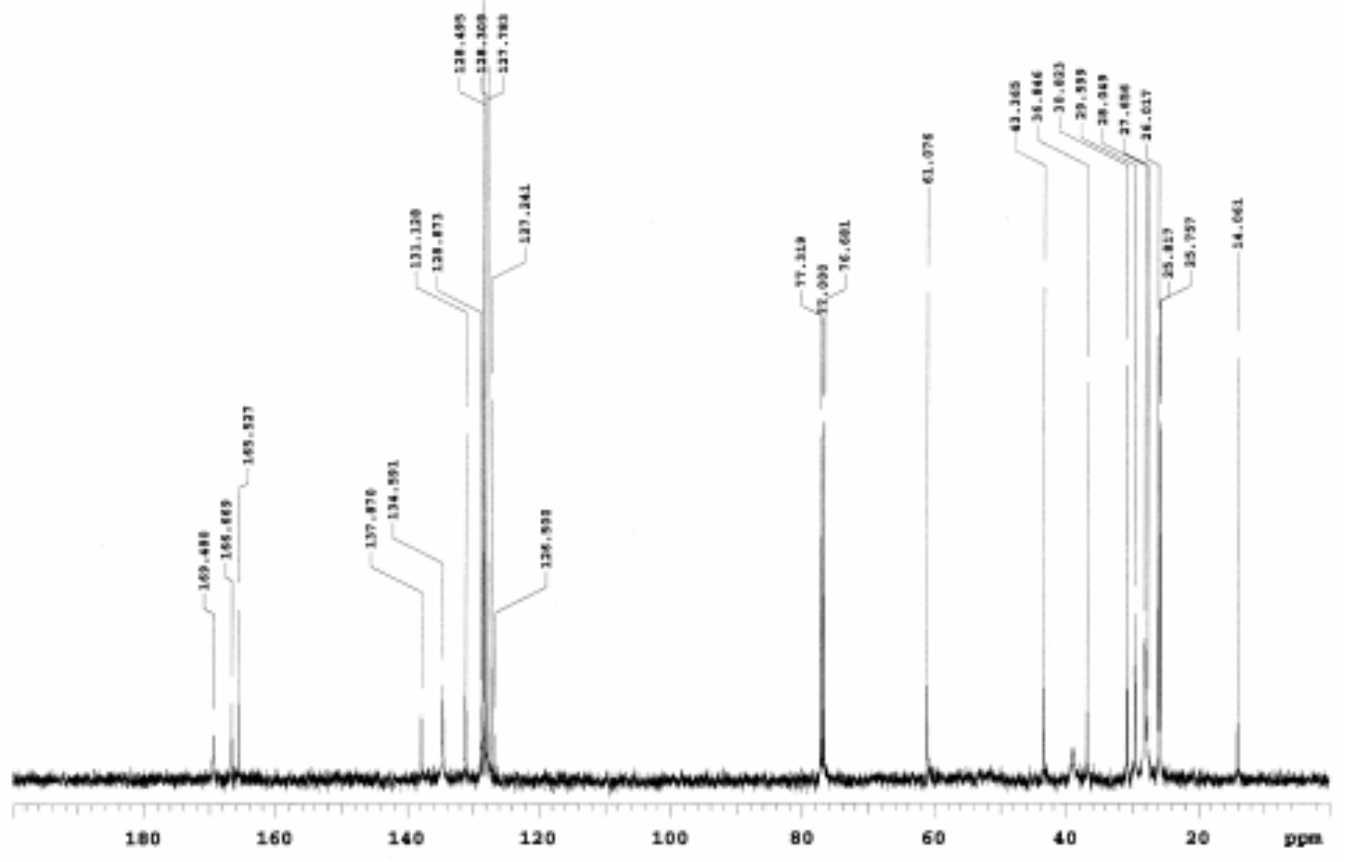


Substituted 2-azaspiro[4.5]deca-6,9-diene-3,8-diones (29)

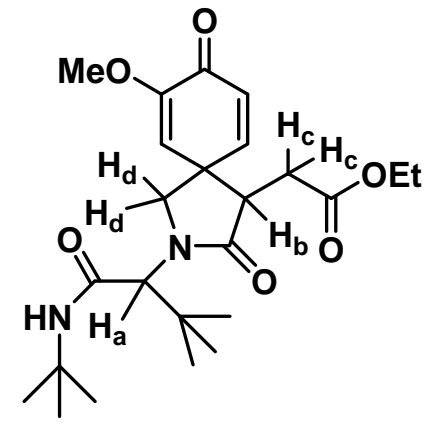

29

${ }^{1} \mathrm{H}$ NMR (500 MHz, $\left.\mathrm{CDCl}_{3}\right): \delta 6.91(\mathrm{dd}, 1 \mathrm{H}, J=2.7,10.1 \mathrm{~Hz}), 6.38(\mathrm{~d}, 1 \mathrm{H}, J=10.1 \mathrm{~Hz})$, $5.57(\mathrm{~d}, 1 \mathrm{H}, J=2.7 \mathrm{~Hz}), 5.51$ (br s, $1 \mathrm{H}, \mathrm{NH}), 4.31$ (d, 1H, J=10.1 Hz, $\left.\mathrm{H}_{\mathrm{d}}\right), 4.28(\mathrm{~s}, 1 \mathrm{H}$, $\left.\mathrm{H}_{\mathrm{a}}\right), 4.08\left(\mathrm{~m}, 2 \mathrm{H},-\mathrm{OCH}_{2} \mathrm{CH}_{3}\right), 3.78\left(\mathrm{~d}, 1 \mathrm{H}, J=10.1 \mathrm{~Hz}, \mathrm{H}_{\mathrm{d}}\right), 3.61\left(\mathrm{~s}, 3 \mathrm{H}, \mathrm{OCH}_{3}\right), 3.39$ $\left(\mathrm{dd}, 1 \mathrm{H}, J=5.8,7.4 \mathrm{~Hz}, \mathrm{H}_{\mathrm{b}}\right), 2.66\left(\mathrm{dd}, 1 \mathrm{H}, J=6.1,16.6 \mathrm{~Hz}, \mathrm{H}_{\mathrm{c}}\right), 2.09$ (dd, $1 \mathrm{H}, J=7.3$, $16.8 \mathrm{~Hz}, \mathrm{H}_{\mathrm{c}}$ ), 1.34 (s, 9H, t-butyl), 1.21 (t, 3H, $J=7.3 \mathrm{~Hz}, \mathrm{CH}_{3}$ ), 1.08 (s, 9H, t-butyl).

${ }^{13} \mathrm{C}$ NMR $\left(125 \mathrm{MHz}, \mathrm{CDCl}_{3}\right): \delta 180.4,173.9,171.6,167.1,152.7,146.4,130.7,116.5$, $64.2,61.2,55.1,54.8,51.9,48.3,47.3,36.2,30.4,28.6,27.6,14.0$.

HRMS: EIMS $\left(\mathrm{M}^{+}\right)$calcd for $\mathrm{C}_{24} \mathrm{H}_{36} \mathrm{~N}_{2} \mathrm{O}_{6} 448.2573$, found 448.2582.

\section{$\underline{{ }^{1} \text { H-NMR (Compound 29) } \text { Mixture of Diastereomers }}$}
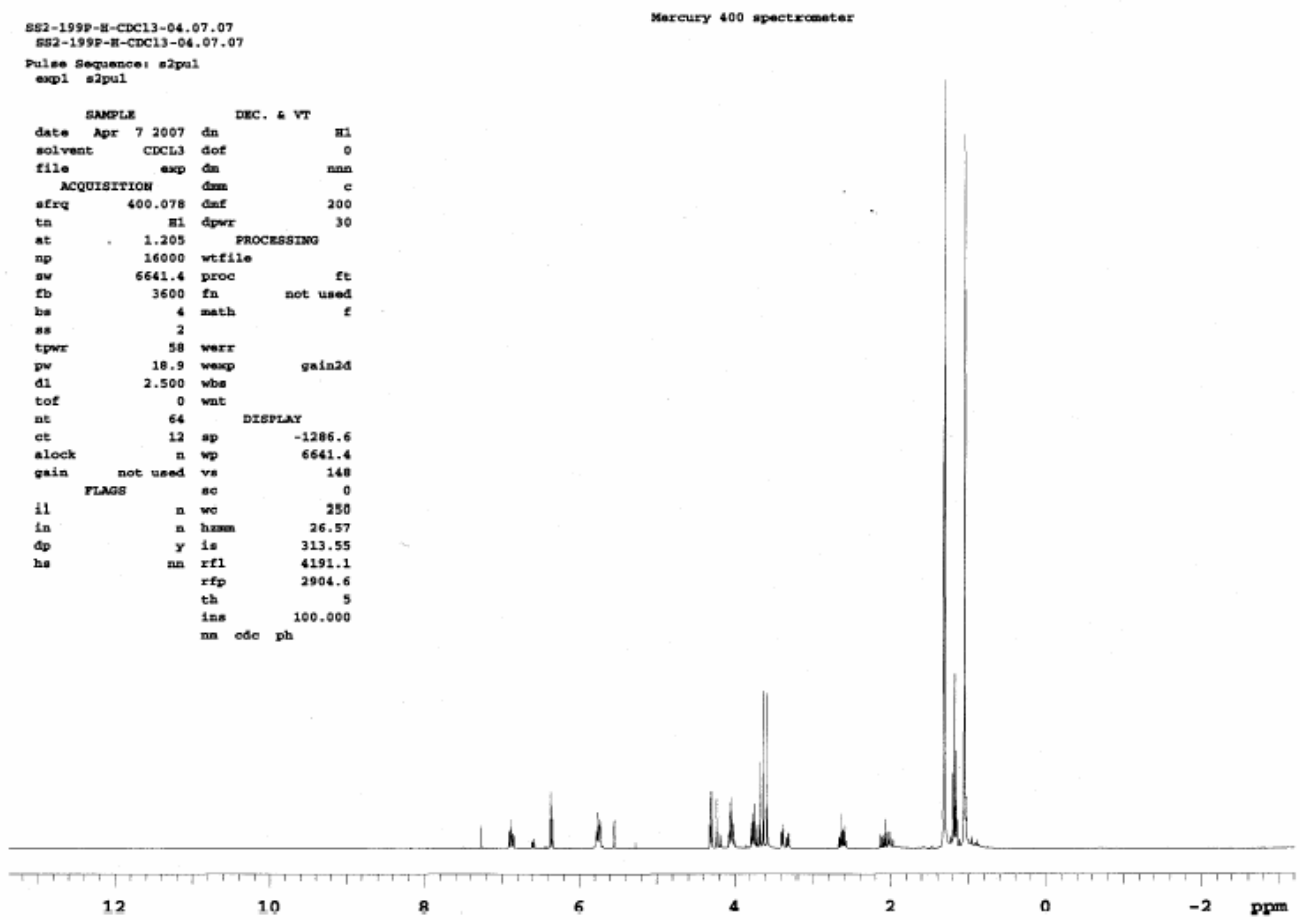


\section{${ }^{13}$ C-NMR (Compound 29) Mixture of Diastereomers}

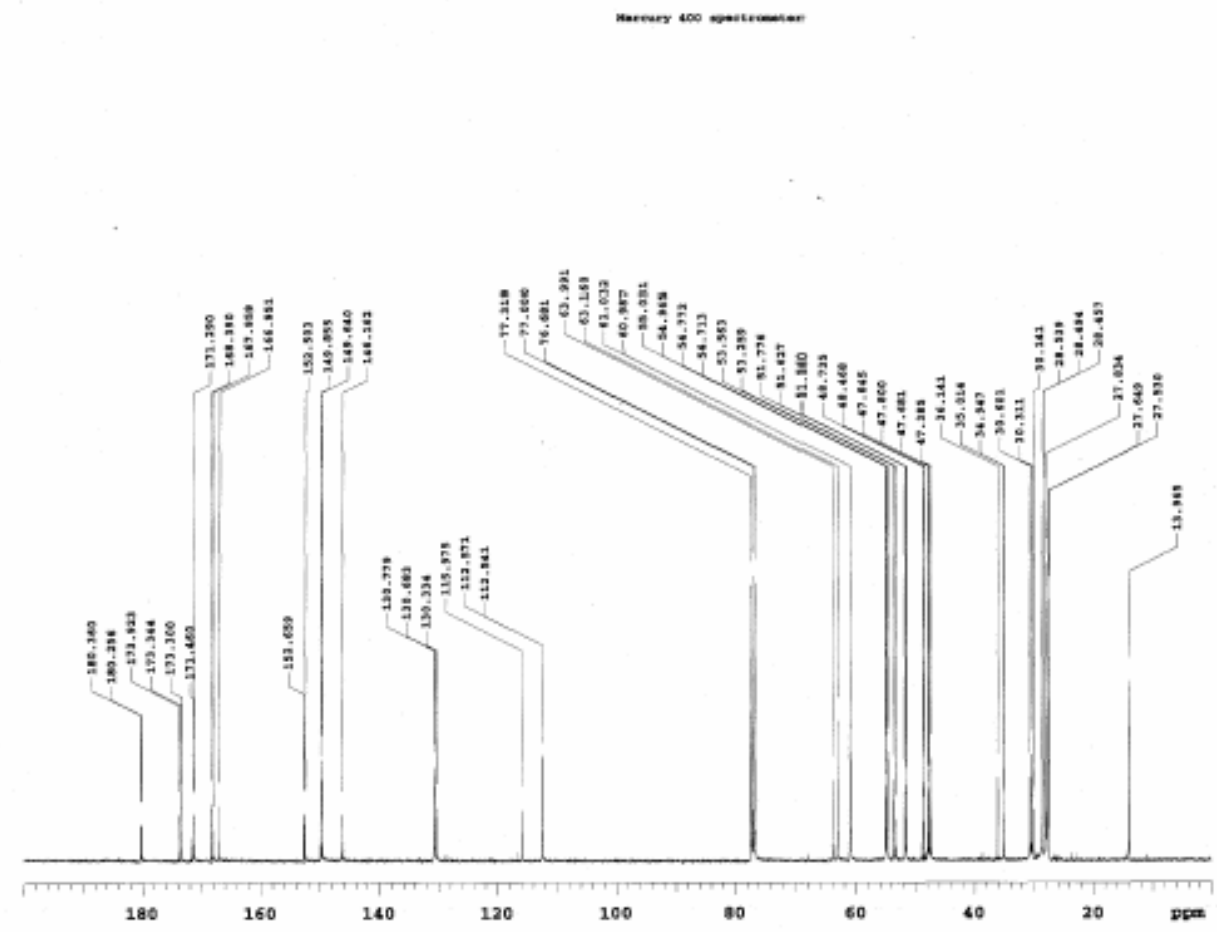

(E)-Ethyl 3-(N-(1-(tert-butylcarbamoyl)-2,2-dimethylpropyl)-N-(4-hydroxy-3methoxybenzyl)carbamoylacrylate (30)<smiles>CCOC(=O)/C=C/C(=O)N(Cc1ccc(O)c(OC)c1)C(C(=O)NC(C)(C)C)C(C)(C)C</smiles>

30

${ }^{1} \mathrm{H}$ NMR $\left(400 \mathrm{MHz}, \mathrm{CDCl}_{3}\right): \delta 7.16(\mathrm{~d}, 1 \mathrm{H}, J=14.6 \mathrm{~Hz}), 6.4-6.74(\mathrm{~m}, 4 \mathrm{H}), 5.38(\mathrm{~d}, 1 \mathrm{H}$, $J=17 \mathrm{~Hz}), 4.98(\mathrm{bs}, 1 \mathrm{H}), 4.65(\mathrm{~d}, 1 \mathrm{H}, J=17 \mathrm{~Hz}), 4.06\left(\mathrm{q}, 2 \mathrm{H}, J=7.3 \mathrm{~Hz},-\mathrm{OC}_{2} \mathrm{CH}_{3}\right)$, $3.73(\mathrm{~s}, 3 \mathrm{H}), 1.18(\mathrm{t}, 3 \mathrm{H}, J=7.3 \mathrm{~Hz})$.

${ }^{13} \mathrm{C}$ NMR $\left(100 \mathrm{MHz}, \mathrm{CDCl}_{3}\right): \delta 167.9,167.2,165.3,146.8,144.6,134.1,134.0,131.3$, $118.4,114.5,108.8,60.9,60.2,55.7,51.2,36.9,28.1,27.4,13.8$.

HRMS: EIMS $\left(\mathrm{M}^{+}\right)$calcd for $\mathrm{C}_{24} \mathrm{H}_{36} \mathrm{~N}_{2} \mathrm{O}_{6} 448.2573$, found 448.2578

\section{$\underline{{ }^{1} \text { H-NMR (Compound 30) }}$}




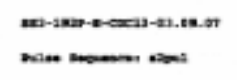

nom an wher

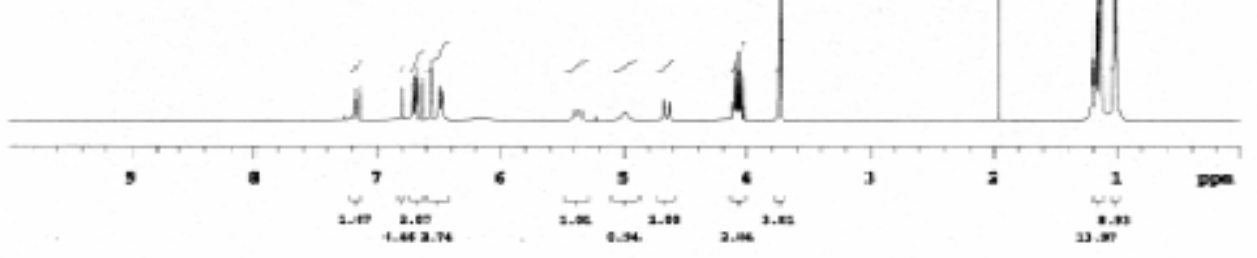

${ }^{13}$ C-NMR (Compound 30)

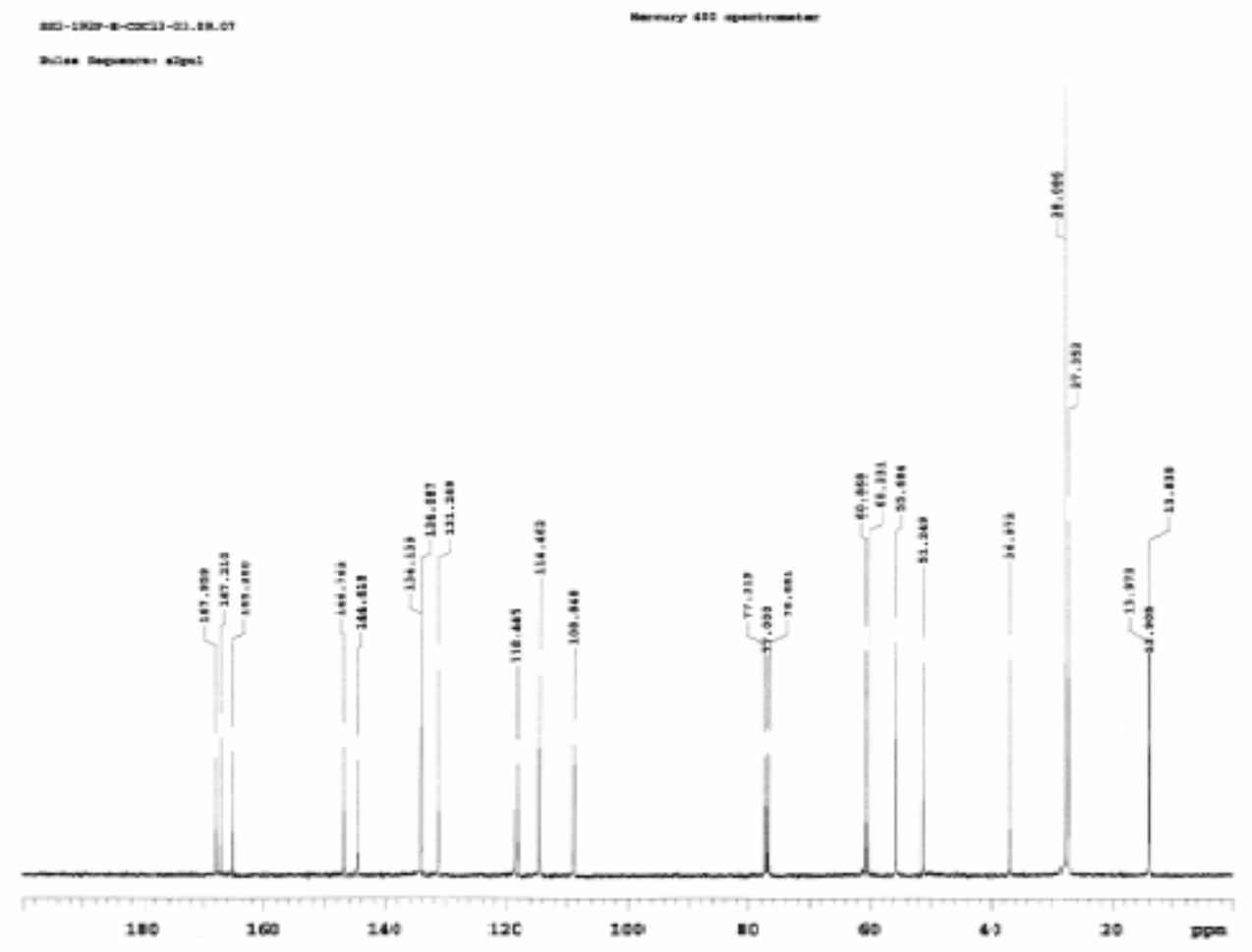

S43 
Substituted 2-azaspiro[4.5]deca-6,9-diene-3,8-diones (31)

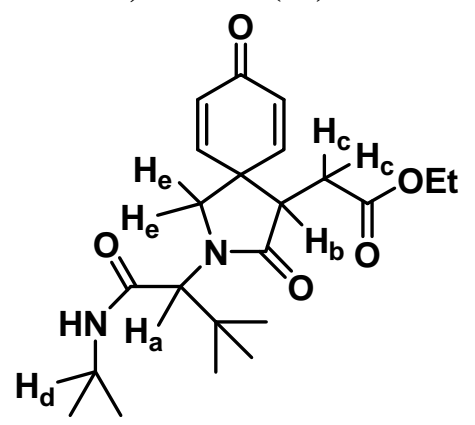

31

${ }^{1} \mathrm{H}$ NMR $\left(500 \mathrm{MHz}, \mathrm{CDCl}_{3}\right): \delta 6.89(\mathrm{~m}, 1 \mathrm{H}), 6.63(\mathrm{~m}, 1 \mathrm{H}), 6.36(\mathrm{~m}, 2 \mathrm{H}), 5.65(\mathrm{~d}, 1 \mathrm{H}, J$ $=7.3 \mathrm{~Hz},-\mathrm{N} \underline{\mathrm{H}}), 4.33\left(\mathrm{~s}, 1 \mathrm{H}, \mathrm{H}_{\mathrm{a}}\right), 4.17\left(\mathrm{~d}, 1 \mathrm{H}, J=10.4 \mathrm{~Hz}, \mathrm{H}_{\mathrm{e}}\right), 4.00-4.12\left(\mathrm{~m}, 3 \mathrm{H}, \mathrm{H}_{\mathrm{d}}\right.$ and - OC트2CH3 overlap), $3.74\left(\mathrm{~d}, 1 \mathrm{H}, J=10.4 \mathrm{~Hz}, \mathrm{H}_{\mathrm{e}}\right), 3.38\left(\mathrm{dd}, 1 \mathrm{H} . J=6.4,7.6 \mathrm{~Hz}, \mathrm{H}_{\mathrm{b}}\right.$ ), $2.64\left(\mathrm{dd}, 1 \mathrm{H}, J=5.8,16.7 \mathrm{~Hz}, \mathrm{H}_{\mathrm{c}}\right), 2.07\left(\mathrm{dd}, 1 \mathrm{H}, J=7.6,16.7 \mathrm{~Hz}, \mathrm{H}_{\mathrm{e}}\right), 1.20(\mathrm{t}, 3 \mathrm{H}, J=$ $\left.7.3 \mathrm{~Hz},-\mathrm{OCH}_{2} \mathrm{CH}_{3}\right), 1.16$ (d, $\left.3 \mathrm{H}, J=6.1 \mathrm{~Hz}, i-\mathrm{Pr}\right), 1.15$ (d, 3H, $\left.J=6.1 \mathrm{~Hz}, i-\operatorname{Pr}\right), 1.07$ (s, $9 \mathrm{H}, t-\mathrm{Bu})$.

${ }^{13} \mathrm{C}$ NMR $\left(125 \mathrm{MHz}, \mathrm{CDCl}_{3}\right): \delta 185.1,173.3,171.4,167.7,149.3,145.9,131.5,131.0$, $63.2,61.1,52.6,47.6,47.0,41.5,35.1,30.4,27.8,22.6,14.0$.

HRMS: EIMS ( $\mathrm{M}^{+}$) calcd for $\mathrm{C}_{22} \mathrm{H}_{32} \mathrm{~N}_{2} \mathrm{O}_{5}$ 404.2311, found 404.2323.

${ }^{1}$ H-NMR (Compound 31) 


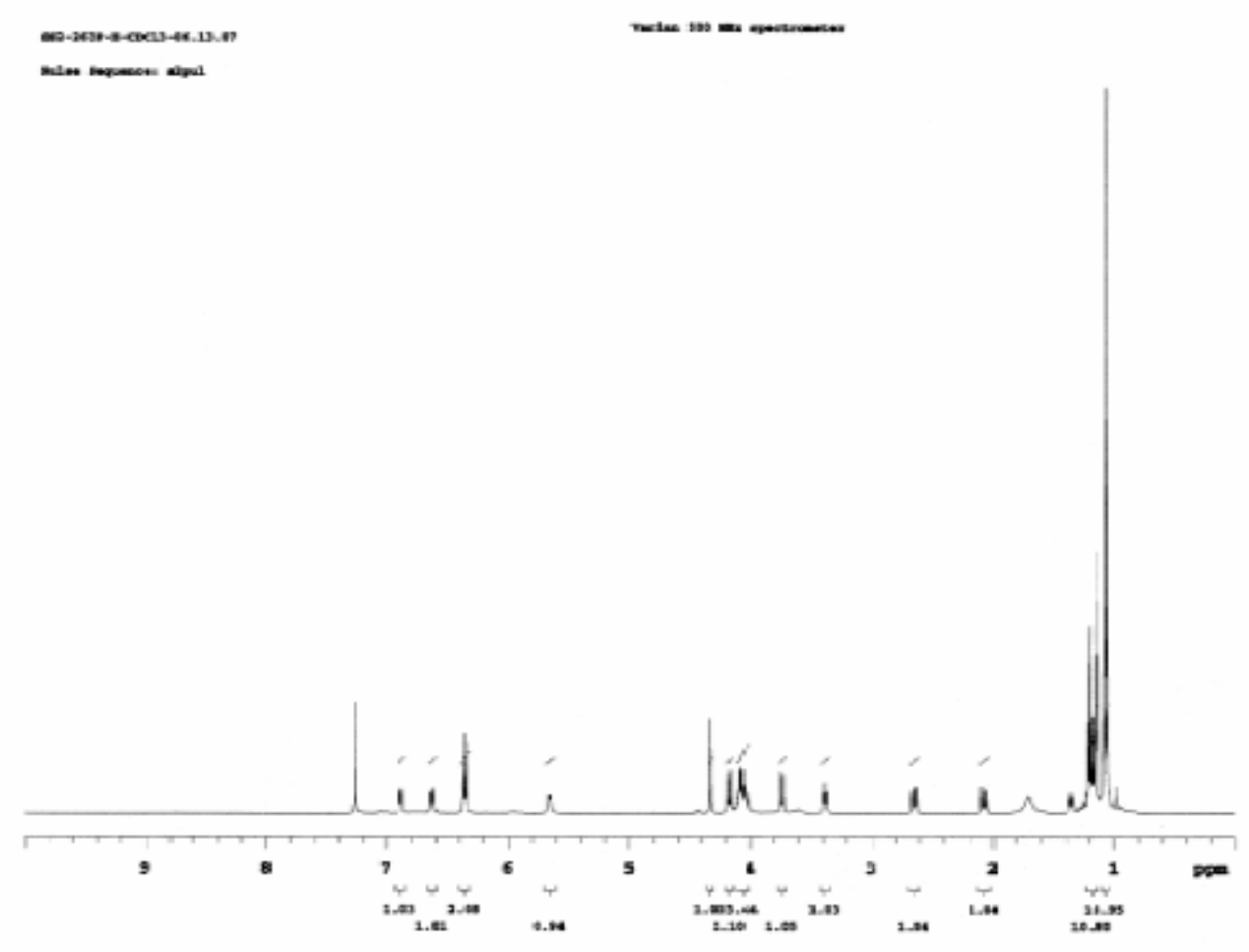

${ }^{13}$ C-NMR (Compound 31)
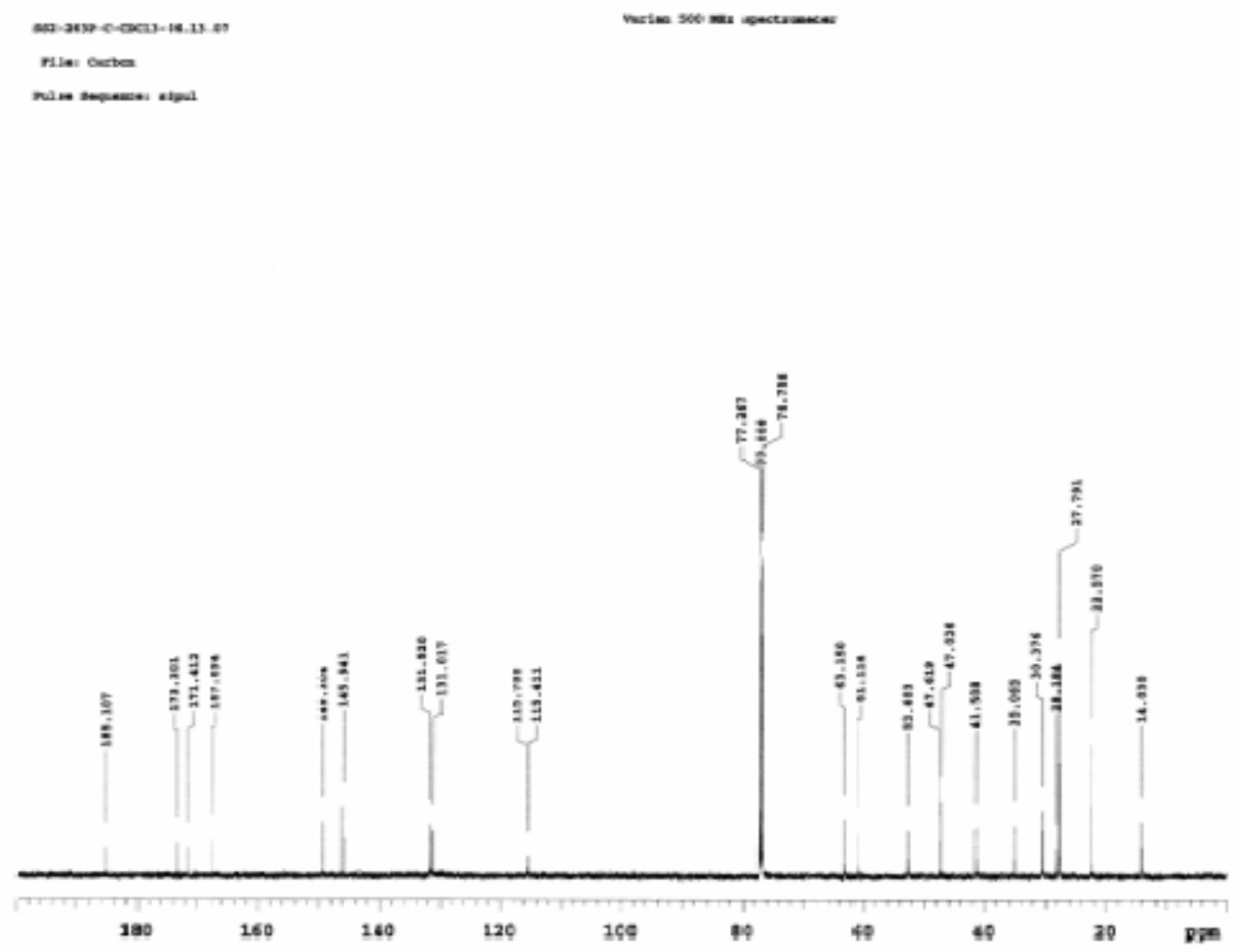
(E)-ethyl 4-((4-hydroxybenzyl)(1-(isopropylamino)-3,3-dimethyl-1-oxobutan-2-yl)amino)-4-oxobut-2enoate (32)

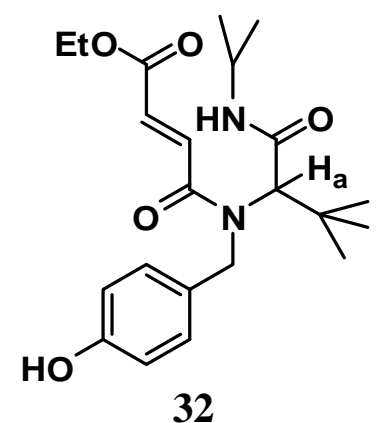

${ }^{1} \mathrm{H}$ NMR $\left(300 \mathrm{MHz}, \mathrm{CDCl}_{3}\right): \delta 7.19(\mathrm{~d}, 1 \mathrm{H}, J=15.3 \mathrm{~Hz},-\mathrm{CH}=\mathrm{CH}-), 6.83(\mathrm{~d}, 2 \mathrm{H}, J=8.5$ $\mathrm{Hz}$, aryl), $6.76(\mathrm{~d}, 1 \mathrm{H}, J=15.3 \mathrm{~Hz},-\mathrm{CH}=\mathrm{CH}-), 6.55(\mathrm{~d}, 2 \mathrm{H}, J=8.5 \mathrm{~Hz}, \operatorname{aryl}), 5.36$ (d, $1 \mathrm{H}, J=17.1 \mathrm{~Hz}, \mathrm{Bn}), 4.96\left(\mathrm{~s}, 1 \mathrm{H}, \mathrm{H}_{\mathrm{a}}\right), 4.66(\mathrm{~d}, 1 \mathrm{H}, J=17.1 \mathrm{~Hz}), 4.17(\mathrm{dq}, 2 \mathrm{H}, J=2.4$, $\left.7.3 \mathrm{~Hz},-\mathrm{OC}_{2} \mathrm{CH}_{3}\right), 3.84$ (sept, $\left.1 \mathrm{H}, J=6.7 \mathrm{~Hz}, \mathrm{Cy}\right), 1.26\left(\mathrm{t}, 3 \mathrm{H}, J=7.3 \mathrm{~Hz},-\mathrm{OCH}_{2} \mathrm{CH}_{3}\right)$, $1.11(\mathrm{~d}, 3 \mathrm{H}, J=6.7 \mathrm{~Hz}, i$-Pr), 1.08 (s, 9H, t-butyl), 0.91 (d. $3 \mathrm{H}, J=6.7 \mathrm{~Hz}, i-\mathrm{Pr}$ ).

${ }^{13} \mathrm{C}$ NMR $\left(125 \mathrm{MHz}, \mathrm{CDCl}_{3}\right): \delta 167.7,165.4,155.3,135.1,134.0,132,8,131.9,127,1$, $115.6,61.2,41.4,37.1,27.5,22.5,22.1,14.1$.

HRMS: EIMS $\left(\mathrm{M}^{+}\right)$calcd for $\mathrm{C}_{22} \mathrm{H}_{32} \mathrm{~N}_{2} \mathrm{O}_{5} 404.2311$, found 404.2321

\section{$\underline{{ }^{1} \text { H-NMR (Compound 32) }}$}

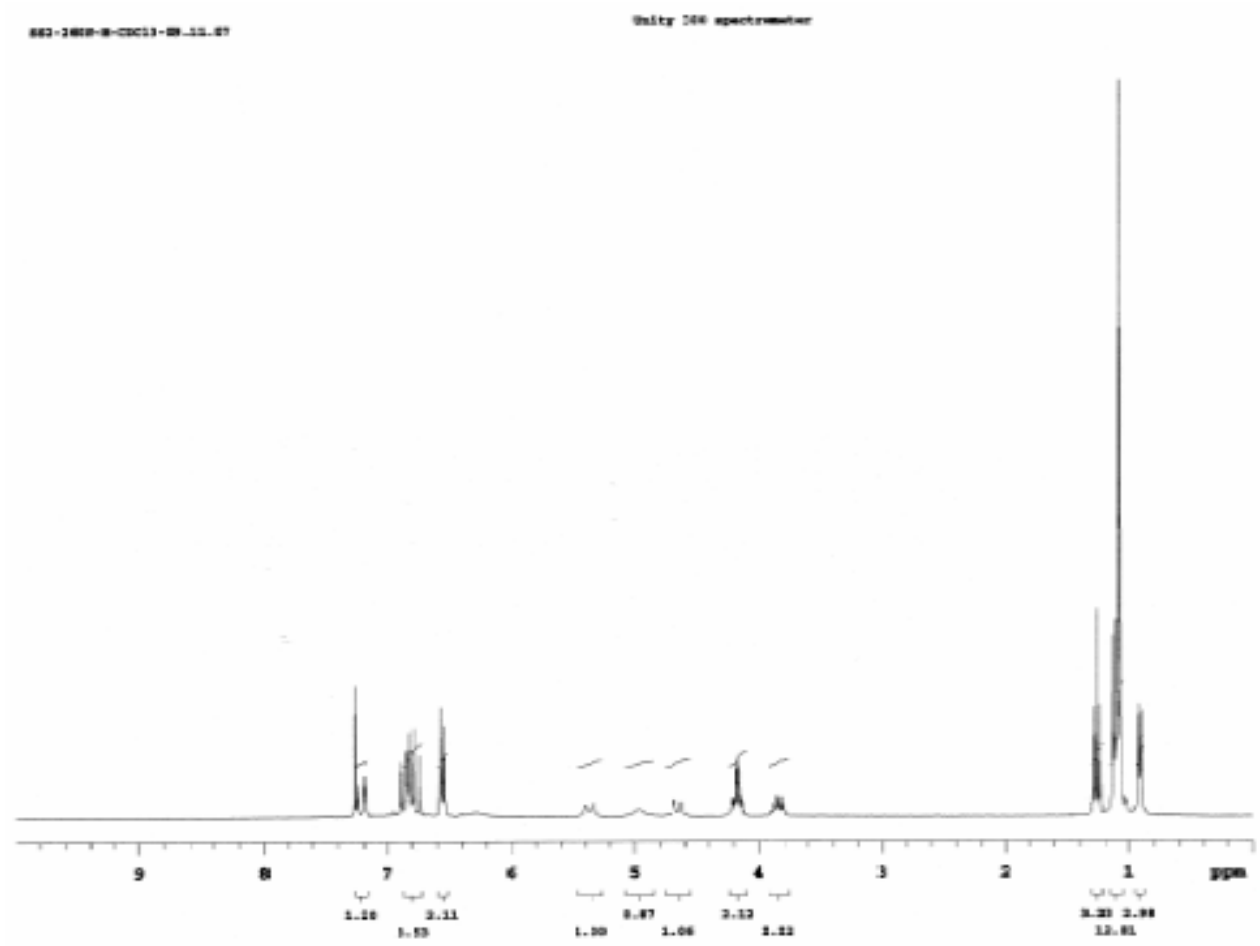




\section{${ }^{13}$ C-NMR (Compound 32)}

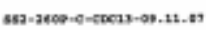

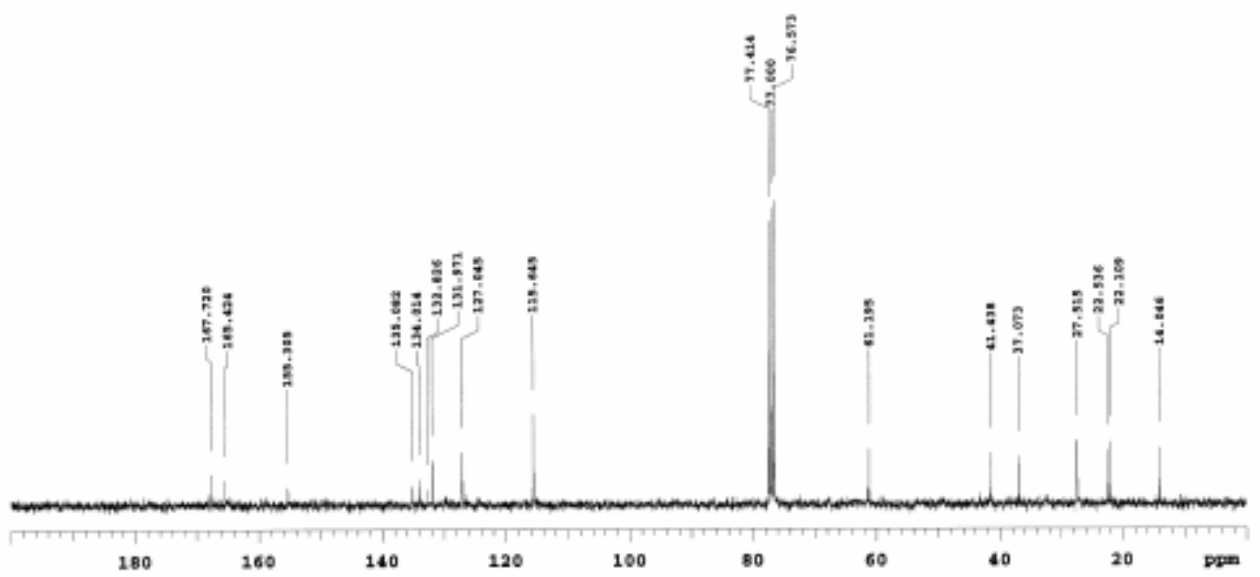

Substituted 2-azaspiro[4.5]deca-6,9-diene-3,8-diones (33)

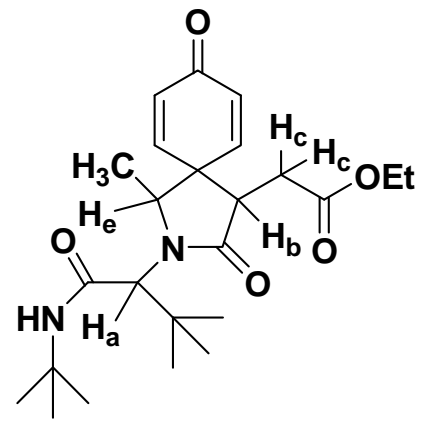

33

${ }^{1} \mathrm{H}$ NMR $\left(500 \mathrm{MHz}, \mathrm{CDCl}_{3}\right): \delta$ 6.83-7.3 (m, 2H), $6.55(\mathrm{~m}, 2 \mathrm{H}), 5.65(\mathrm{~d}, 1 \mathrm{H}, J=7.3 \mathrm{~Hz},-$ $\mathrm{N} \underline{\mathrm{H}}), 4.34\left(\mathrm{~s}, 1 \mathrm{H}, \mathrm{H}_{\mathrm{a}}\right), 4.23\left(\mathrm{~d}, 1 \mathrm{H}, J=10.4 \mathrm{~Hz}, \mathrm{H}_{\mathrm{e}}\right), 4.01-4.16\left(\mathrm{~m}, 3 \mathrm{H}, \mathrm{H}_{\mathrm{d}}\right.$ and OCH $2 \mathrm{CH} 3$ overlap), $3.54\left(\mathrm{~m}, 1 \mathrm{H}, \mathrm{H}_{\mathrm{b}}\right), 3.2\left(\mathrm{~m}, \mathrm{H}_{\mathrm{c}}\right), 2.64\left(\mathrm{~m}, 1 \mathrm{H}, \mathrm{H}_{\mathrm{e}}\right), 1.36(\mathrm{~s}, 9 \mathrm{H}, t-$ butyl), 1.27-1.36 (m, 6H, - $\underline{\mathrm{CH}}_{3}$ and $-\mathrm{OCH}_{2} \underline{\mathrm{C}}_{3}$ overlap), 0.98 (s, 9H, t-butyl).

HRMS: EIMS $\left(\mathrm{M}^{+}\right)$calcd for $\mathrm{C}_{24} \mathrm{H}_{36} \mathrm{~N}_{2} \mathrm{O}_{5} 432.2624$, found 432.2621 


\section{$\underline{{ }^{1} \text { H-NMR (Compound 33) Mixture of Diastereomers }}$}

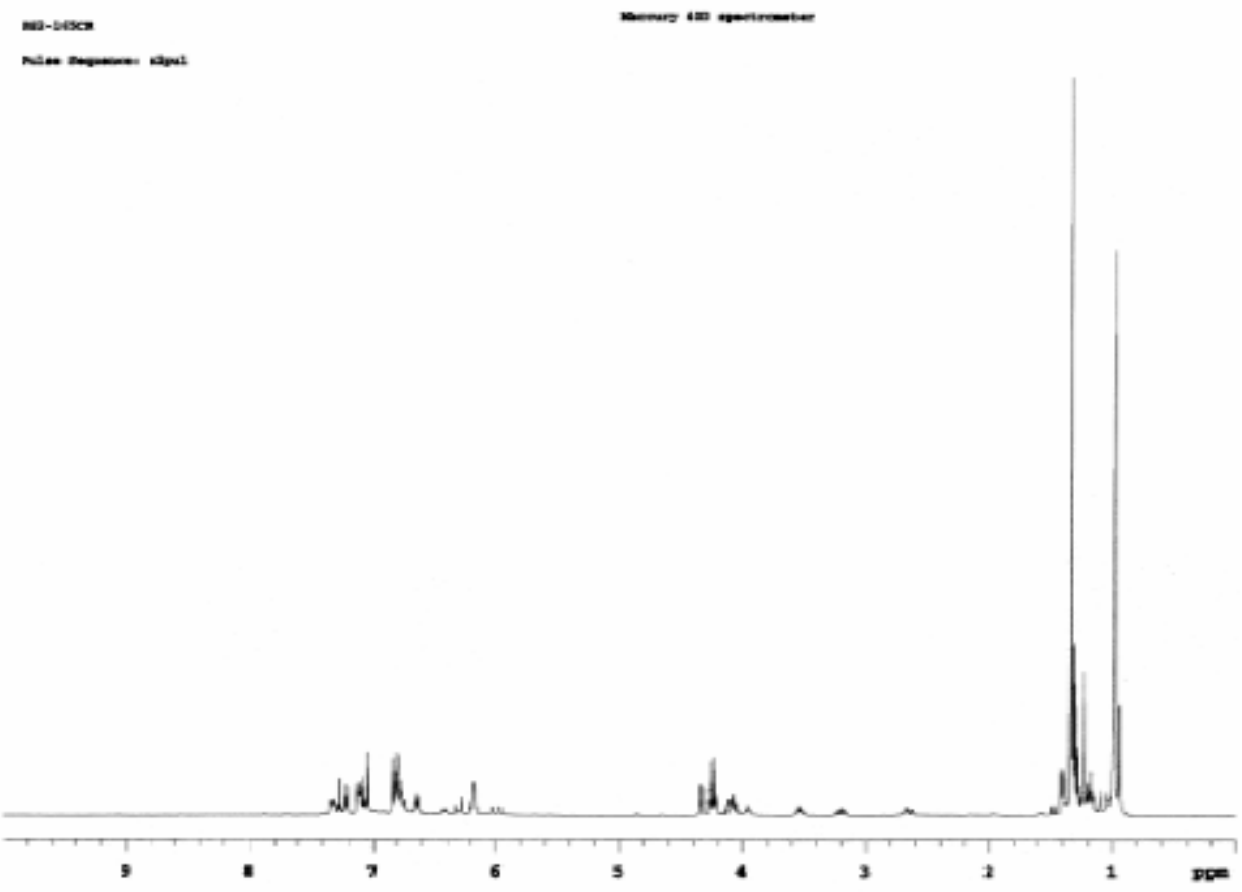

(E)-ethyl 4-((1-(tert-butylamino)-3,3-dimethyl-1-oxobutan-2-yl)(1-(4-hydroxyphenyl)ethyl)amino)-4oxobut-2-enoate (34)

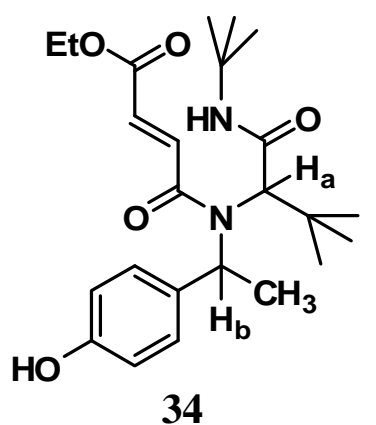

${ }^{1} \mathrm{H}$ NMR (400 MHz, $\mathrm{CDCl}_{3}$ ): $\delta 9.27$ (bs, $\left.1 \mathrm{H},-\mathrm{OH}\right), 8.44$ (bs, $\left.1 \mathrm{H},-\mathrm{N} \underline{\mathrm{H}}\right), 7.52(\mathrm{~d}, 1 \mathrm{H}, J=$ $15.4 \mathrm{~Hz},-\mathrm{C} \underline{\mathrm{H}}=\mathrm{CH}-), 7.01$ (d, $2 \mathrm{H}, J=8.1 \mathrm{~Hz}$, aryl), 6.83 (d, $1 \mathrm{H}, J=15.4 \mathrm{~Hz},-\mathrm{CH}=\mathrm{C} \underline{\mathrm{H}}-$ ), $6.63\left(\mathrm{~d}, 2 \mathrm{H}, J=8.3 \mathrm{~Hz}\right.$, aryl), $5.11\left(\mathrm{q}, 1 \mathrm{H}, J=6.5 \mathrm{~Hz}, \mathrm{H}_{\mathrm{b}}\right), 4.28(\mathrm{q}, 2 \mathrm{H}, J=7.3 \mathrm{~Hz}$, $\left.\mathrm{OC}_{2} \mathrm{CH}_{3}\right), 3.29\left(\mathrm{~s}, 1 \mathrm{H}, \mathrm{H}_{\mathrm{a}}\right), 1.61\left(\mathrm{~d}, 3 \mathrm{H}, J=6.5 \mathrm{~Hz},-\underline{\mathrm{C}}_{3}\right), 1.33(\mathrm{t}, 3 \mathrm{H}, J=7.3 \mathrm{~Hz},-$ $\mathrm{OCH}_{2} \mathrm{CH}_{3}$ ), 1.22 (s, 9H, t-butyl), 1.13 (s, 9H, t-butyl).

${ }^{13} \mathrm{C}$ NMR $\left(100 \mathrm{MHz}, \mathrm{CDCl}_{3}\right): \delta 170.6,167.8,165.3,157.6,136.2,131.5,128.8,126.8$, $116.0,72.4,61.4,58.1,51.1,35.1,29.7,28.6,17.2,14.1$.

HRMS: EIMS $\left(\mathrm{M}^{+}\right)$calcd for $\mathrm{C}_{24} \mathrm{H}_{36} \mathrm{~N}_{2} \mathrm{O}_{5} 432.2624$, found 432.2621 
${ }^{1}$ H-NMR (Compound 34)

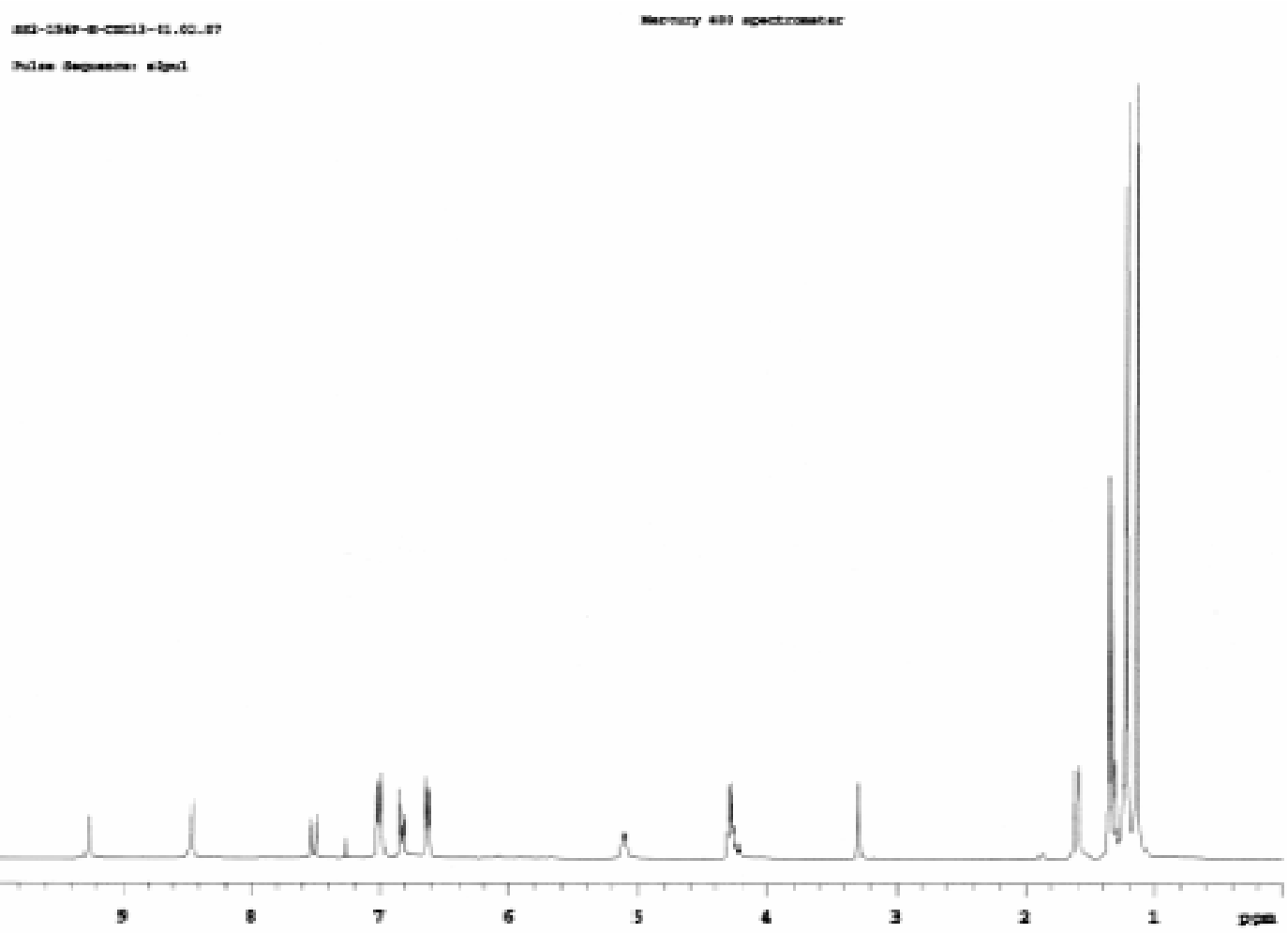

${ }^{13}$ C-NMR (Compound 34)

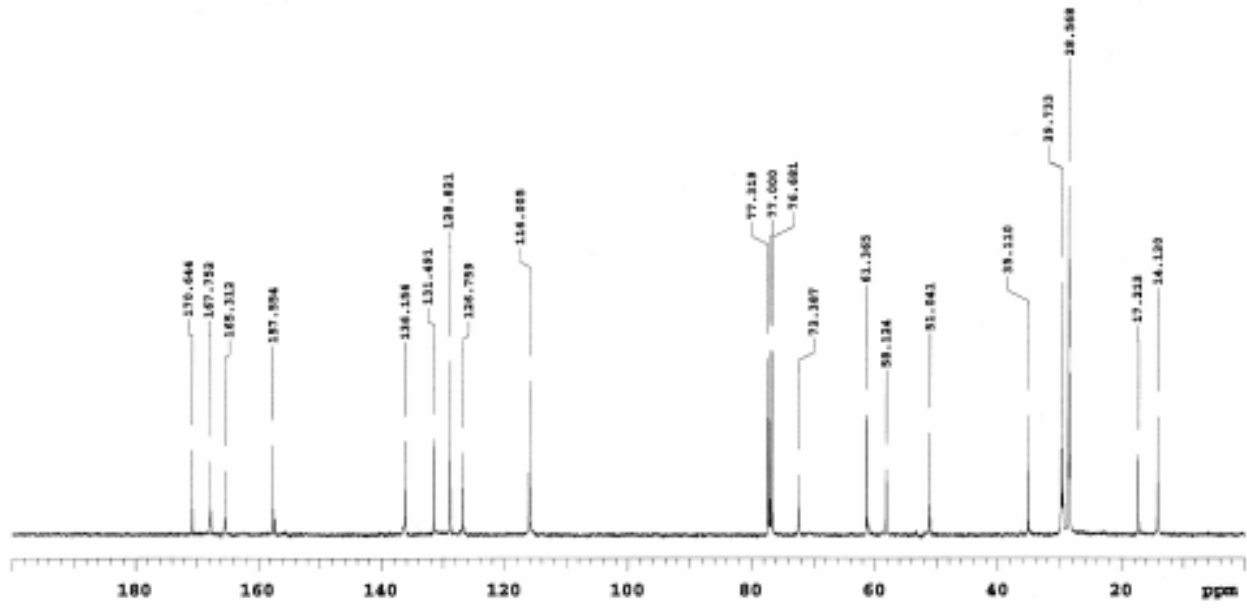




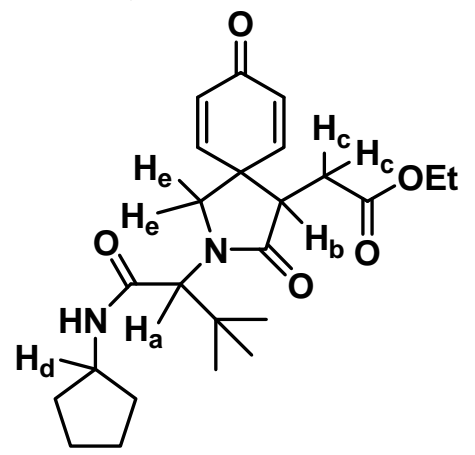

35

${ }^{1} \mathrm{H}$ NMR (400 MHz, $\left.\mathrm{CDCl}_{3}\right): \delta 6.89(\mathrm{~m}, 2 \mathrm{H}), 6.36(\mathrm{~m}, 2 \mathrm{H}), 5.94(\mathrm{~d}, 1 \mathrm{H}, J=7.3 \mathrm{~Hz},-\mathrm{N} \underline{\mathrm{H}}$ ), $4.28\left(\mathrm{~s}, 1 \mathrm{H}, \mathrm{H}_{\mathrm{a}}\right), 4.12-4.18\left(\mathrm{~m}, 1 \mathrm{H}, \mathrm{H}_{\mathrm{d}}\right), 4.07\left(\mathrm{q}, 2 \mathrm{H}, J=7.3 \mathrm{~Hz},-\mathrm{OC}_{2} \mathrm{CH}_{3}\right), 3.70$ (dd, $\left.2 \mathrm{H}, J=11.4,14.6 \mathrm{~Hz}, \mathrm{H}_{\mathrm{e}}\right), 3.30\left(\mathrm{dd}, 1 \mathrm{H}, J=6.5,8.1 \mathrm{~Hz}, \mathrm{H}_{\mathrm{b}}\right), 2.67(\mathrm{dd}, 1 \mathrm{H}, J=6.1,16.7$ $\left.\mathrm{Hz}, \mathrm{H}_{\mathrm{c}}\right), 2.12\left(\mathrm{dd}, 1 \mathrm{H}, J=7.3,16.2 \mathrm{~Hz}, \mathrm{H}_{\mathrm{c}}\right), 1.88-2.23(\mathrm{~m}, 2 \mathrm{H}, \mathrm{Cp}), 1.51-1.72(\mathrm{~m}, 4 \mathrm{H}$, Cp), 1.29-1.44 (m, 2H, Cp), 1.20 (t, 3H, $\left.J=7.3 \mathrm{~Hz},-\mathrm{OCH}_{2} \mathrm{CH}_{3}\right), 1.05$ (s, 9H, t-Bu).

${ }^{13} \mathrm{C}$ NMR $\left(100 \mathrm{MHz}, \mathrm{CDCl}_{3}\right): \delta 185.1,173.3,171.4,168.2,149.3,145.9,131.5,130.9$, $63.1,61.1,52.6,51.2,47.6,47.0,35.1,33.0,32.9,30.4,27.8,27.6,23.7,14.0$.

HRMS: EIMS $\left(\mathrm{M}^{+}\right)$calcd for $\mathrm{C}_{24} \mathrm{H}_{34} \mathrm{~N}_{2} \mathrm{O}_{5} 430.2468$, found 430.2475.

\section{$\underline{{ }^{1} \text { H-NMR (Compound 35) Mixture of Diastereomers }}$}

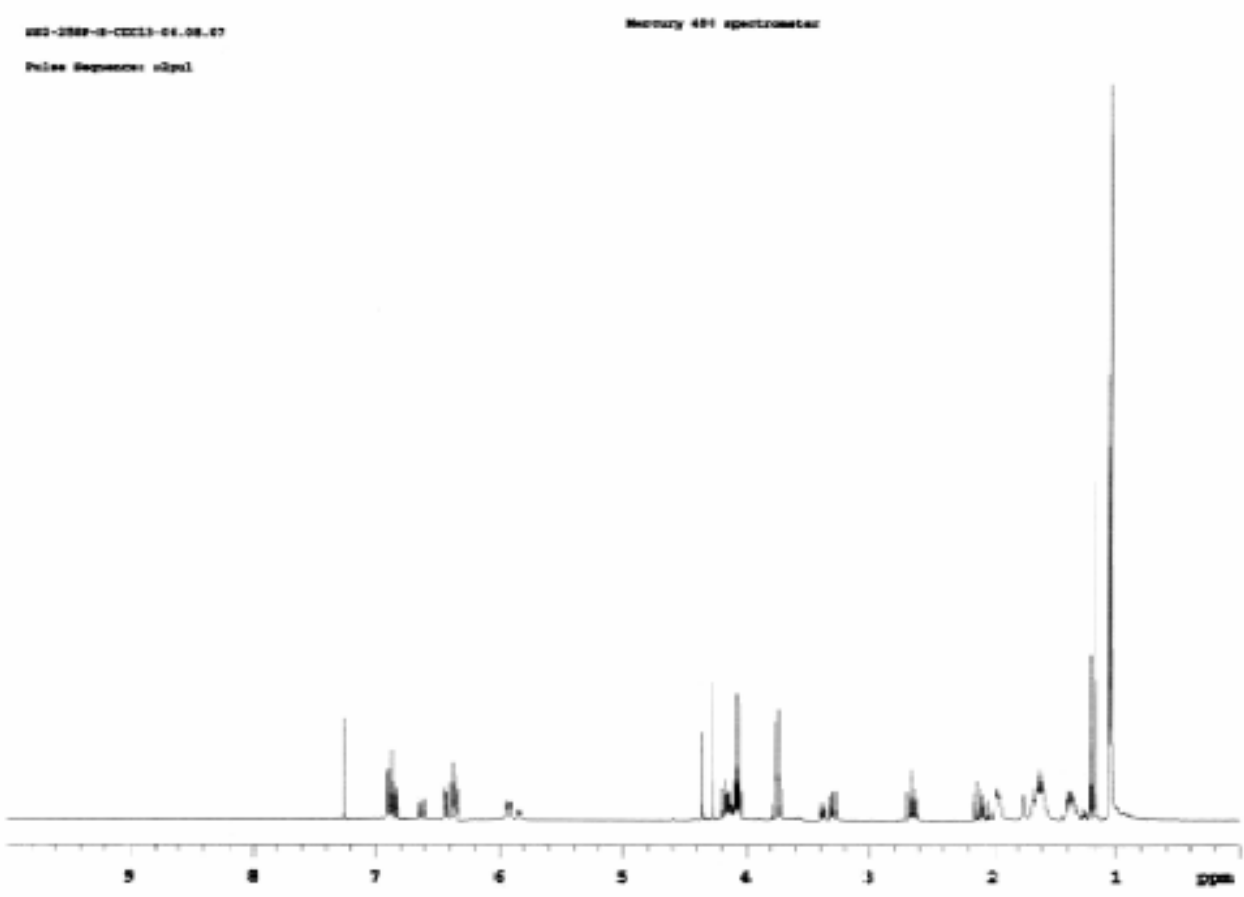




\section{${ }^{13}$ C-NMR (Compound 35) Mixture of Diastereomers}
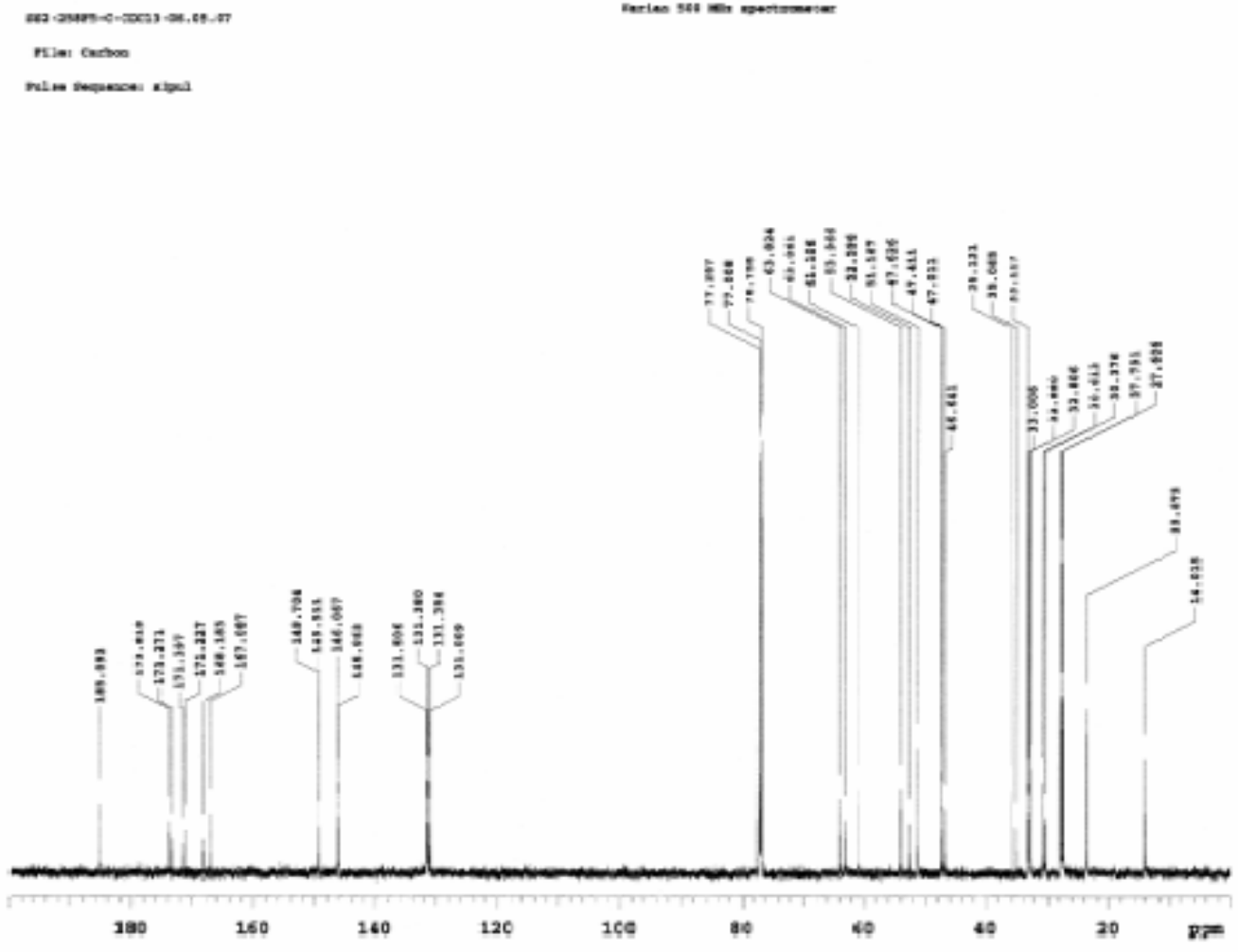

(E)-ethyl 4-((1-(cyclopentylamino)-3,3-dimethyl-1-oxobutan-2-yl)(4-hydroxybenzyl)amino)-4-oxobut2-enoate (36)<smiles>CCOC(=O)/C=C/C(=O)N(Cc1ccc(O)cc1)C(C)(C(=O)NC1CCCC1)C(C)(C)C</smiles>

36

${ }^{1} \mathrm{H}$ NMR (400 MHz, $\left.\mathrm{CDCl}_{3}\right): \delta 7.51$ (bs, $\left.1 \mathrm{H},-\mathrm{OH}\right), 7.21(\mathrm{~d}, 1 \mathrm{H}, J=14.6 \mathrm{~Hz},-\mathrm{C} \underline{\mathrm{H}}=\mathrm{CH}-$ ),

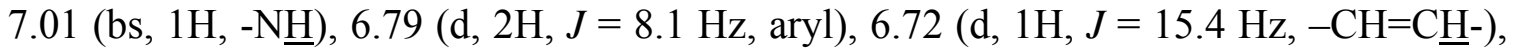
$6.55(\mathrm{~d}, 2 \mathrm{H}, J=8.1 \mathrm{~Hz}$, aryl), 5.35 (bs, $1 \mathrm{H}, \mathrm{Bn}), 5.02\left(\mathrm{bs}, 1 \mathrm{H}, \mathrm{H}_{\mathrm{a}}\right), 4.64(\mathrm{~d}, 1 \mathrm{H}, J=\overline{16} .2$ $\mathrm{Hz}, \mathrm{Bn}), 4.09-4.23\left(\mathrm{~m}, 2 \mathrm{H},-\mathrm{OCH}_{2} \mathrm{CH}_{3}\right), 3.87-3.97$ (m, 1H, Cp), 1.82-1.94 (m, 1H, Cp), 1.31-1.76 (m, 7H, Cp), 1.24 (t, 3H, $\left.J=7.3 \mathrm{~Hz},-\mathrm{OCH}_{2} \mathrm{CH}_{3}\right), 1.07$ (s, 9H, t-butyl)

${ }^{13} \mathrm{C}$ NMR $\left(100 \mathrm{MHz}, \mathrm{CDCl}_{3}\right): \delta 168.4,167.6,165.4,155.6,134.2,131.7,130.2,126.8$, $115.6,65.8,61.2,51.1,37.0,32.8,32.3,27.5,23.7,14.1$.

HRMS: EIMS $\left(\mathrm{M}^{+}\right)$calcd for $\mathrm{C}_{24} \mathrm{H}_{34} \mathrm{~N}_{2} \mathrm{O}_{5} 430.2468$, found 430.2462 
$\underline{{ }^{1} \text { H-NMR (Compound 36) }}$

m-2uce

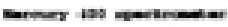

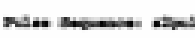

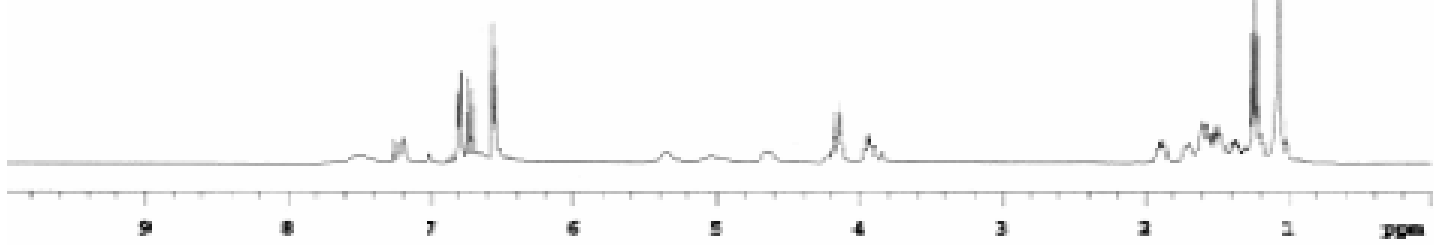

${ }^{13}$ C-NMR (Compound 36)

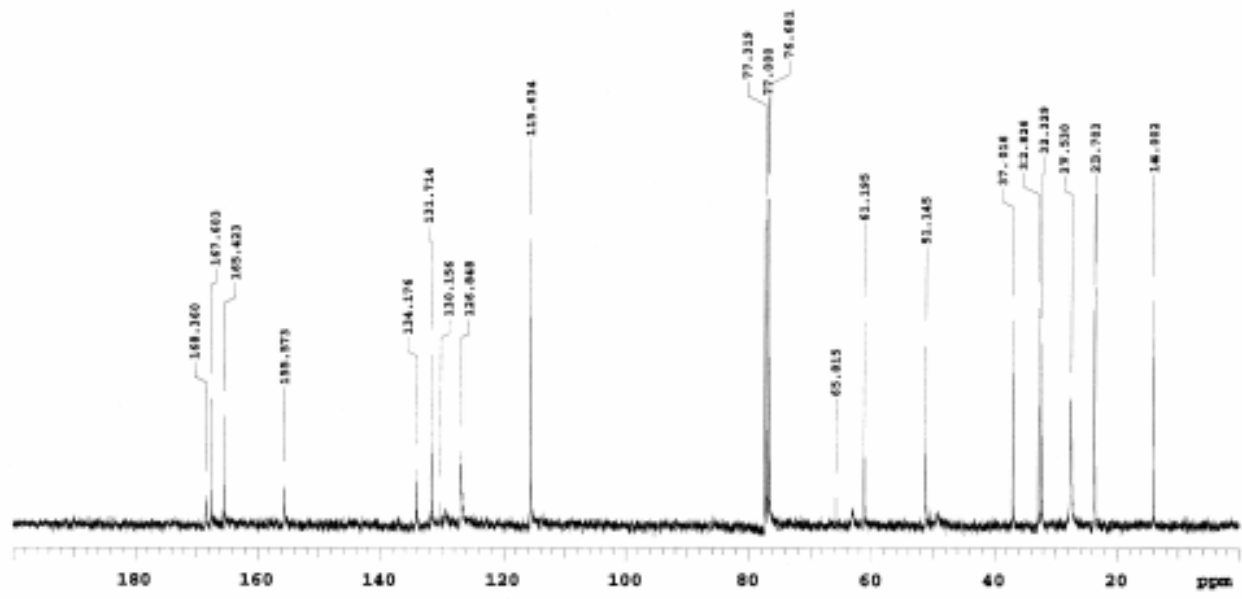




\section{Modified General Procedure 1 for Aprotic Solvent (Starting with four separate}

substrates). In a $10 \mathrm{~mL}$ vial (CEM Discover System) equipped with a magnetic stirring bar, $p$-methoxybenzylamine (15 mg, $0.14 \mathrm{mmol})$, thiophene-2-carboxyaldehyde (12 $\mathrm{mg}$, $0.14 \mathrm{mmol})$, fumaric acid monoethyl ester $(25 \mathrm{mg}, 0.14 \mathrm{mmol})$ and benzyl isocyanide (17 $\mu \mathrm{L}, 0.14 \mathrm{mmol}$ ) were added to $1.5 \mathrm{~mL}$ of methylene chloride. The vial was capped properly and placed in the microwave. The microwave was then run at two stages: (a) 1 hr at $50{ }^{\circ} \mathrm{C}$, 10 Bar with $300 \mathrm{~W}$ and (b) 20-25 min, $200{ }^{\circ} \mathrm{C}, 18$ Bar with $300 \mathrm{~W}$. After cooling the vial to room temperature, water was added and the mixture was shaken thoroughly. The organic layer was separated and the aqueous layer was extracted with methylene chloride $(2 \times 2 \mathrm{~mL})$. The organic layers were combined and washed with saturated $\mathrm{NaHCO}_{3}(3 \times 3 \mathrm{~mL}), 1 \mathrm{~N} \mathrm{HCl}(3 \times 3 \mathrm{~mL})$, and brine $(3 \times 3 \mathrm{~mL})$. The organic layer was separated. All aqueous layers were combined and extracted with $3 \mathrm{~mL}$ of methylene chloride. The organic layers were combined, dried with $\mathrm{Na}_{2} \mathrm{SO}_{4}$ and filtered. The filtrate was concentrated under vacuum and the residue was purified by silica gel column chromatography using a 1:2 mixture of ethyl acetate and hexane as the eluent.

\section{Modified General Procedure 2 for Aprotic Solvent (Starting from a preformed} acyclic Ugi product). To a $10 \mathrm{~mL}$ vial (CEM Discover System) equipped with magnetic stir bar under argon, $50 \mathrm{mg}$ of acyclic Ugi product was added along with $2.5 \mathrm{~mL}$ of dry $\underline{\mathrm{CH}}_{2} \underline{\mathrm{Cl}}_{2}$. The vial was capped properly and placed in the microwave. Then the microwave was run for $20-25$ min at $200{ }^{\circ} \mathrm{C}$, 18 Bar with $300 \mathrm{~W}$. After cooling the vial to room temperature, ethyl acetate was added and the mixture was shaken thoroughly. The organic layer was separated and the aqueous layer was extracted with ethyl acetate (2 x $2 \mathrm{~mL}$ ). The organic layers were combined, dried over $\mathrm{Na}_{2} \mathrm{SO}_{4}$ and filtered. The filtrate was concentrated under vacuum and purified by silica gel column chromatography using 1:2 mixture of ethyl acetate and hexane as eluent. 
Thiophene-derived tricyclic lactam (37)

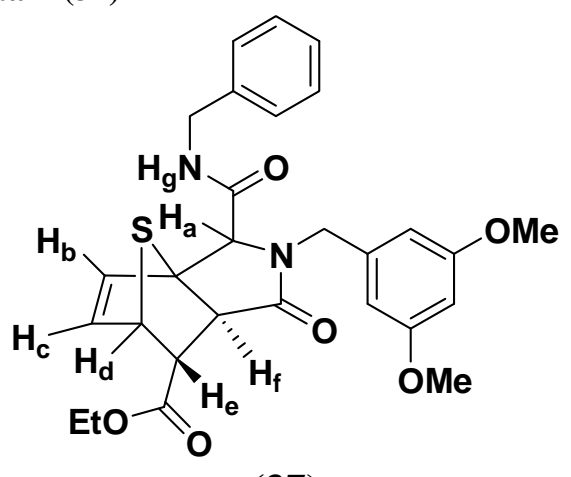

(37)

${ }^{1} \mathrm{H}$ NMR (400 MHz, $\left.\mathrm{CDCl}_{3}\right): \delta$ 7.19-7.42 (m, 7H, aryl), $6.82(\mathrm{~m}, 1 \mathrm{H}$, aryl), $6.47(\mathrm{~d}, 1 \mathrm{H}, J$ $\left.=6.2 \mathrm{~Hz}, \mathrm{H}_{\mathrm{b}}\right), 6.32\left(1 \mathrm{H}, \mathrm{H}_{\mathrm{c}}\right), 6.25\left(\mathrm{br}, 1 \mathrm{H}, \mathrm{H}_{\mathrm{g}}\right), 5.03(\mathrm{~d}, 1 \mathrm{H}, J=14.7 \mathrm{~Hz}, \mathrm{Bn}), 4.41-4.46$ (m, 3H, Bn and $\mathrm{H}_{\mathrm{d}}$ overlap), $4.17\left(\mathrm{~m}, 2 \mathrm{H},-\mathrm{OCH}_{2} \mathrm{CH}_{3}\right), 3.99\left(\mathrm{~s}, 1 \mathrm{H}, \mathrm{H}_{\mathrm{a}}\right), 3.94(\mathrm{~m}, 1 \mathrm{H}, \mathrm{Bn}$ and $\mathrm{H}_{\mathrm{e}}$ overlap), 3.85 (d, 1H, $\mathrm{H}_{\mathrm{e}}$ and Bn overlap), 3.78 (s, 6H, -OC$\left.\underline{\mathrm{H}}_{3}\right), 3.42$ (d, 1H, J = $\left.3.6 \mathrm{~Hz}, \mathrm{H}_{\mathrm{f}}\right), 1.25$ (t, $\left.3 \mathrm{H}, J=7.1 \mathrm{~Hz}, \mathrm{CH}_{3}\right)$.

\section{${ }^{1}$ H-NMR (Compound 37) Mixture of Diastereomers}

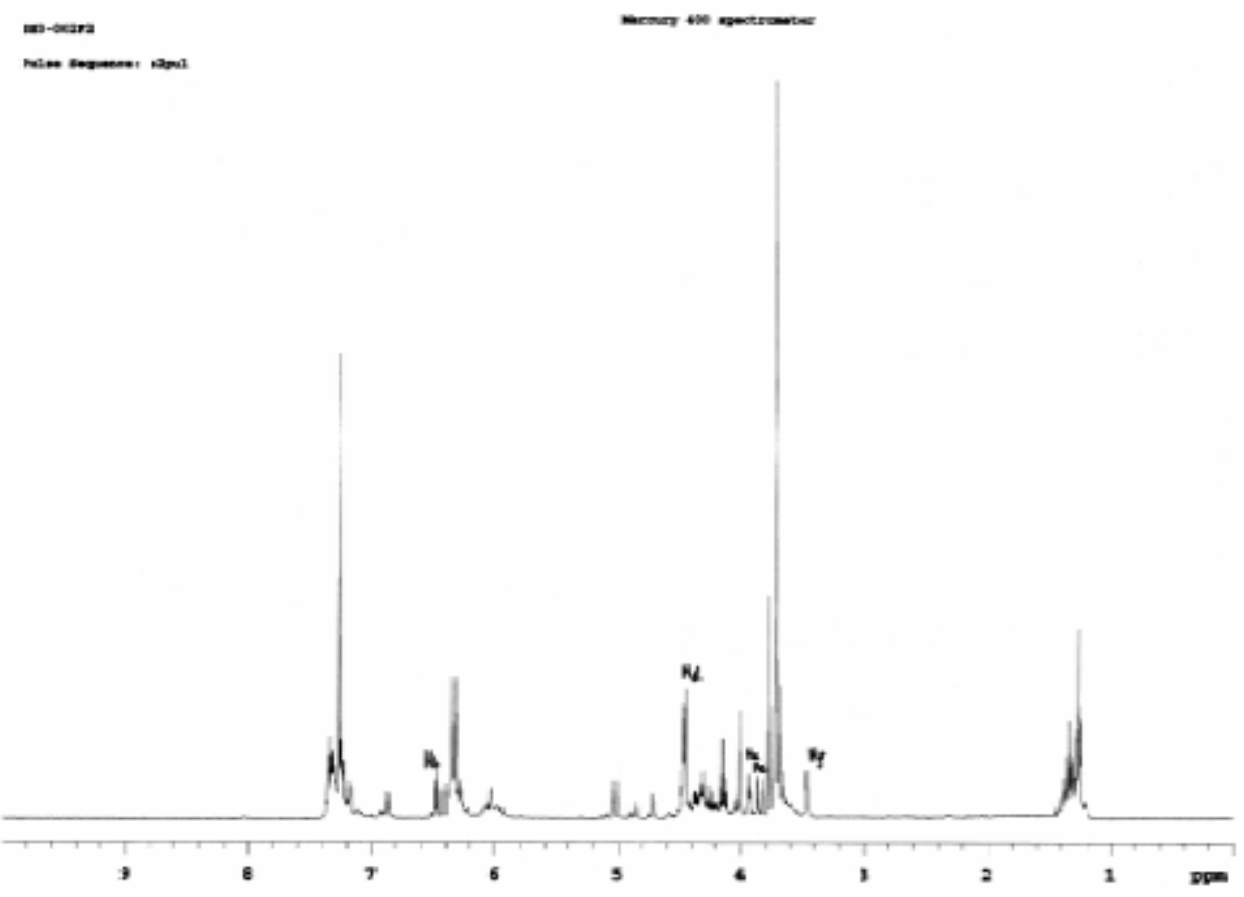


Thiophene-derived tricyclic lactam (38)

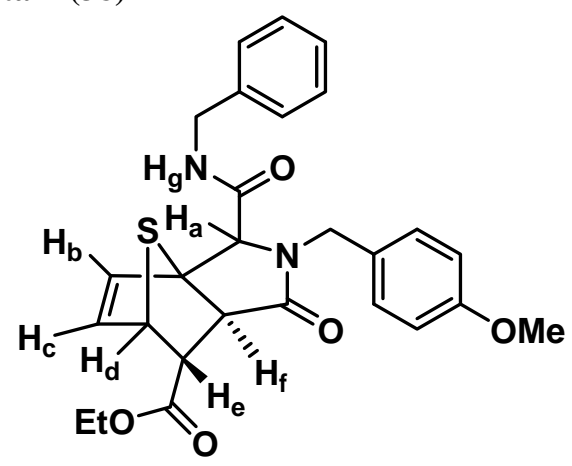

(38)

${ }^{1} \mathrm{H}$ NMR $\left(500 \mathrm{MHz}, \mathrm{CDCl}_{3}\right)$ : $\delta$ 7.28-7.38 (m, 3H, aryl), 7.19-7.24 (m, 2H, aryl), $7.10(\mathrm{~d}$, $2 \mathrm{H}, J=8.6 \mathrm{~Hz}$, aryl), $6.81\left(\mathrm{~d}, 2 \mathrm{H}, J=8.6 \mathrm{~Hz}\right.$, aryl), $6.48\left(\mathrm{~d}, 1 \mathrm{H}, J=6.1 \mathrm{~Hz}, \mathrm{H}_{\mathrm{b}}\right), 6.32$ $\left(\mathrm{dd}, 1 \mathrm{H}, J=3.6,6.1 \mathrm{~Hz}, \mathrm{H}_{\mathrm{c}}\right), 6.30\left(\mathrm{brt}, 1 \mathrm{H}, \mathrm{H}_{\mathrm{g}}\right), 4.93(\mathrm{~d}, 1 \mathrm{H}, J=14.7 \mathrm{~Hz}, \mathrm{Bn}), 4.41-4.46$ (m, $3 \mathrm{H}, \mathrm{Bn}$ and $\mathrm{H}_{\mathrm{d}}$ overlap), $4.14\left(\mathrm{q}, 2 \mathrm{H}, J=7.1 \mathrm{~Hz},-\mathrm{OC}_{2} \mathrm{CH}_{3}\right), 3.98\left(\mathrm{~s}, 1 \mathrm{H}, \mathrm{H}_{\mathrm{a}}\right), 3.94$ (d, $1 \mathrm{H}, J=14.7 \mathrm{~Hz}, \mathrm{Bn}$ and $\mathrm{H}_{\mathrm{e}}$ overlap), 3.92 (t, $1 \mathrm{H}, J=\mathrm{Hz}, \mathrm{H}_{\mathrm{e}}$ and Bn overlap), 3.78 (s, $\left.3 \mathrm{H}, \mathrm{OCH}_{3}\right), 3.42\left(\mathrm{~d}, 1 \mathrm{H}, J=3.6 \mathrm{~Hz}, \mathrm{H}_{\mathrm{f}}\right), 1.25\left(\mathrm{t}, 3 \mathrm{H}, J=7.1 \mathrm{~Hz}, \mathrm{CH}_{3}\right)$.

${ }^{13} \mathrm{C}$ NMR $\left(125 \mathrm{MHz}, \mathrm{CDCl}_{3}\right): \delta 173.5,170.5,166.9,159.5,136.9,136.6,129.9,128.9$, $127.9,127.8,126.8,114.3,71.3,62.3,61.4,55.3,54.9,54.3,51.7,45.9,43.9,29.7,14.2$.

HRMS: EIMS $\left(\mathrm{M}^{+}\right)$calcd for $\mathrm{C}_{27} \mathrm{H}_{28} \mathrm{~N}_{2} \mathrm{O}_{5} \mathrm{~S} 492.1719$, found 492.1713 .

(a) ${ }^{1} \mathrm{H}$ NMR of Diels-Alder Product (38) at room temperature.
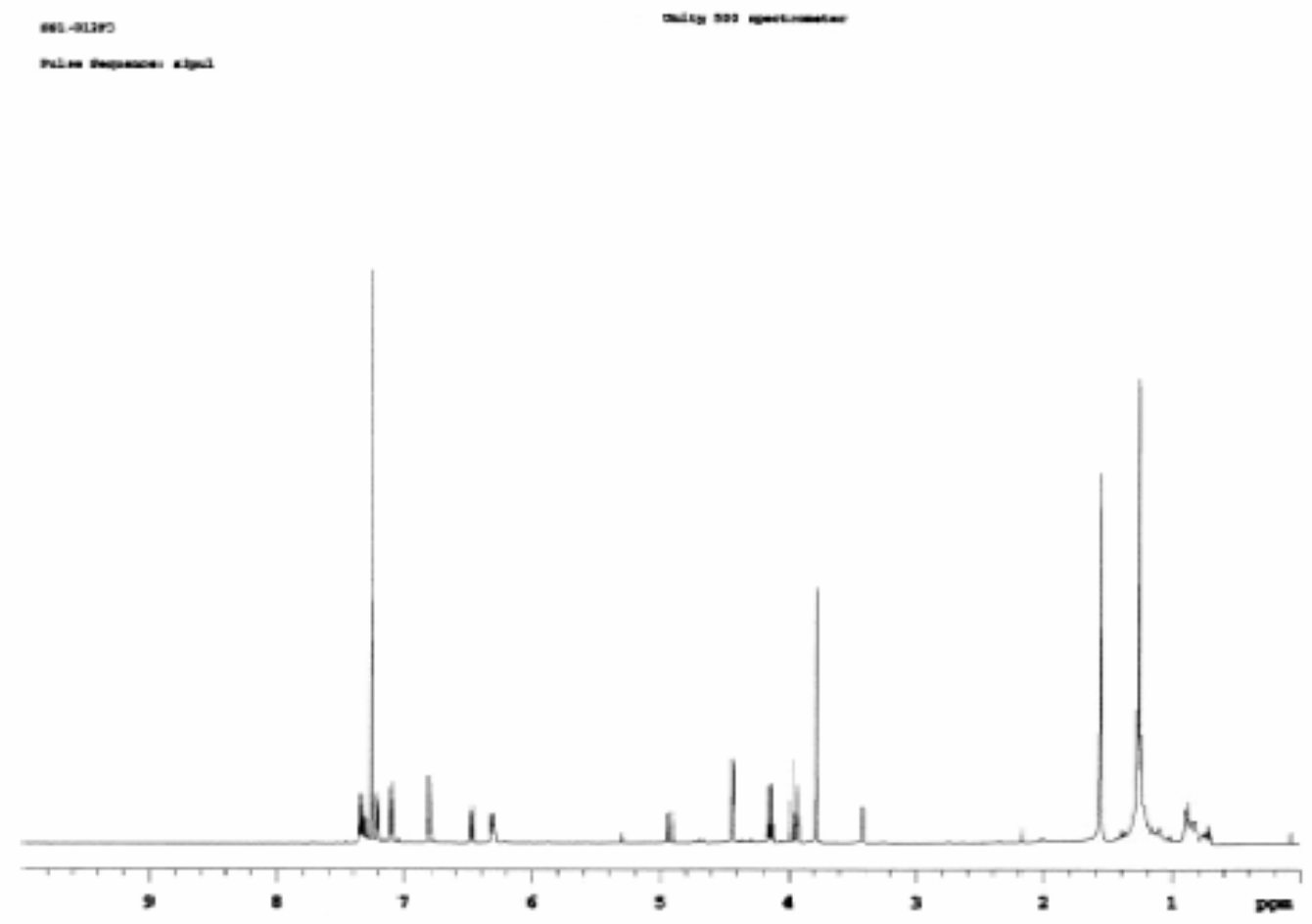
(b) ${ }^{1} \mathrm{H}$ NMR of Diels-Alder Product (38) at $40{ }^{\circ} \mathrm{C}$.
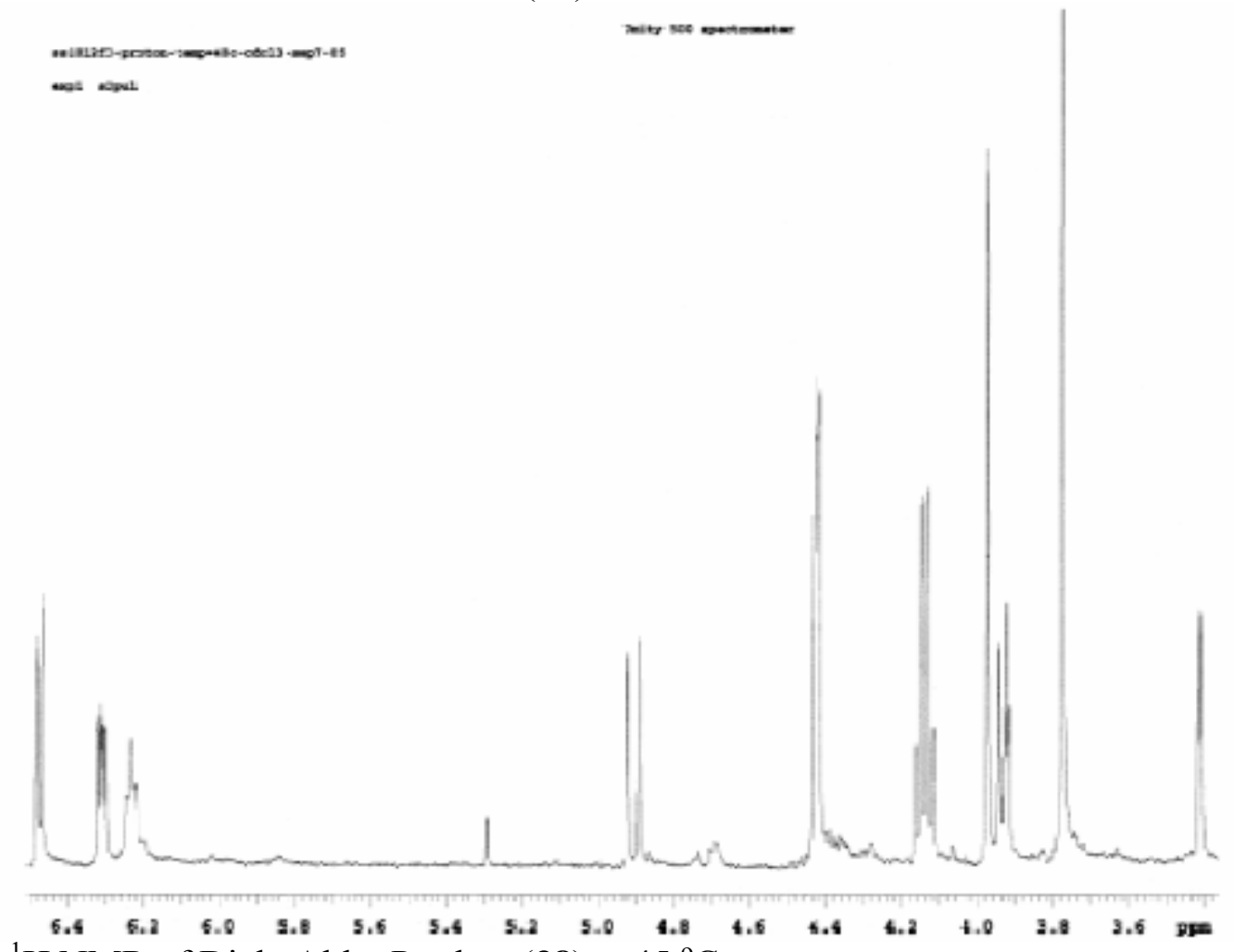

(c) ${ }^{1} \mathrm{H}$ NMR of Diels-Alder Product (38) at $45^{\circ} \mathrm{C}$.

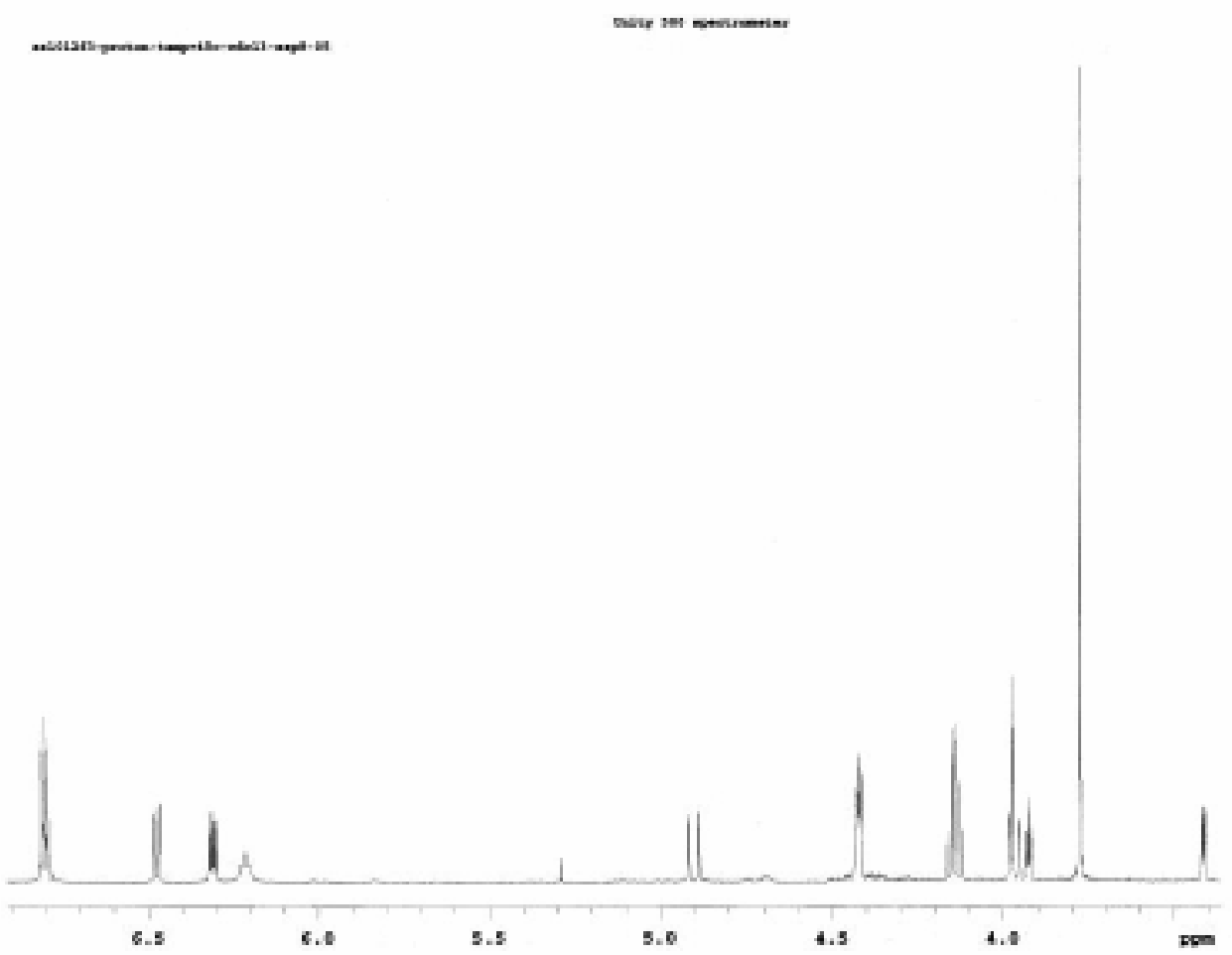


(d) ${ }^{1} \mathrm{H}$ NMR of Diels-Alder Product (38) at $48{ }^{\circ} \mathrm{C}$.

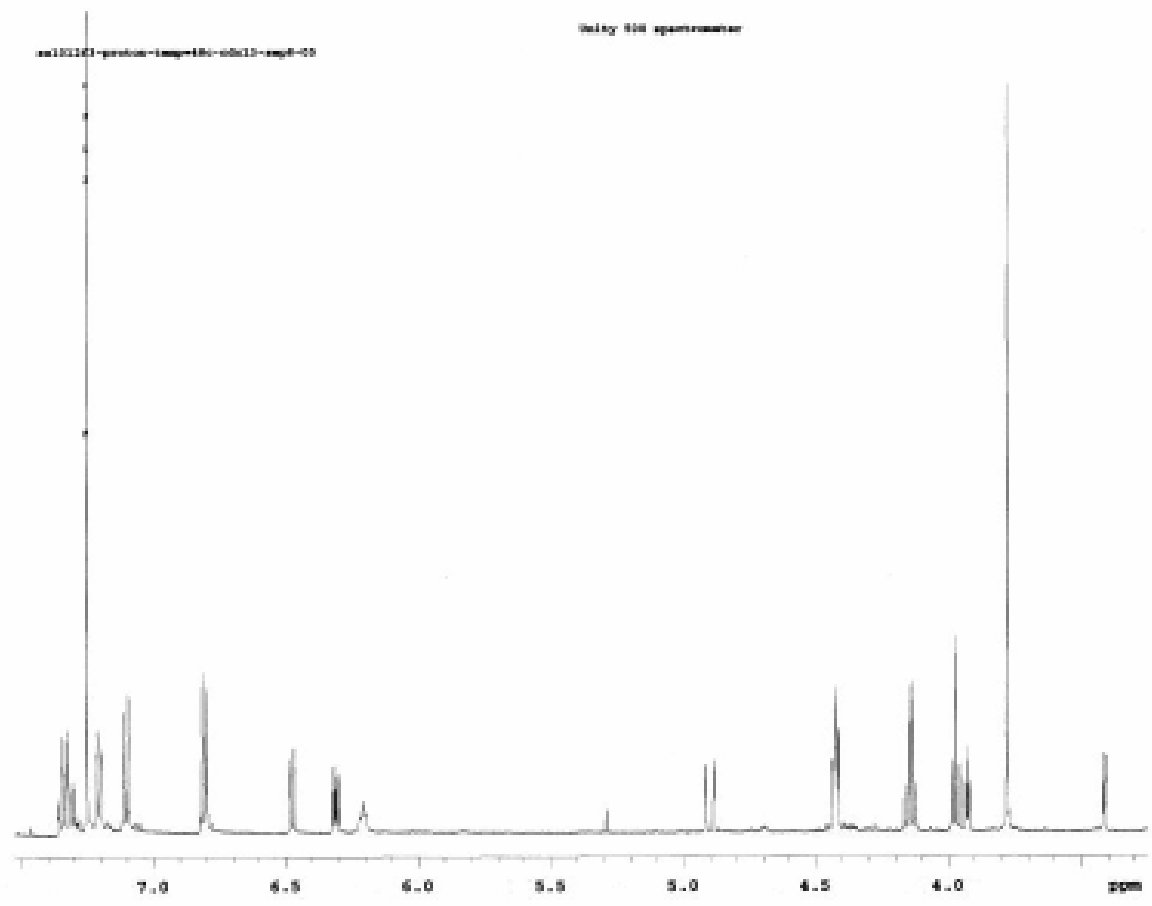

(e) ${ }^{13} \mathrm{C}$ NMR of Diels-Alder Product (38) at room temperature.

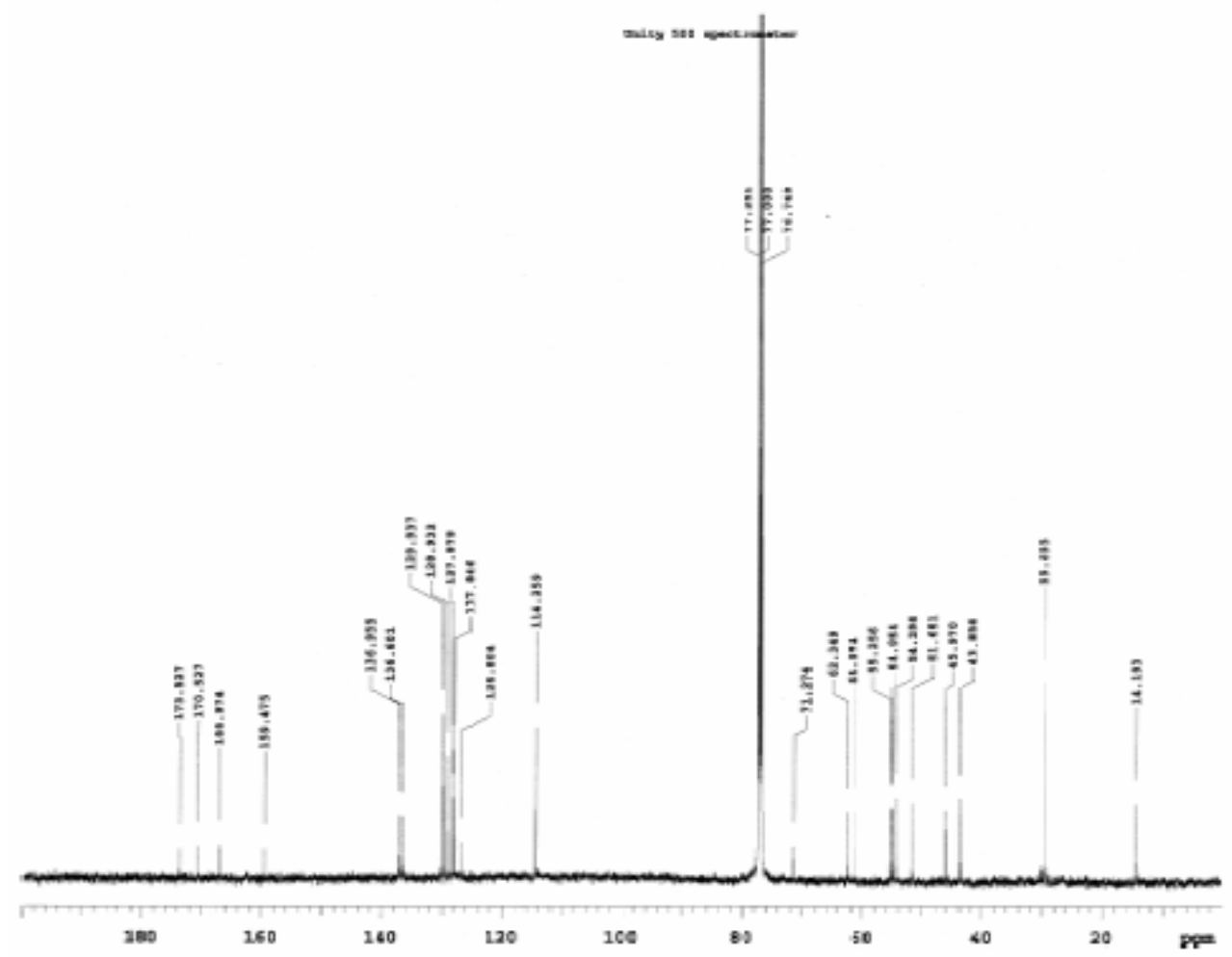


(f) gHMQC of Diels-Alder Product (38).

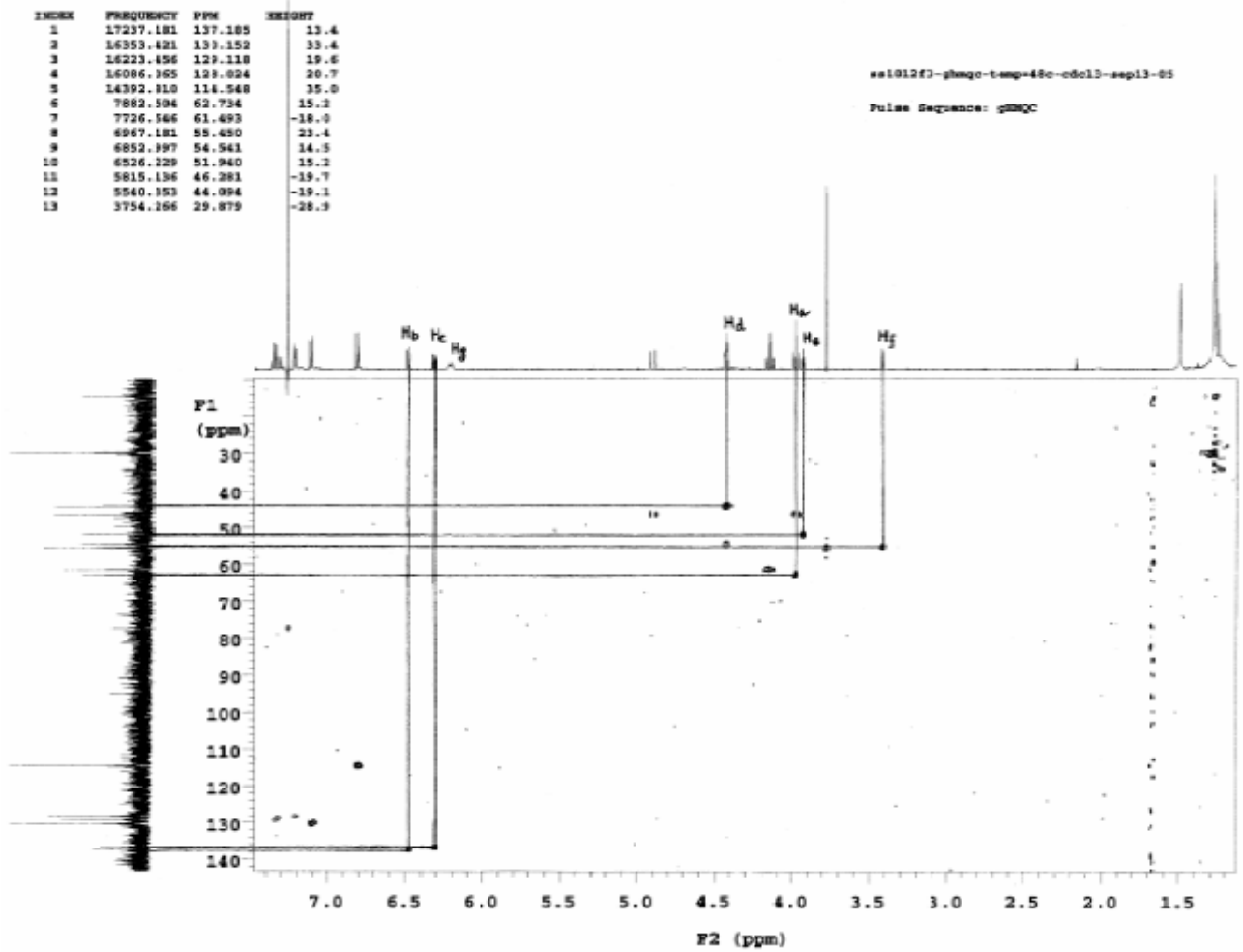

(g) gCOSY of Diels-Alder Product (38).

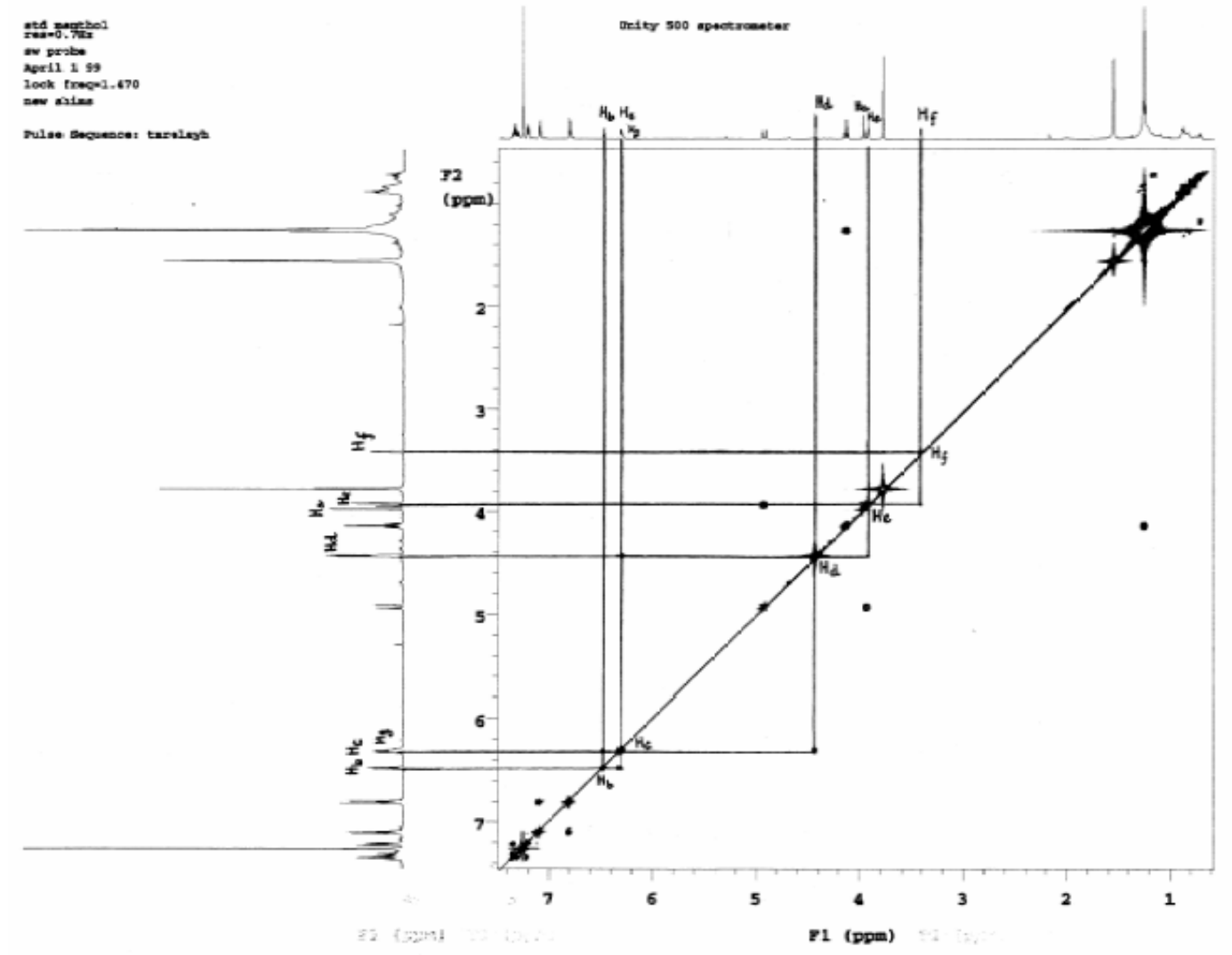


(h) gHMBC of Diels-Alder Product (38).
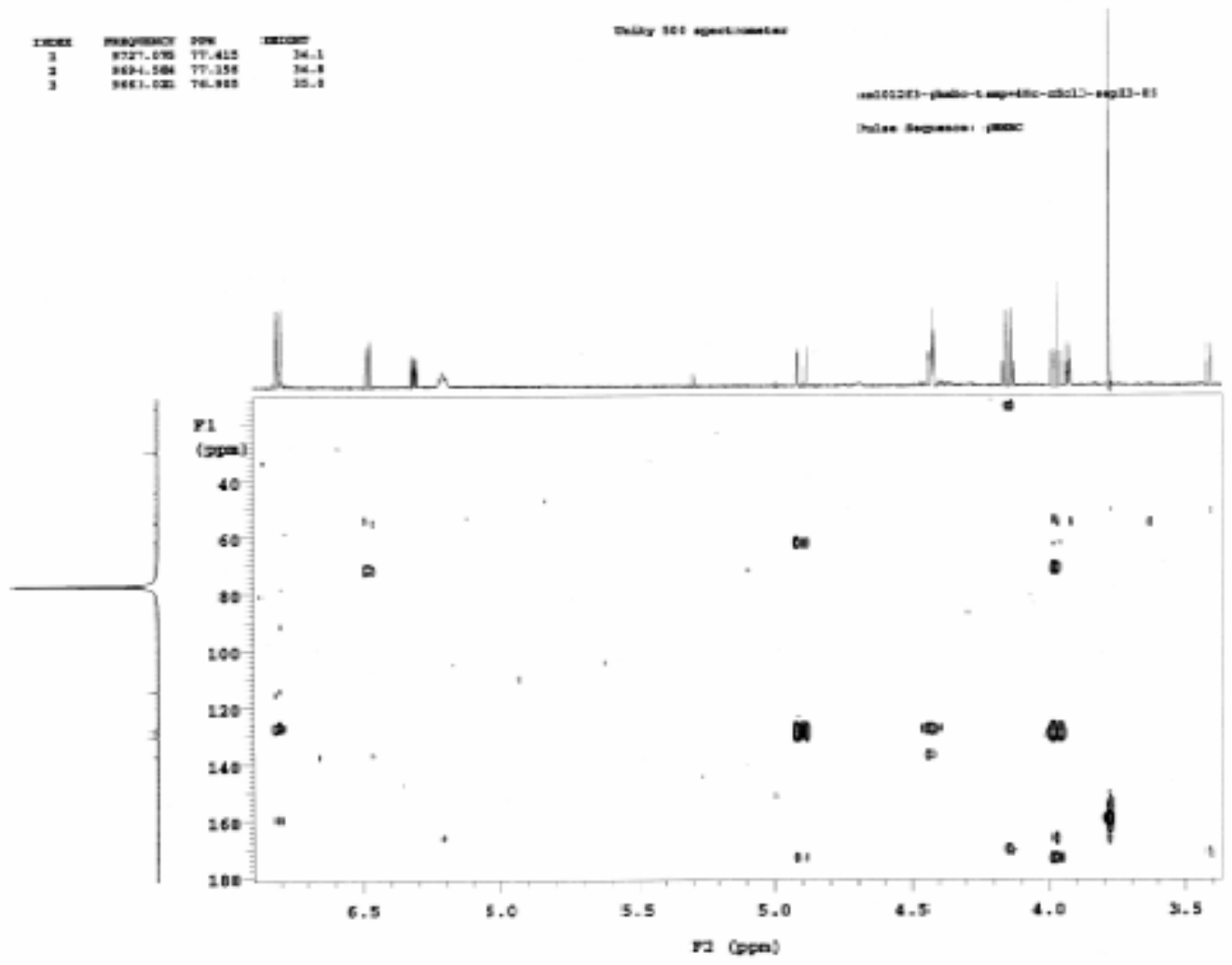

(E)-ethyl 3-(N-(1-(tert-butylcarbamoyl)-2,2-dimethylpropyl)-N-(4-ethoxybenzyl)carbamoyl)acrylate (39)

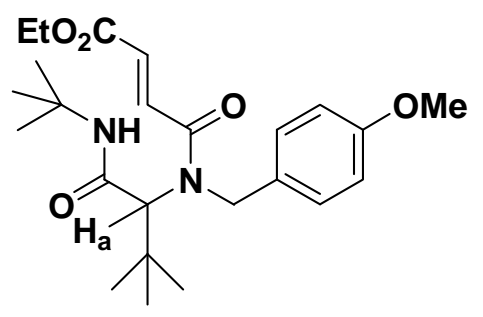

39

${ }^{1} \mathrm{H}$ NMR $\left(500 \mathrm{MHz}, \mathrm{CDCl}_{3}\right): \delta 7.18(\mathrm{~d}, 1 \mathrm{H}, J=15.2 \mathrm{~Hz},-\mathrm{C} \underline{\mathrm{H}}=\mathrm{CH}-), 7.01$ (d, 2H, $J=8.6$ $\mathrm{Hz}, \operatorname{aryl}), 6.78(\mathrm{~d}, 2 \mathrm{H}, J=8.6 \mathrm{~Hz}, \operatorname{aryl}), 6.70(\mathrm{~d}, 1 \mathrm{H}, J=15.2 \mathrm{~Hz},-\mathrm{CH}=\mathrm{CH}-), 5.77$ (bs, $1 \mathrm{H}$, $-\mathrm{NH}), 5.36(\mathrm{~d}, 1 \mathrm{H}, J=16.7 \mathrm{~Hz}, \mathrm{Bn}), 4.91$ (bs, 1H, $\left.\mathrm{H}_{\mathrm{a}}\right), 4.68$ (d, 1H, J=17.2 Hz, Bn), 4.09$4.21\left(\mathrm{~m}, 2 \mathrm{H},-\mathrm{OCH}_{2} \mathrm{CH}_{3}\right), 1.24\left(\mathrm{t}, 3 \mathrm{H}, J=7.1 \mathrm{~Hz},-\mathrm{OCH}_{2} \underline{\mathrm{CH}}_{3}\right), 1.22(\mathrm{~s}, 9 \mathrm{H}, \mathrm{N}-\mathrm{t}-\mathrm{Bu}), 1.09$ (s, 9H, C-t-Bu).

${ }^{13} \mathrm{C}$ NMR $\left(125 \mathrm{MHz}, \mathrm{CDCl}_{3}\right): \delta 168.1,167.2,165.4,158.6,134.3,131.4,127.2,113.9$, $60.9,55.2,51.6,37.0,28.6,28.4,27.5,26.2,14.1$.

HRMS ( $\left(\mathrm{M}^{+}\right)$calcd for $\mathrm{C}_{24} \mathrm{H}_{36} \mathrm{~N}_{2} \mathrm{O}_{5} 432.2624$, found 432.2629. 
${ }^{1}$ H-NMR (Compound 39)

isems

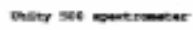

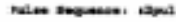

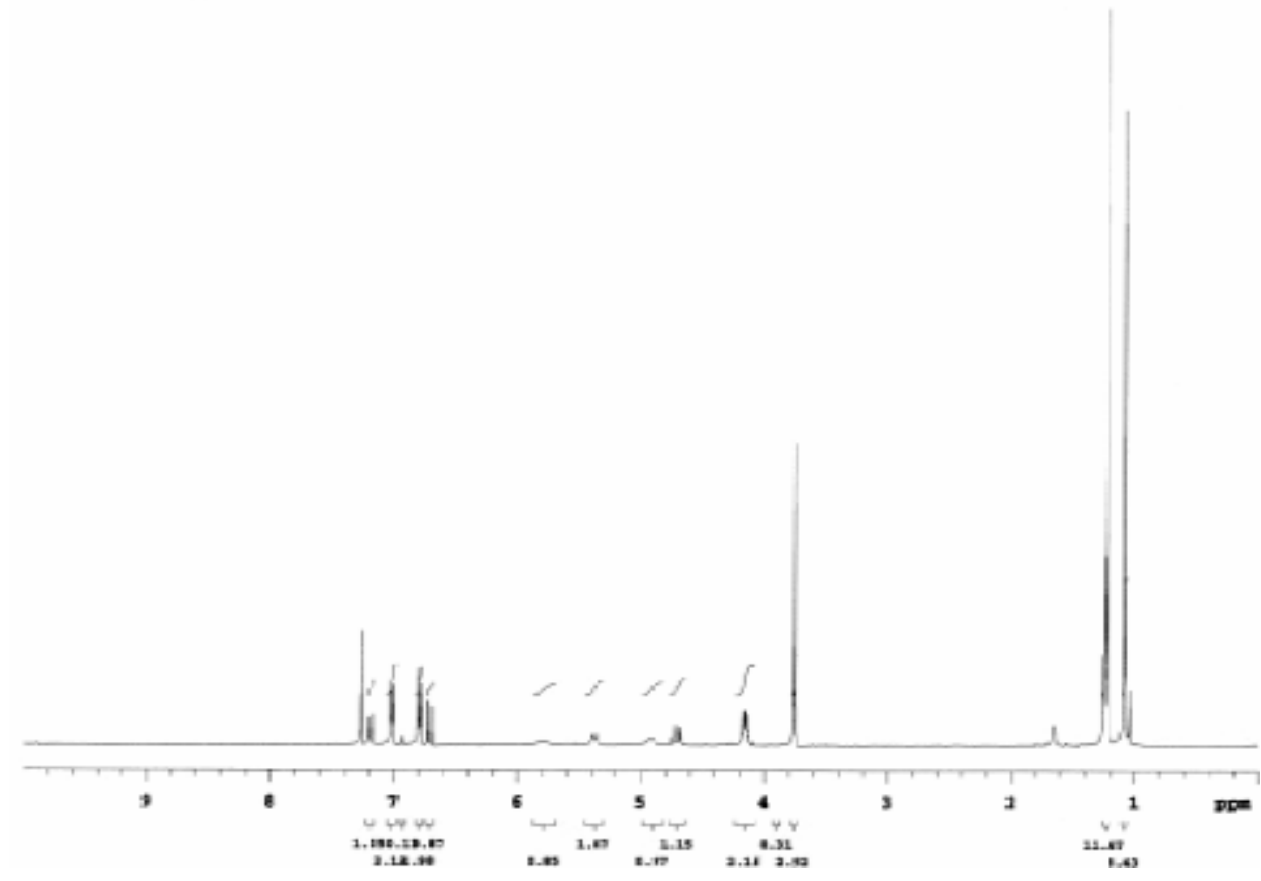

${ }^{13}$ C-NMR (Compound 39)

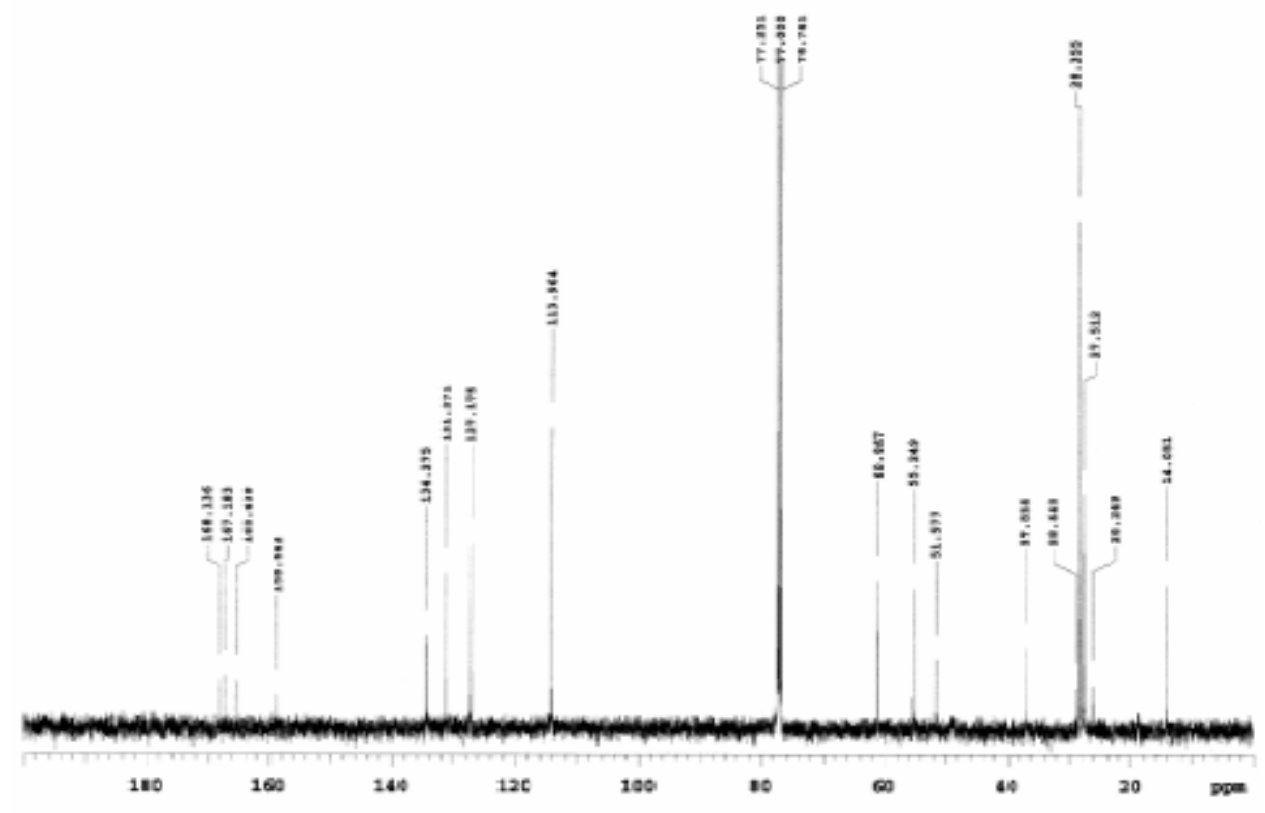


(E)-ethyl 3-(N-(1-(tert-butylcarbamoyl)-2-methylpropyl)-N-(3,4-dimethoxybenzyl)carbamoyl)acrylate (40)<smiles>CCOC(=O)C=CC(=O)N(Cc1ccc(OC)c(OC)c1)C(C)(C(=O)NC(C)(C)C)C(C)(C)C</smiles>

40

${ }^{1} \mathrm{H}$ NMR $\left(500 \mathrm{MHz}, \mathrm{CDCl}_{3}\right): \delta$ 7.24-7.32 (d, 1H, J=15.2 Hz, $\left.-\mathrm{CH}=\mathrm{CH}-\right)$, 6.66-6.82 (m, $4 \mathrm{H}$, aryl overlap with $-\mathrm{CH}=\mathrm{CH}-), 4.73(\mathrm{~d}, 1 \mathrm{H}, J=16.7 \mathrm{~Hz}, \mathrm{Bn}), 4.61(\mathrm{~d}, 1 \mathrm{H}, J=17.2$ $\mathrm{Hz}, \mathrm{Bn}), 4.27$ (bs, 1H, $-\mathrm{N} \underline{\mathrm{H}}$ ), 4.23 (bs, 1H, $\left.\mathrm{H}_{\mathrm{a}}\right), 4.12-4.21\left(\mathrm{~m}, 2 \mathrm{H},-\mathrm{OC}_{2} \mathrm{CH}_{3}\right), 3.81$ (s, $\left.6 \mathrm{H},-\mathrm{OC}_{3}\right), 2.32-2.45\left(\mathrm{~m}, 1 \mathrm{H}, \mathrm{H}_{\mathrm{b}}\right), 1.21-1.27\left(\mathrm{~m}, 12 \mathrm{H}, t-\mathrm{Bu}\right.$ overlap with $\left.-\mathrm{OCH}_{2} \mathrm{CH}_{3}\right)$, 0.93 (d, 3H, $J=6.6 \mathrm{~Hz}, i-\operatorname{Pr}), 0.78$ (d, 3H, $J=6.6 \mathrm{~Hz}, i-\operatorname{Pr})$.

${ }^{13} \mathrm{C}$ NMR $\left(125 \mathrm{MHz}, \mathrm{CDCl}_{3}\right): \delta 168.9,166.6,165.3,148.9,148.4,134.1,131.9,129.5$, $119.1,111.0,110.2,61.1,55.8,51.2,28.5,27.4,19.5,19.0,14.0$.

$\operatorname{HRMS}\left(\mathrm{M}^{+}\right)$calcd for $\mathrm{C}_{24} \mathrm{H}_{36} \mathrm{~N}_{2} \mathrm{O}_{6} 448.2573$, found 448.2590

\section{$\underline{{ }^{1} \text { H-NMR (Compound 40) }}$}
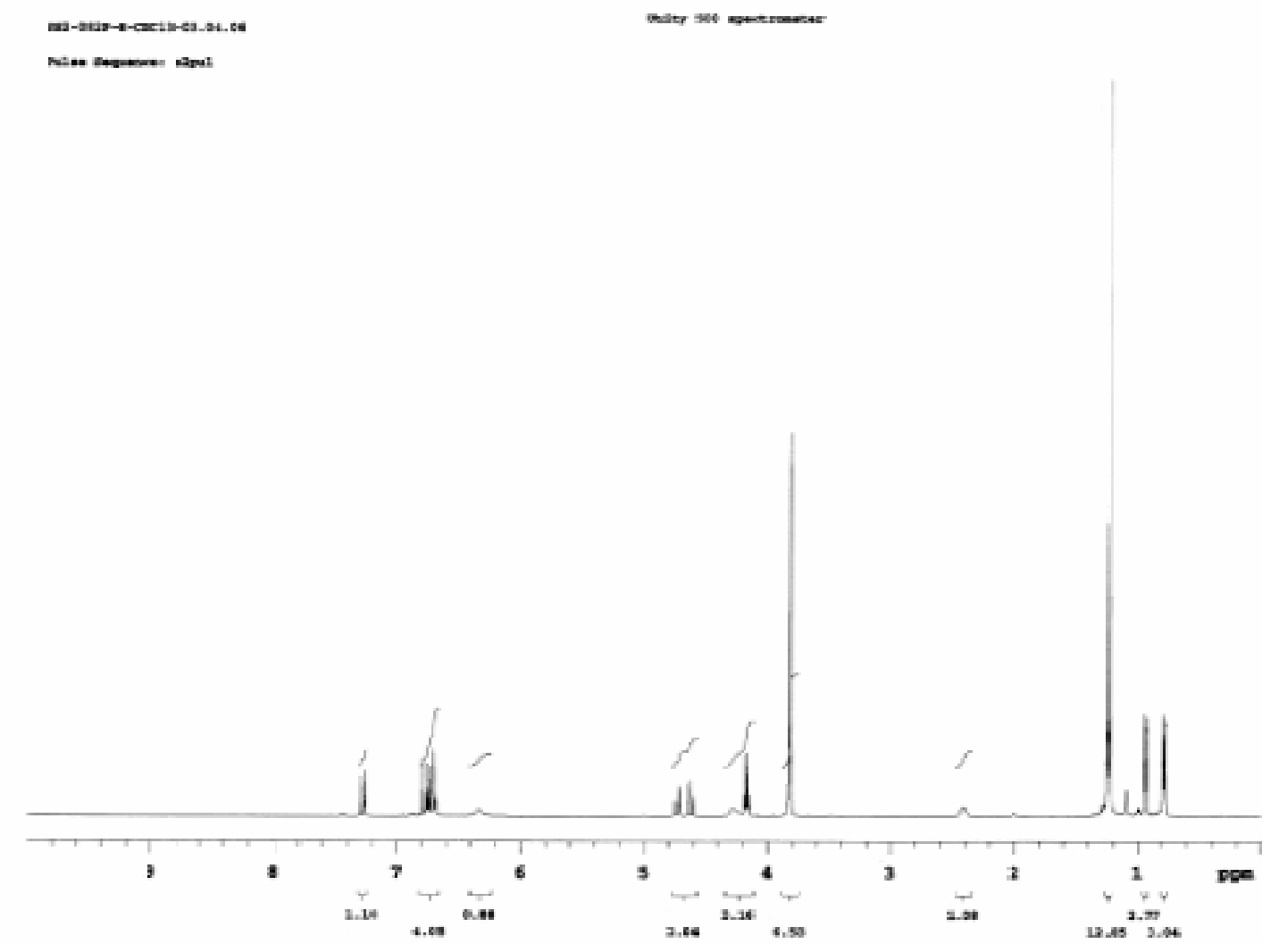


\section{${ }^{13}$ C-NMR (Compound 40)}

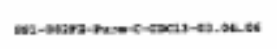

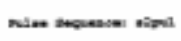

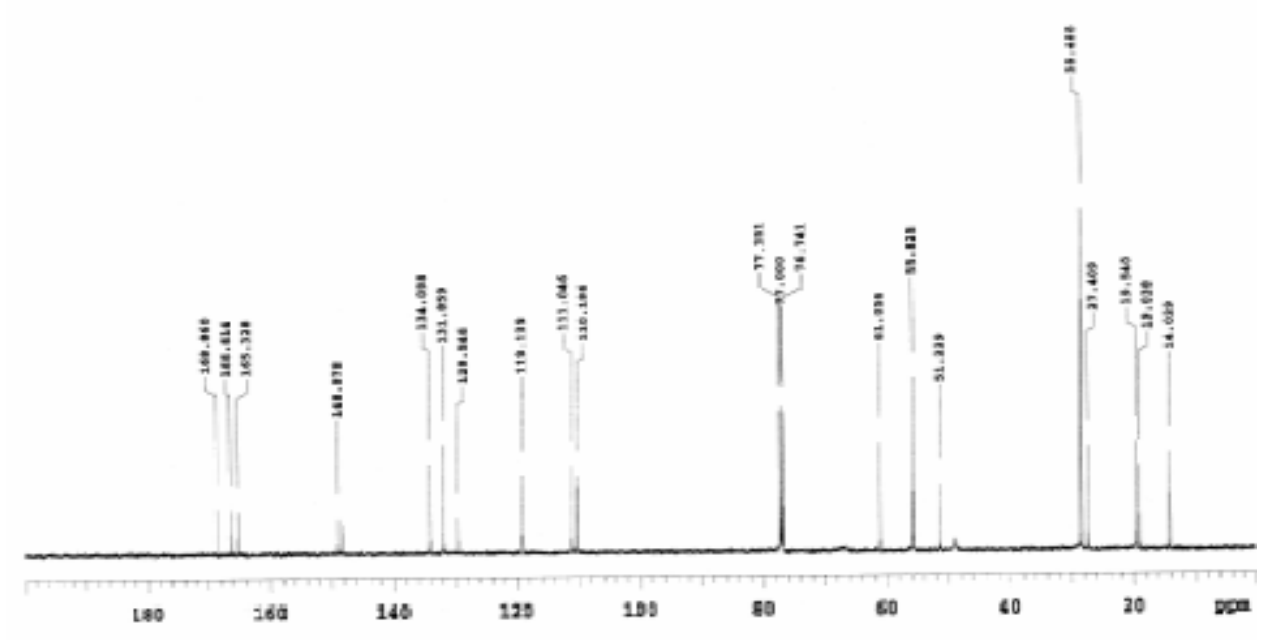

(E)-ethyl 3-(N-(1-(tert-butylcarbamoyl)-2-methylpropyl)-N-benzylcarbamoyl)acrylate (41)

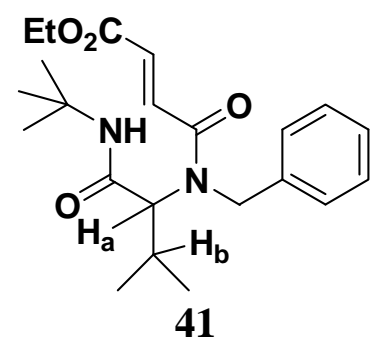

${ }^{1} \mathrm{H}$ NMR (500 MHz, $\left.\mathrm{CDCl}_{3}\right): \delta$ 7.09-7.26 (m, 6H, aryl overlap with $-\mathrm{C} \underline{\mathrm{H}}=\mathrm{CH}-$ ), $6.74(\mathrm{~d}$, $1 \mathrm{H}, J=15.7 \mathrm{~Hz},-\mathrm{CH}=\mathrm{C} \underline{\mathrm{H}}-), 6.37(\mathrm{bs}, 1 \mathrm{H},-\mathrm{NH}), 4.84(\mathrm{~d}, 1 \mathrm{H}, J=17.8 \mathrm{~Hz}, \mathrm{Bn}), 4.64$ (d, $1 \mathrm{H}, J=17.2 \mathrm{~Hz}, \mathrm{Bn}), 4.4\left(\mathrm{~d}, 1 \mathrm{H}, J=8.6 \mathrm{~Hz}, \mathrm{H}_{\mathrm{a}}\right), 4.08-4.16\left(\mathrm{~m}, 2 \mathrm{H},-\mathrm{OC}_{2} \mathrm{CH}_{3}\right), 2.31-$ $2.42\left(\mathrm{~m}, 1 \mathrm{H}, \mathrm{H}_{\mathrm{b}}\right), 1.21(\mathrm{~s}, 9 \mathrm{H}, t-\mathrm{Bu}), 1.19\left(\mathrm{t}, 3 \mathrm{H}, J=7.1 \mathrm{~Hz},-\mathrm{OCH}_{2} \underline{\mathrm{C}}_{3}\right), 0.92(\mathrm{~d}, 3 \mathrm{H}, J$ $=6.6 \mathrm{~Hz}, i-\operatorname{Pr}), 0.78(\mathrm{~d}, 3 \mathrm{H}, J=6.6 \mathrm{~Hz}, i-\operatorname{Pr})$.

${ }^{13} \mathrm{C} \mathrm{NMR}\left(125 \mathrm{MHz}, \mathrm{CDCl}_{3}\right): \delta 168.5,166.5,165.1,137.2,133.9,131.9,128.5,127.3$, $126.5,60.9,51.2,28.3,27.4,19.4,18.7,13.9$.

HRMS $\left(\mathrm{M}^{+}\right)$calcd for $\mathrm{C}_{22} \mathrm{H}_{32} \mathrm{~N}_{2} \mathrm{O}_{4} 388.2362$, found 388.2365. 


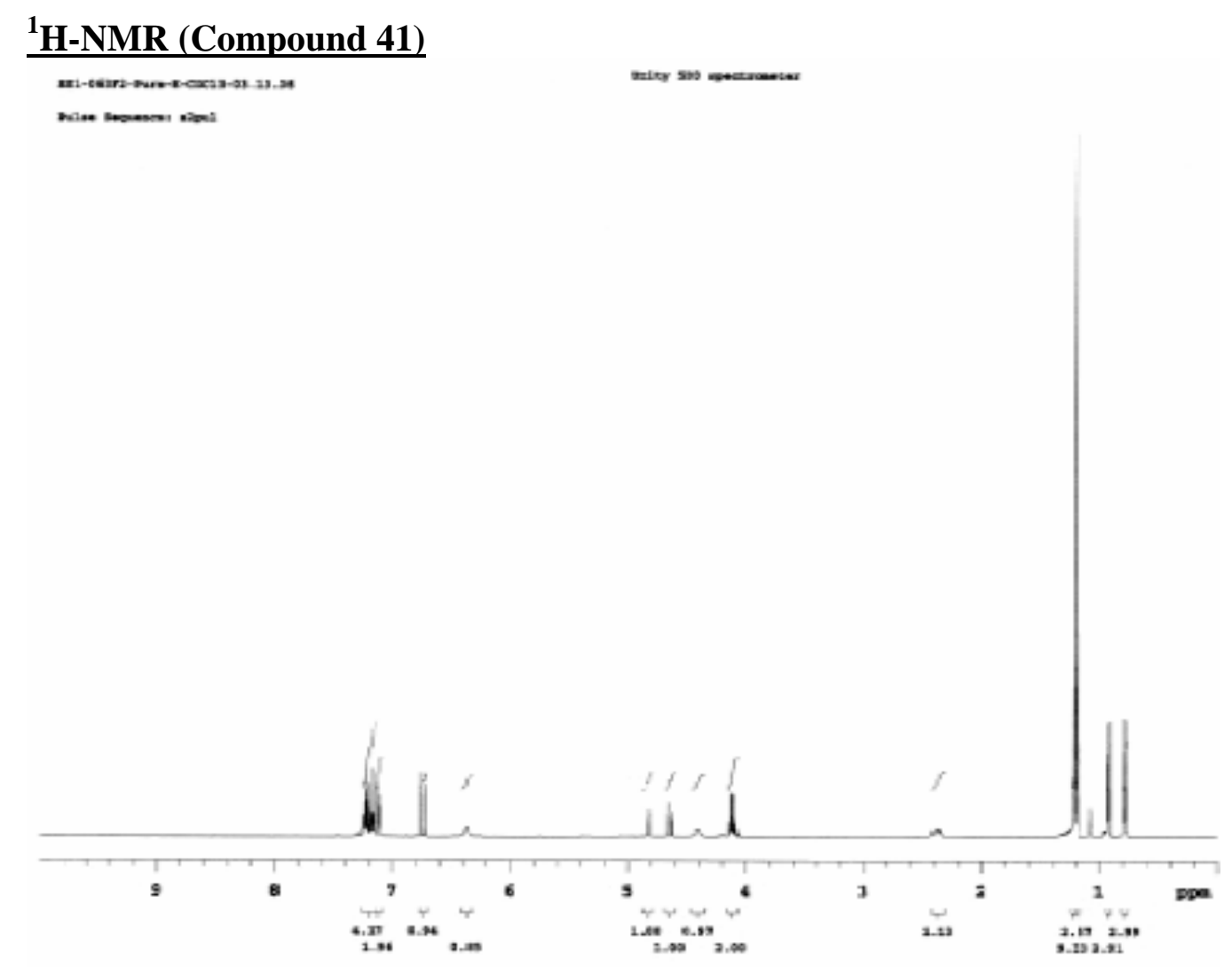

${ }^{13}$ C-NMR (Compound 41) 

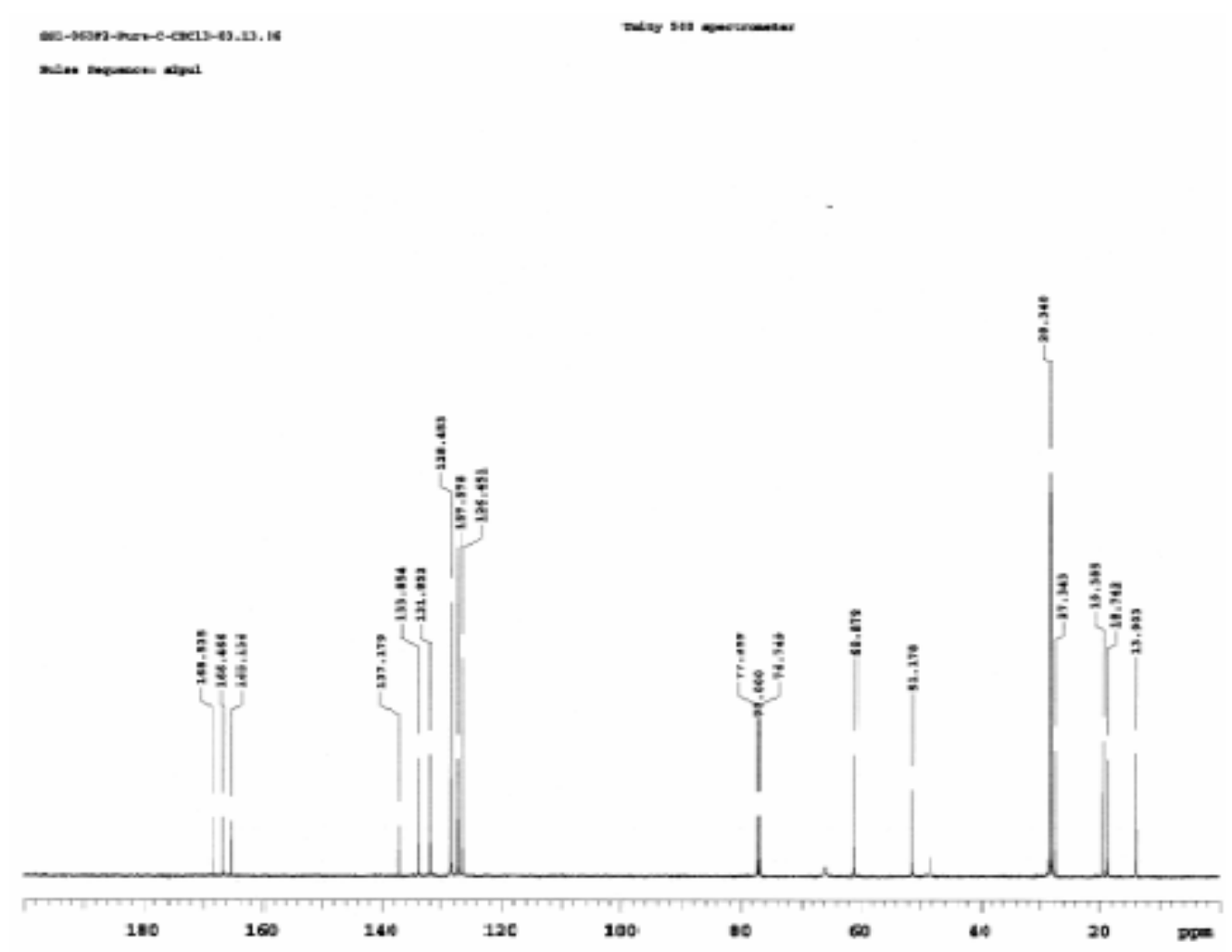

\section{Acyclic Ugi Product for entries 10/18 and 11/19:}

(E)-Ethyl 3-(N-((benzylcarbamoyl)(thiophen-2-yl)methyl)-N-(3,4-dimethoxybenzyl)carbamoyl)acrylate (SI-A)

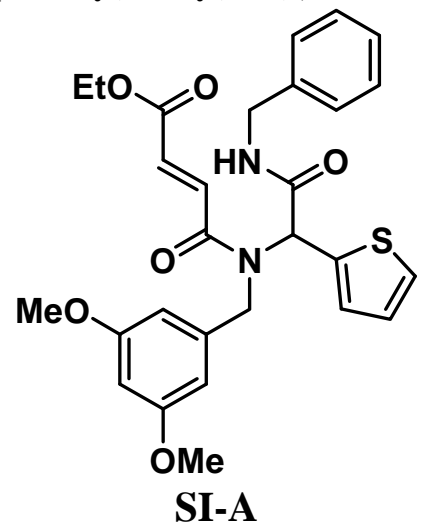

${ }^{1} \mathrm{H}$ NMR $\left(500 \mathrm{MHz}, \mathrm{CDCl}_{3}\right): \delta$ 7.17-7.34 (m, 6H, aryl), $7.12(\mathrm{~m}, 1 \mathrm{H}, \operatorname{aryl}), 6.90-6.95(\mathrm{~m}$, $1 \mathrm{H}$, aryl $), 6.87(\mathrm{~d}, 1 \mathrm{H}, J=14.7 \mathrm{~Hz}, \mathrm{HC}=\mathrm{CH}), 6.23-6.33(\mathrm{~m}, 4 \mathrm{H}), 5.96(\mathrm{~s}, 1 \mathrm{H}), 4.71(\mathrm{~s}$, $2 \mathrm{H}), 4.52(\mathrm{dd}, 1 \mathrm{H}, J=5.5,14.9 \mathrm{~Hz}, \mathrm{Bn}), 4.40(\mathrm{dd}, 1 \mathrm{H}, J=5.6,15.2 \mathrm{~Hz}, \mathrm{Bn}), 4.19$ (q, 2H, $\left.J=7.1 \mathrm{~Hz},-\mathrm{OCH}_{2} \mathrm{CH}_{3}\right), 3.7\left(\mathrm{~s}, 6 \mathrm{H}, \mathrm{OCH}_{3}\right), 1.26\left(\mathrm{t}, 3 \mathrm{H}, J=7.1 \mathrm{~Hz}, \mathrm{CH}_{3}\right)$.

${ }^{13} \mathrm{C}$ NMR $\left(125 \mathrm{MHz}, \mathrm{CDCl}_{3}\right): \delta 167.9,166.1,165.2,161.0,138.9,137.7,135.9,133.4$, $132.8,130.1,128.7,128.2$, 127.6, 127.5, 126.7, 104.3, 99.8, 61.1, 59.1, 55.3, 50.8, 43.8, 14. 
HRMS: EIMS ( $\left(\mathrm{M}^{+}\right)$calcd for $\mathrm{C}_{28} \mathrm{H}_{30} \mathrm{~N}_{2} \mathrm{O}_{6} \mathrm{~S}$ 522.1825, found 522.1816.

${ }^{1}$ H-NMR (Compound SI-A)
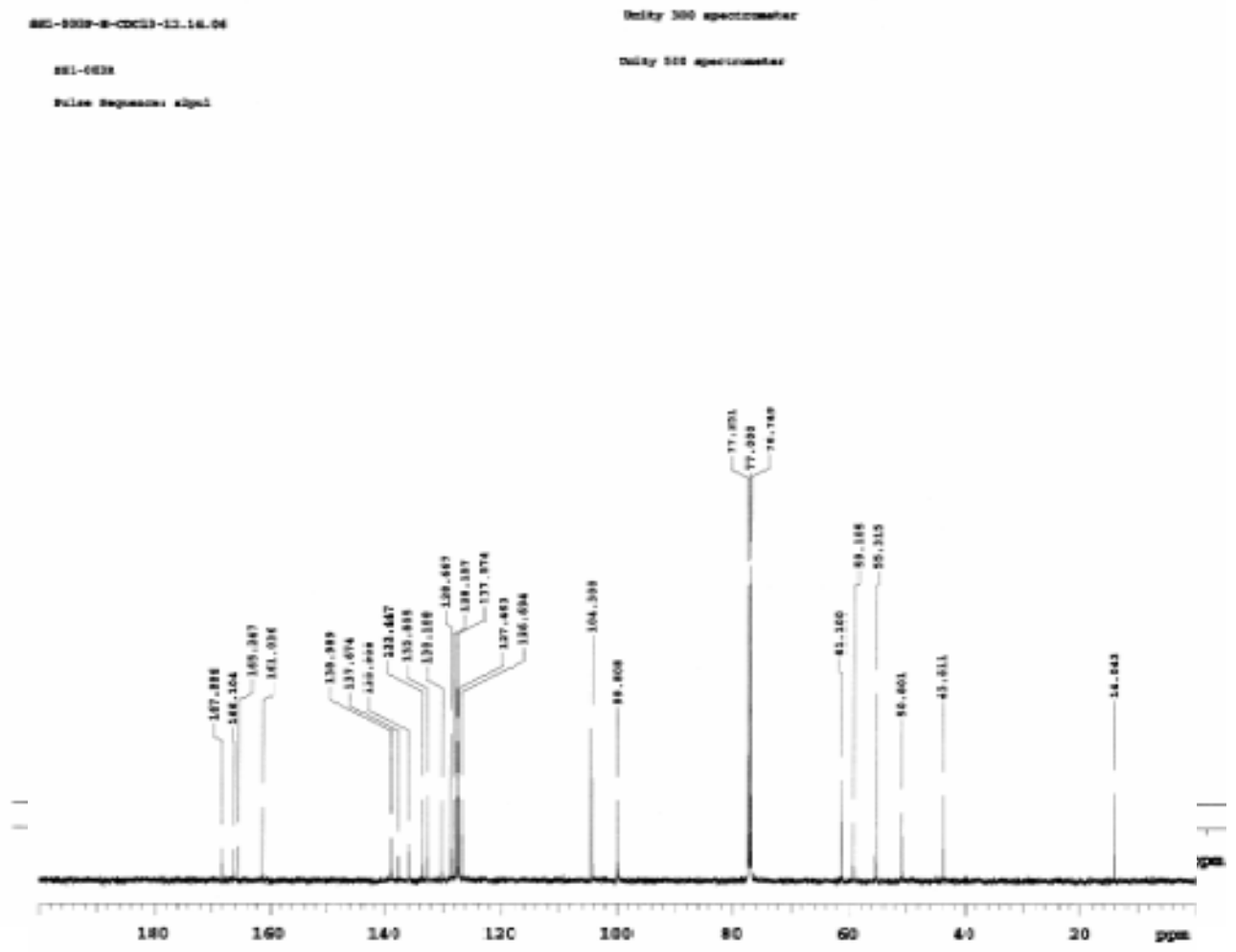

${ }^{13}$ C-NMR (Compound SI-A)

(E)-ethyl 4-((2-(benzylamino)-2-oxo-1-(thiophen-2-yl)ethyl)(4-methoxybenzyl)amino)-4-oxobut-2-enoate (SI-B)<smiles>[R][SiH2]c1ccc(CN(C(=O)/C=C/C(=O)OCC)C(C)(C(=O)NCc2ccccc2)c2cccs2)cc1</smiles>

${ }^{1} \mathrm{H}$ NMR $\left(500 \mathrm{MHz}, \mathrm{CDCl}_{3}\right): \delta$ 7.19-7.31 (m, 7H, aryl and $-\mathrm{CH}=\mathrm{CH}-$ overlap), $7.08(\mathrm{~d}$, $1 \mathrm{H}, J=3.1 \mathrm{~Hz}$, aryl $), 6.98(\mathrm{~d}, 2 \mathrm{H}, J=9.1 \mathrm{~Hz}$, aryl), $6.89(\mathrm{dd}, 1 \mathrm{H}, J=3.6,5.1 \mathrm{~Hz}$, aryl), $6.78(\mathrm{~d}, 1 \mathrm{H}, J=15.2 \mathrm{~Hz},-\mathrm{CH}=\mathrm{CH}-), 6.69-6.76\left(\mathrm{~m}, 3 \mathrm{H}\right.$, aryl), $6.09\left(\mathrm{~s}, 1 \mathrm{H}, \mathrm{H}_{\mathrm{a}}\right), 4.71(\mathrm{dd}$, $2 \mathrm{H}, J=17.2,21.3, \mathrm{Bn}), 4.47$ (dd, 1H, $J=6.1,14.8 \mathrm{~Hz}, \mathrm{Bn}), 4.35$ (dd, 1H, $J=14.8 \mathrm{~Hz}$, $\mathrm{Bn}$ ), $\left.4.169 \mathrm{q}, 2 \mathrm{H}, J=7.3 \mathrm{~Hz},-\mathrm{OC}_{2} \mathrm{CH}_{3}\right), 3.55$ (s, 3H, $\left.-\mathrm{OC}_{3}\right), 1.25$ (t, $3 \mathrm{H}, J=7.3 \mathrm{~Hz}$, $\left.\mathrm{OCH}_{2} \underline{\mathrm{CH}}_{3}\right)$, 
${ }^{13} \mathrm{C}$ NMR $\left(125 \mathrm{MHz}, \mathrm{CDCl}_{3}\right): \delta 168.1,165.9,165.1,158.8,137.7,135.9,133.6,132.3$, $129.7,128.5,128.4,127.7,127.6,127.4,127.2$, 126.5, 113.9, 60.9, 58.4, 55.1, 49.8, 43.5, 13.9.

\section{$\underline{{ }^{1} \text { H-NMR (Compound SI-B) }}$}

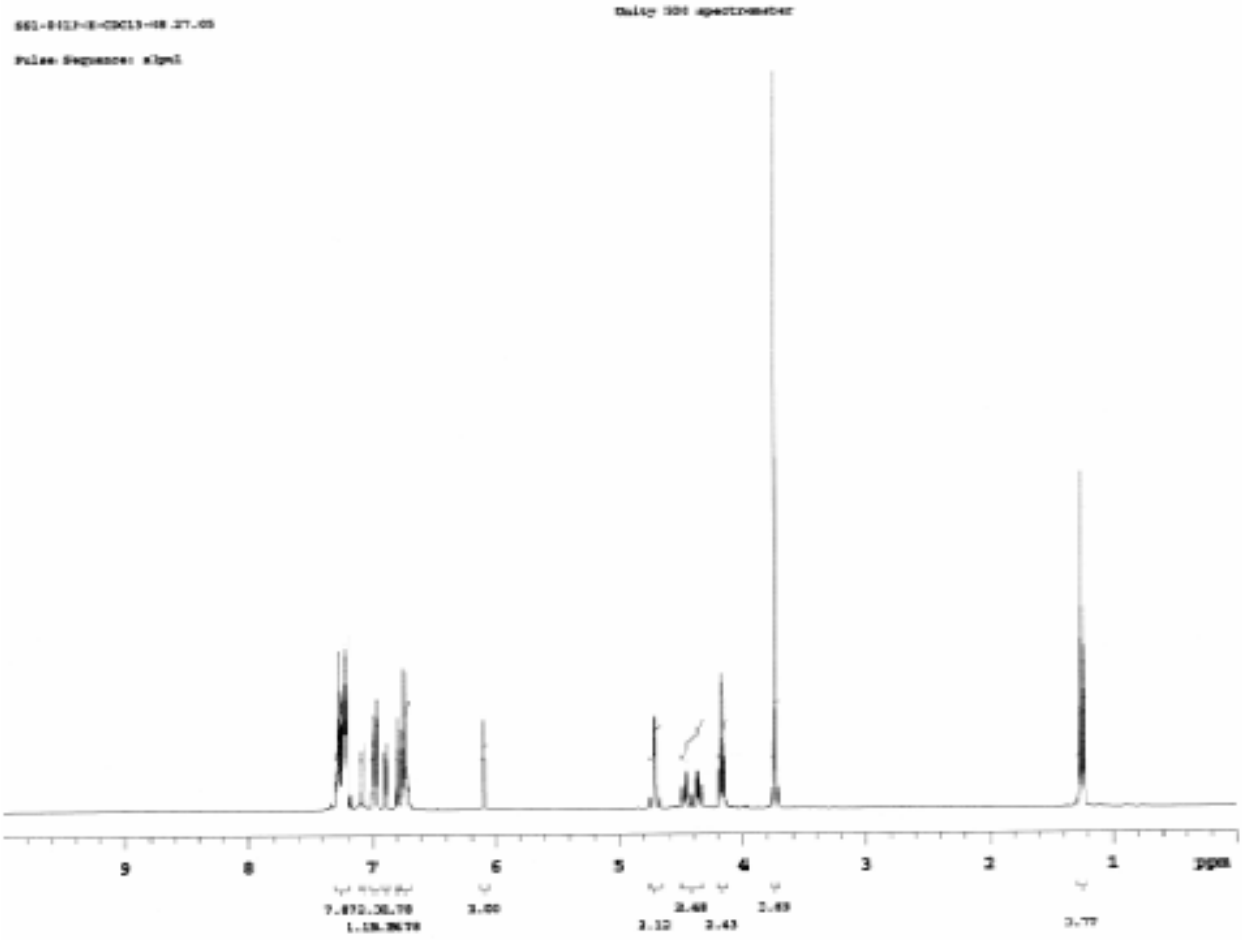

\section{${ }^{13}$ C-NMR (Compound SI-B)}

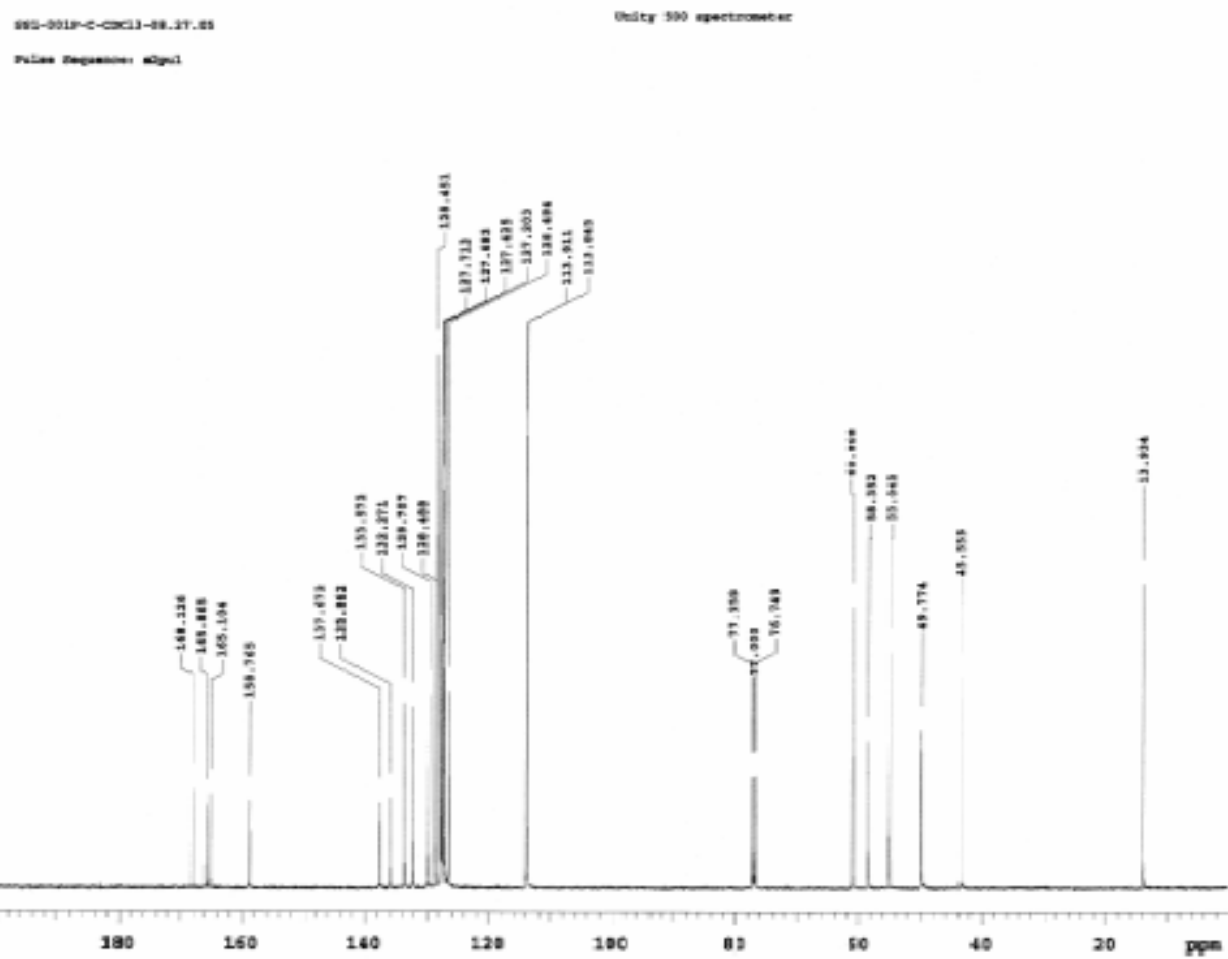

